\title{
CALIFORNIA INSTITUTE OF TECHNOLOGY
}

EARTHQUAKE ENGINEERING RESEARCH LABORATORY

\section{A BAYESIAN PROBABILISTIC APPROACH TO STRUCTURAL HEALTH MONITORING}

\author{
BY
}

MichAEL W. VANIK

REPORT NO. EERL 97-07

PASADENA, CALIFORNIA 
A REPORT ON RESEARCH PARTIALLY SUPPORTED BY THE EARTHQUAKE RESEARCH AfFILIATES PROGRAM OF CALTECH UNDER THE SUPERVISION OF JAMES L. BECK 


\title{
A Bayesian Probabilistic Approach to Structural Health Monitoring
}

\author{
Thesis by \\ Michael W. Vanik
}

In Partial Fulfillment of the Requirements

for the Degree of

Doctor of Philosophy

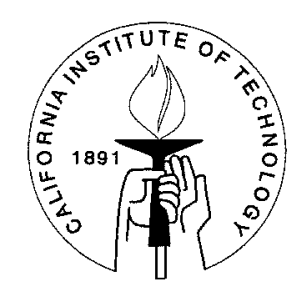

California Institute of Technology

Pasadena, California 
(C) 1997

Michael W. Vanik

All rights Reserved 


\section{Acknowledgements}

When I wandered, like a lost puppy, over from the Aeronautics Department looking for a new home and a new direction, Dr. James L. Beck took me into his group. I thank him for his support and encouragement over the last four and a half years. Without his input and advice, this thesis could not have come into being.

I would also like to thank my first advisor at Caltech, Dr. Wolfgang G. Knauss. His was the first voice I heard from Caltech. Although we parted ways, I would not have come to California if not for his efforts.

My friends have been invaluable during my time here. My group of officemates in 110 Thomas, Anders Carlson, Eduardo Chan, Bill Goodwine, Ayhan Irfanoglu, Scott May and Dave Polidori, have all helped keep me sane during the last five years. To Raff D'Andrea, Dave Geraghty, John Latz, Will Law, and Mike Tierney I am grateful for many lasting memories. Todd Fernandez, Cathy Moore and Scout have reminded me that there is life outside of and after Caltech. Bob Uy has offered an ear for my griping on many topics and been excellent company on Thursday nights. To the few I mention here and the many I am certain I have forgotten, I offer my thanks.

My parents, mom, dad, ma, and pop, played no small role in my getting to this point. I appreciate their support and love through all my years schooling.

Finally, I want to thank my wife and dedicate this thesis to her. She is my best friend, my never ending source of comfort, and my only love. Without her help, constant understanding, and inspiration I could not have completed this thesis. She makes me better than I am and I love her. 


\title{
A Bayesian Probabilistic Approach to Structural Health Monitoring
}

by

\author{
Michael W. Vanik
}

\author{
In Partial Fulfillment of the \\ Requirements for the Degree of \\ Doctor of Philosophy
}

\section{Abstract}

A Bayesian probabilistic methodology for on-line structural health monitoring which addresses the issue of parameter uncertainty inherent in problem is presented. The method uses modal parameters for a limited number of modes identified from measurements taken at a restricted number of degrees of freedom of a structure as the measured structural data. The application presented uses a linear structural model whose stiffness matrix is parameterized to develop a class of possible models. Within the Bayesian framework, a joint probability density function (PDF) for the model stiffness parameters given the measured modal data is determined. Using this PDF, the marginal PDF of the stiffness parameter for each substructure given the data can be calculated.

Monitoring the health of a structure using these marginal PDFs involves two steps. First, the marginal PDF for each model parameter given modal data from the undamaged structure is found. The structure is then periodically monitored and updated marginal PDFs are determined. A measure of the difference between the calibrated and current marginal PDFs is used as 
a means to characterize the health of the structure. A procedure for interpreting the measure for use by an expert system in on-line monitoring is also introduced.

The probabilistic framework is developed in order to address the model parameter uncertainty issue inherent in the health monitoring problem. To illustrate this issue, consider a very simplified deterministic structural health monitoring method. In such an approach, the model parameters which minimize an error measure between the measured and model modal values would be used as the "best" model of the structure. Changes between the model parameters identified using modal data from the undamaged structure and subsequent modal data would be used to find the existence, location and degree of damage. Due to measurement noise, limited modal information, and model error, the "best" model parameters might vary from one modal dataset to the next without any damage present in the structure. Thus, difficulties would arise in separating normal variations in the identified model parameters based on limitations of the identification method and variations due to true change in the structure. The Bayesian framework described in this work provides a means to handle this parametric uncertainty.

The probabilistic health monitoring method is applied to simulated data and laboratory data. The results of these tests are presented. 


\section{Contents}

1 Introduction 1

1.1 A Need For Structural Health Monitoring . . . . . . . . . . 1

1.2 Structural Health Monitoring ............. 3

1.2.1 Local Structural Health Monitoring . . . . . . . . . 4

1.2.2 Global Structural Health Monitoring ........ 5

1.2.3 Local and Global Method Working Together . . . . . 12

1.3 What Does This Work Present? . . . . . . . . . . . 13

2 Theoretical Development 17

2.1 Introduction ..................... 17

2.2 Bayesian Probability ................ 18

2.3 Outline of Bayesian SHM . . . . . . . . . . . . 20

2.4 Formulating the Updated PDF . . . . . . . . . . . 23

2.4 .1 Measured Data ............... 23

2.4 .2 Structural Model Class . . . . . . . . . . . 26

2.4.3 Probability Model Class . . . . . . . . . . . 33

2.4.4 Updated PDF for the Model Parameters . . . . . . 41

2.5 Bayesian SHM Framework . . . . . . . . . . . 55

2.5.1 The Undamaged and Potentially Damaged PDFs . . . 56

2.5.2 Observations on the Marginal Distributions . . . . 60

2.5.3 Defining the Probability of Variation . . . . . . . 62 
2.5.4 Considerations in the Use of $P^{v a r} \ldots \ldots \ldots \ldots$

2.5 .5 Sounding an Alarm . . . . . . . . . . . . . 67

2.5 .6 Applying $P^{v a r}$ for SHM $\ldots \ldots \ldots \ldots 68$

2.5.7 Degree of Damage . . . . . . . . . . . . . 70

2.5.8 Calculating the Cumulative Distributions . . . . . . 71

2.6 Summary of the SHM Method . . . . . . . . . . . . . 75

3 Simulated Data Testing $\quad 81$

3.1 Introduction . . . . . . . . . . . . . . . . . 81

3.1.1 Common Definitions . . . . . . . . . . . . . 82

3.1.2 Brief Descriptions of the Examples . . . . . . . . 85

3.1 .3 Picturing Damage . . . . . . . . . . . . . . . . . 85

3.2 Two DOF Shear Structure . . . . . . . . . . . . . . 87

3.2 .1 Results of the Testing . . . . . . . . . . . . 90

3.2.2 Observations on the 2-DOF Example . . . . . . . . 111

3.3 Ten DOF Shear Structure . . . . . . . . . . . . . . . 113

3.3.1 Initialization Phase . . . . . . . . . . . . . 116

3.3.2 Monitoring Phase . . . . . . . . . . . . . . . . . 117

3.3.3 Concluding Remarks on the 10-DOF Example . . . . . 123

4 Conclusions and Future Work $\quad 124$

4.1 Future Work . . . . . . . . . . . . . . . . . . . . . . . . . 124

4.1 .1 Additional Testing . . . . . . . . . . . . . . . 124

4.1.2 Improving the Method . . . . . . . . . . . . . 126

4.1 .3 Automation . . . . . . . . . . . . . . . 128

4.2 Limitations of The SHM Method . . . . . . . . . . 128

4.3 Conclusions . . . . . . . . . . . . . . . . . 131

A Probability Model Details 134 
$\begin{array}{ll}\text { B Calculating the Minimum and Hessian } & 140\end{array}$

B.1 Preliminary Development. . . . . . . . . . . . . . . . 140

B.1.1 Alternate Forms of the MMOF . . . . . . . . . 141

B.1.2 Derivatives of $J(\theta, \alpha) \ldots \ldots \ldots \ldots \ldots$

B.1.3 Some Final Notation . . . . . . . . . . . . . . . . . 144

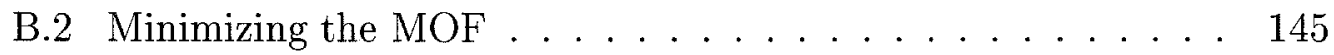

B.3 Derive the Hessian . . . . . . . . . . . . . . . . 149 


\section{List of Figures}

2.1 Two Degree of Freedom Shear Structure Model . . . . . . . . 32

2.2 Updated PDF with Non-informative Initial PDF on $\alpha \ldots \ldots 7$

2.3 Updated PDF with Informative Initial PDF on $\alpha \ldots 48$

2.4 General PDFs derived from different data sets: $\tilde{p}_{k}^{p d}$ left . . . . 61

2.5 General PDFs derived from different data sets: $\tilde{p}_{k}^{p d}$ right . . 61

2.6 Depiction of $\theta_{k i}^{p d}<\theta_{i}^{u d} \ldots \ldots \ldots \ldots 4$

3.1 Possible Initial Distributions for $\theta$. . . . . . . . . . . . 84

3.2 Typical probability of variation plot for a given monitoring time 86

3.3 Two Degree of Freedom Shear Structure Model . . . . . . . . 87

3.4 Most probable model parameters versus number of undamged modal data set: Test Case $1 \ldots$. . . . . . . . . . . 91

3.5 Most probable model parameter versus number of undamged modal data set: Test Case $2 \ldots . . \ldots 92$

3.6 Most probable model parameters versus number of undamged

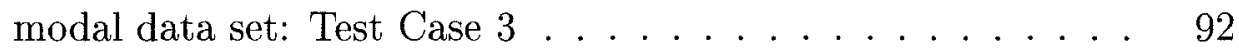

3.7 Most probable model parameters versus number of undamged modal data set: Test Case $1 \ldots . . . . . . . . . . .993$

3.8 Sensitivities of the modal frequencies with variations in the model parameters . . . . . . . . . . . . . . . 94

3.9 Alarm functions for different value of $\gamma$ : Case $1 \ldots 96$

3.10 Alarm functions for different values of $\gamma$ : Case $2 \ldots$. . . . . . 96 
3.11 Alarm functions for different values of $\gamma$ : Case $4 \ldots$. . . . . 96

$3.12 P^{v a r}$ over sixteen monitoring cycles: No damage . . . . . . . . 98

3.13 Relative frequency of false alarms: Case 1, All $k \ldots 99$

3.14 Relative frequency of false alarms: Case 2 , All $k$. . . . . 100

3.15 Relative frequency of false alarms: Case 4, All $k$. . . . . . 100

3.16 Relative frequency of false alarms: Case $1, k=1 \ldots$. . . . . 100

3.17 Relative frequency of false alarms: Case $2, k=1$. . . . . . 101

3.18 Relative frequency of false alarms: Case 4 . . . . . . . . . . . 101

$3.19 P^{v a r}$ over sixteen monitoring cycles: $2 \%$ damage . . . . . . . 108

$3.20 P^{v a r}$ over eight monitoring cycles: $5 \%$ damage . . . . . . . . . 109

$3.21 P^{v a r}$ over eight monitoring cycles: $10 \%$ damage . . . . . . . 110

3.22 Alarm functions for four parameters in the 10-DOF example . 117

$3.23 P^{v a r}$ over eight monitoring cycles: Test Case 1, Damage Case

D2, Fifth Story Substructure Parameter . . . . . . . . 122 


\section{List of Tables}

1.1 SHM Problem Statement . . . . . . . . . . . . . . . 14

3.1 Definition of noise levels on the modal parameters . . . . . . 82

3.2 Fundamental Model Parameters for 2-DOF Shear Structure . 88

3.3 Fundamental Modal Parameters for 2-DOF Shear Structure . 89

3.4 Cases considered in stabilizing the undamaged PDF for the 2DOF shear model . . . . . . . . . . . . . 91

3.5 Damage cases for the 2-DOF example . . . . . . . . . . . 102

3.6 Results of 2-DOF damage analysis: Case 1 . . . . . . . . 104

3.7 Results of 2-DOF damage analysis: Case 2 . . . . . . . . 105

3.8 Results of 2-DOF damage analysis: Case $4 \ldots 106$

3.9 Fundamental Model Parameters for 10-DOF Shear Structure . 114

3.10 Substructuring Options for the 10-DOF Example . . . . . . 114

3.11 Different test cases considered in 10-DOF example . . . . 115

3.12 Damage cases for the 10-DOF example . . . . . . . . . . 116

3.13 Results of 10-DOF damage analysis: Case 1 . . . . . . . 118

3.14 Results of 10-DOF damage analysis: Case 2 . . . . . . . . . 119

3.15 Results of 10-DOF damage analysis: Case 3 . . . . . . . . . . 120

3.16 Results of 10-DOF damage analysis: Case 4 . . . . . . . . 120 


\section{Chapter 1}

\section{Introduction}

\section{$1.1 \quad$ A Need For Structural Health Monitoring}

Shortly after 1:00 A.M. on June 28, 1983, a suspended span of the Mianus river bridge on Interstate 95 in Connecticut fell to the water below. Several cars and trucks followed the span into the river, leading to three deaths and three injuries (Levy and Salvadori 1992). A larger tragedy on this highly traveled roadway was avoided only because of the early hour of the event. The failure of the bridge was due in part to the corrosion of steel support members and cyclic loading of the traffic. The key structural failure which is suspected to have lead to the collapse may have occurred, "hours or even days" before the span actually broke away. Only nine months earlier, the bridge had been inspected by the Connecticut Department of Transportation.

During the January 17, 1994 Northridge earthquake in southern California, many structures were shaken and severely damaged. Over 100,000 buildings were visually inspected and "tagged" with safety levels roughly according to the guidelines set forth in ATC 20-Procedures for Postearthquake Safety Evaluation of Buildings (Applied Technology Council 1989). The inspections left most of the steel moment-frame structures green tagged (i.e. safe for occupancy). In the weeks which followed the event, discoveries were made that 
many of these steel moment-frame structures suffered significant joint damage during the earthquake (EERI 1996). These situations were especially difficult to diagnose as a result of the steel generally being encased in fireproofing, non-structural cladding, and other deterrents to inspection. The reports of problems with the steel framed structures grew as anomalies in structures were discovered. In one particular structure, the building was found to be leaning to the north after occupants kept complaining about operational problems with the elevator. As of this writing, more than 100 steel frame structures have been found to have steel connections which failed as a result of shaking from the Northridge earthquake.

On April 28,1988, an Aloha Airlines Boeing 737 suddenly lost an entire fuselage section while at 24,000 feet (Ott and O'lone 1988). Sixty-nine passengers were injured and one flight attendant was swept from the plane. Inspection of the craft in the aftermath of the accident revealed significant cracking in the fuselage. The suspected cause of the cracks was fatigue. Within the six months prior to the accident, the plane had been inspected and undergone some minor repair work in accordance with the then current FAA and Boeing guidelines.

These are a small sampling of the available examples wherein structural failure or potential structural failure arose due to loss of structural integrity. They also share a commonality in that all are cases where existing inspection regulations and techniques were insufficient to recognize the possible danger in a timely and detailed manner. For example, at the time of the Mianus River Bridge collapse, the state of Connecticut used six two-man teams to biennially inspect over thirty-five hundred bridges in Connecticut. Each team surveyed nearly three hundred bridges a year. This created a dangerous situation in which the inspection interval was long, and the level of inspection was limited to simple observations. Thus, the potential for structural failure of the Mianus 
River Bridge was missed.

A tool which is able to monitor the state of a structure in a detailed manner and on a more acceptable time scale and warn of potential problems would improve structural safety. Establishing the likely location and nature of structural weakness would also be an invaluable capability. Such information would warn inspectors if the structure may be damaged, where to look for the loss of integrity, and what type of loss to expect. Thus enters the concept of structural health monitoring.

\subsection{Structural Health Monitoring}

Structural health monitoring, or SHM for short, is the process of establishing some knowledge of the current condition of a structure. The ultimate goal is to determine the existence, location, and degree of damage in a structure. A great deal of research in the past twenty years has been aimed at establishing effective methods in different settings. The efforts in the SHM research can be separated into two general groups, local SHM and global SHM. In order to convey an appreciation for the nature of the research in SHM, this section presents a brief description of a few of the techniques developed to detect the various forms of damage in both the global and local approaches. As the work presented in this paper is a type of global method, the issues relevant to related global methods are also discussed.

This survey is intended to provide a representative picture of some of the work being done in SHM. It is not meant to be a complete and in-depth exposition on all of the current research. For a more thorough treatment of the available SHM methods see Natke and Yao (1988), Agbabian and Masri (1988), IMAC (1997), and Masri (1997). 


\subsubsection{Local Structural Health Monitoring}

Local SHM techniques use direct examination of structural members to determine the condition of those members. Many methods fall into this class. Visual inspection is the most basic form of local SHM. This type of monitoring is capable of detecting only very obvious structural faults. Any small scale defects and problems in inaccessible structural members are difficult to detect. In order to assist with detecting small scale faults, non-destructive evaluation (NDE) techniques can be employed. Dye penetrant methods aim to enhance the visibility of surface cracks through the use of a coating on the surface of the structural member. Eddy current and magnetic flux approaches

look for cracks and delaminations (for composite materials) by monitoring impedance changes in a coil placed near the surface of the member. Radiography uses $\mathrm{X}$-rays or gamma-rays passed through the member to reconstruct an image of the member. The image can be studied for flaws. Ultrasonic techniques send high frequency sound into a member and measure the backscatter. The character of the backscatter will be affected by cracks, delaminations, and other defects. Numerous studies applying these methods to civil and aerospace applications have been conducted (CRC 1991; Burdekin 1993; Popovics and Rose 1994; Zalameda et al. 1994; Jenks et al. 1997). While these NDE methods provide a good picture of the member under consideration, there are a few limitations. First, the NDE techniques have the same problem as visual inspection in regard to inaccessible members. Also, applying these procedures to any more than a few structural members will be quite difficult and expensive. Finally, these approaches require an inspector to be present to conduct the investigation. This limits the frequency of inspection, which, as in the case of the Mianus River bridge, can prove to be fatal.

Another set of local SHM techniques, which do not require user interaction, have also been developed. Such methods address the problems of monitoring 
difficult-to-access parts of a structure and of increasing the rate of monitoring by placing sensors either directly on structural members or embedding them in the members and monitoring them remotely. A large contingent of these SHM approaches are based on using fiber optic sensors to sense strain or the presence of cracks in the members to which the sensors are attached. Some applications of fiber optic strain gauges to health monitoring can be found in Lyöri et al. (1994), Measures et al. (1994), Masri et al. (1994), Measures (1996), and deVries et al. (1996). Fan et al. (1995), Leung and Elvin (1996), and Chen et al. (1996) show methods for using an optic fiber attached to a structural member to directly determine the existence and location of cracks. For a more thorough presentation of the developments in these types of SHM techniques, Agbabian and Masri (1988), Claus (1991), Udd (1991), and Claus (1993) are good sources of information.

All of these local techniques can give an indication of the location and possible degree of damage in the area to which they are applied. By exhaustive application to an entire structure, these local methods could provide a very complete picture of the current damage state of the structure. For small regular structures such as pressure vessels and wing boxes, this might be a reasonable application of these local approaches. For structures beyond a certain size and complexity, examination of every part of the structure is not feasible. These methods are therefore best used to monitor specific parts of a structure.

\subsubsection{Global Structural Health Monitoring}

The global SHM approaches attempt to determine the overall damage state of a structure through measurement at only a few points in the structure. The benefit gained is that the condition of the whole structure can be monitored simultaneously. The drawback is that localizing and determining the degree 
of damage becomes more difficult. The key step in these methods is the use of some type of framework to relate changes in the measurements at particular points to changes in the overall structure. Different choices for the framework and the goals of the specific technique separate the various global methods.

\section{Direct Methods}

One approach involves using measurements from the structure by themselves to detect the existence of damage. In these methods, the following is a typical scenario. First, sets of structural data from likely damaged conditions are generated using different configurations of an analytical or experimental model. Pattern recognition techniques are then used to establish the existence and location of damage by comparing measured data to the pre-established patterns. The obvious limitation to these types of methods is that categorizing all possible damaged configurations is practically impossible.

Mazurek and DeWolf (1990) suggest using variations in the modeshapes and frequencies as indicators of structural deterioration. They note that in order to detect damage through variations in modal parameters, changes due to normal operation conditions must be taken into account. Also, if damage location is to be found, changes in modal parameters in different failure modes must be known. The study presents a series of laboratory tests aimed at determining these characteristics for a model bridge girder.

Hearn and Testa (1991) also present a method for determining damage by direct analysis of changes in frequencies. Their method uses an initial model of a structure to develop sets of ratios of changes in the frequencies for variations in different structural members. They propose using the set closest (in a least squares sense) to the observed ratio changes from measured data to indicate the existence and location of damage. Some experimental

tests of the suggested method using a welded steel frame and a wire rope are 
presented.

Use of neural networks has been also been considered. In these SHM applications, the neural network is trained to recognize patterns of behavior in the data which represent expected modes of structural degradation. Gerardi and Hickman (1991) present an application to aircraft structures which looks for rivet corrosion and ice accretion using the frequency response function as input. Mangal et al. (1996) apply a neural network to recognize stiffness loss in offshore jacket platforms through changes in the dynamic response. The network is trained with output from a finite-element model of the offshore platform. Ceravolo and Destefano (1995) use neural networks to determine damage in a truss model by examining variations in the modal frequencies. Strains are the input in a method developed for analysis of aircraft by Kudva et al. (1991). A finite element model under different damage configurations provides the training input.

\section{Structural Model-Based Inverse Methods}

The final type of SHM methods described in this section use variations in structural models identified from different measured data, rather than variations in the data itself, to determine the condition of the structure. The critical assumption is made that changes in the parameters of the structural model imply changes in the parts of the real structure associated with the parameters. These model-based SHM approaches rely on structural model updating (also known as system identification) methodologies to solve the inverse problem of determining the parameters of a model given some measured data. This is a fundamental difference from the other global methods, wherein any structural model is used only to suggest likely data under different dam-

aged states. A short discussion of model updating thus is necessary before describing the model-based SHM techniques. Model updating is a field unto 
itself with concerns and applications quite apart from SHM. Only the aspects relevant to the SHM problem are considered here.

Generally, model updating involves choosing the parameters of a model to minimize an objective function based on the error between the measured data and the corresponding quantities calculated from the model. Mottershead and Friswell (1993) present a very thorough survey on various model updating methods. All the model updating methods have three common aspects. First, there is noise in the measured data. In this work, "noise" will refer to variations of the measured modal data when there are no fundamental changes in the underlying structure. The effect of this noise is that model parameters identified from different data sets may be different, even if there is no change in the underlying structure. Another shared aspect of all the methods is that the models only approximate the real systems. No model, no matter how refined, is capable of completely duplicating the behavior of the structure it models in all loading conditions. Thus, model error is always present. Finally, even if there were no model error and no noise, the objective function used to compare the measured data and the corresponding quantities calculated from the model may be more sensitive to some model parameters and less sensitive to others. Small variations in a low sensitivity parameter might not significantly change the objective function. Thus, in some sense, that parameter is not well characterized by the model updating process.

The model error, noisy data, and low sensitivity to parameter variation combine to create a degree of uncertainty in the identified model parameters. If the degree of uncertainty is too large for a given application, then problems could arise in using the identified model parameters. As a simple illustration, suppose the effects of model error, noisy data and low sensitivity result in a degree of uncertainty such that the identified model parameter is very likely to have a value between 0.99 and 1.01 but is unlikely to have a value outside 
this range. If, in some application, knowing the value of the parameter to within 0.001 of the true value were critical, the uncertainty in this case would be a problem. On the other hand, in an application where the parameter only needs to be known to within 0.1 of the true value, the parameter uncertainty would not have a significant effect.

The uncertainty problem carries over into the model-based inverse SHM methods, although many methods do not explicitly address it. When the structure under consideration is well-characterized by the analytical model, many controlled measurements can be taken, and the measurements have very low noise levels, no significant uncertainty may be present, and ignoring it should not lead to any problems. However, in many cases, such as with civil structures, these assumptions do not apply. The analytical models rarely capture the full behavior of the structure. Further, the amount of measured information is limited. For instance, the modal parameters of only a few of the lower modes of a civil structure can generally be determined with confidence. Finally, when the measured modal data is available, it tends to show significant variation from one measurement to the next. Any SHM method applied in the case of civil structures should therefore account for substantial uncertainty in the identified model parameters.

On a very basic level, all of the model-based SHM techniques can be viewed as doing the same thing:

1. Construct a structural model whose parameters have nominal values selected by engineering judgment.

2. Measure data from the structure in an undamaged configuration and use a model updating procedure to get better estimates for the undamaged model parameters.

3. Measure data from the structure periodically and generate some metric 
for the variation with respect to the undamaged state.

4. Use the measure to detect changes in the structure.

The methods are differentiated by the structural model used, the type and amount of measured data necessary, what metric is used to detect damage, and whether or not the parameter uncertainty issue is considered. Restrictions on the amount of data will limit the number of parameters in the model that can be uniquely identified. Uncertainty in the identified model parameters will lead to difficulties in concluding whether changes in the model parameters are due to damage or an effect of noisy data, model error, and insensitivity. Thus, in order to properly address the SHM problem, a method should take into account the realistic situations of limited measurement information and parameter uncertainty.

Kim and Bartkowicz (1993), Kim et al. (1995), and Zimmerman et al. (1996) present an on-going effort using a combined modal expansion/model reduction approach for updating finite element models. Stiffness matrices are identified from different sets of modal data, and a direct comparison is made to determine the existence and location of damage. They have applied their method to theoretical models, an 8-bay experimental truss, and a $10^{-}$ bay experimental truss. Their results have been good, although they do not explicitly take the parameter uncertainty issue into account.

Another method which uses differences in identified stiffnesses is given in Topole and Stubbs (1995). Although these authors make the claim that their method is superior to others because it requires no initial modal data, they assume the initial mass and stiffness matrices are perfect matches to the structure being studied. Initial data of some type must be used to validate this supposition.

Farhat and Hemez (1993) and Hemez and Farhat (1995) present a method which updates the elastic modulus and cross-sectional area of truss members. 
Changes in these parameters between data sets are used to indicate damage. They introduce a criterion to select which parameters should be updated. This is a nice feature since detailed models can be maintained. Results are presented for an analytical analysis of a 10-bay truss. The performance of the method is good when a high density of sensors is used, but worsens as the number of sensors is reduced. They note that identified damage will often "spread" to neighboring elements which are not in reality damaged. The effects of noise in the measured data are not considered.

In Pandey and Biswas $(1994,1995)$ an interesting approach to detecting damage using updated flexibility matrices, rather than stiffness matrices, is proposed and experimentally tested. The authors use the fact that under certain restrictions, the flexibility matrix is better characterized than the stiffness matrix by the lower modes of vibration. They suggest comparing observed changes in the flexibility matrices identified from different data sets with characteristic patterns of change for specific damaged configurations derived using an initial finite-element model of a structure. Although the results appear promising, some of the limitations of the method weigh against them. First, the method requires that the modeshapes at a fairly large number of points be known. Also, the number of damage patterns which can be saved is limited. Finally, the authors make no mention of the effects of noise in the data on their results.

An attempt is made by Baruh and Ratan (1993) to include the effect of uncertainties due to model error and measurement noise. They use a residual vector based on the eigenequation error as an indicator of damage existence and location. They never actually update a model, which is a nice feature. The effects of model error and measurement noise on the residual vector are studied, although no systematic accounting for these effects is discussed.

Fares and Maloof (1997) present a method which is philosophically very 
similar to the one which will be presented in this study. The focus of their work is finding cracks in a plate using strain measurements, although the method they pose has more general applicability. They acknowledge that the measurements will be noisy and the model for the plate inexact. These factors are accounted for by framing the problem in a probabilistic manner. A procedure for establishing the plausible range of data for a given model is introduced. Measured data is compared with the plausible range. When the data fall outside of the plausible range a statistically significant number of times, an "anomaly" is detected. The method that is developed shows promise, and, as the authors themselves note, may benefit from treatment in a Bayesian framework.

Finally, a great deal of investigation in the SHM area has been done by $\mathrm{H}$. Natke and J. Yao (Natke and Yao 1988; Natke and Yao 1993; Yao and Natke 1994). Their collected works both present a general framework for SHM, and discuss the important issues associated with SHM such as uncertainty in identified parameters. Their papers and books provide excellent guidelines for the type of work that is necessary in order to address the problems of SHM.

\subsubsection{Local and Global Method Working Together}

Local and global SHM should be considered as complementary, rather than competing approaches. For example, embedded sensors could be used to monitor key areas of a structure and provide data to a global SHM algorithm. The results of the global analysis could then be used to provide guidance in looking for damage in parts of the structure not instrumented with the embedded sensors. A local NDE method would be applied at the locations indicated by the global method. The use of both types of SHM together helps to partially overcome the limits encountered when each are applied alone. The potential advantages of joint application need to be considered in research in both areas 
of SHM.

\subsection{What Does This Work Present?}

This work presents a methodology for SHM which falls into the class of global structure model-based inverse methods. The SHM method described builds on and incorporates theoretical development in SHM and system identification by J. L. Beck and his current and former students (Beck and Katafygiotis 1992; Beck 1991; Beck 1996; Papadimitriou et al. 1995). Efforts by M. B. Levine and her colleagues (Levine-West and Milman 1994; Levine-West et al. 1996) also provided some inspiration in certain aspects of this work.

The goals of the method presented in this study are defined in the problem statement in Table 1.1. These goals are accomplished by formulating the solution in a Bayesian probabilistic framework. The Bayesian setting facilitates accounting for uncertainties due to noise ${ }^{1}$ in the measured modal data and modeling error. Also, engineering judgment concerning a number of issues can be incorporated in a mathematically formal fashion. Finally, in the Bayesian approach, addition of new modal data is treated in a systematic fashion.

One important aspect of the SHM procedure in this work is that measured modal data is used to perform health monitoring. This enables the method to function, if necessary, using only data available through small-amplitude ambient vibration monitoring of structures. Ambient vibration monitoring measures the motion of a structure due to constantly occurring events such as wind loading, traffic loading and micro-tremors. Methods have been developed to extract modal parameters from this ambient time domain data (Beck et al. 1994; Beck et al. 1995; Rubin 1980; James et al. 1992). The ambient

\footnotetext{
${ }^{1}$ What is meant by "noise" in the modal data is explained further in Section 2.4.1.
} 
Given

- A structure

- A linear dynamic model of the structure

- Sets of measured, noisy, incomplete modal data from the structure in a known undamaged condition

- Sets of measured, noisy, incomplete modal measurements taken at a periodic interval from the structure in an unknown condition

Establish: - Measures which reflect the probability of the existence, location, and degree of damage while accounting for the model parameter uncertainty which arises due to modeling error and noise in the measured modal data.

- A way to incorporate new data as it is measured so that an automated monitoring system can be developed.

Table 1.1 Problem statement for the SHM method developed in this study. 
vibration approach to SHM has several advantages over methods which utilize forced vibration response to determine modal parameters. First, for the low amplitude excitations typically experienced during ambient motion, most structural systems are well characterized with linear models, which are easier to treat than nonlinear ones. Also, the danger of further damaging the structure while testing it is reduced. Finally, continuous ambient vibration tests can be performed at very low cost. For a permanently instrumented structure, this will facilitate the implementation of the SHM method as an automatic monitoring system.

Two key assumptions go into this SHM method. These are

1. Damage in the structure will affect the measured data to a sufficient extent to be able to characterize the damage.

2. Variations in a model of the structure identified using data from the structure in undamaged and damaged states can be taken to infer the existence, location and degree of changes in the structural components corresponding to the changed model components.

The first applies to any SHM method, and the second is specific to modelbased methods. If either of these statements is not true, then SHM cannot be performed with a model-based SHM method which uses measured data. While verifying these assumptions is an important part of validating the use of the SHM procedure, to do so properly requires experimental testing on full-scale structures. Some such testing has been done (Rubin 1980; Mazurek and DeWolf 1990; O'Leary et al. 1992), but to fully discuss these issues is beyond the scope of this work. Thus, the SHM method is developed under the assumption that these key statements are valid, but will require further work in applying it to actual structures for confirmation of the assumptions.

Chapter 2 presents the approach to SHM taken in this study. First a 
brief background on Bayesian probability is given in Section 2.2. Section 2.3 provides an outline of the necessary steps in formulating a Bayesian SHM scheme. The details of the method devised for this study are in Sections 2.4 and 2.5. A summary the Bayesian SHM technique is in Section 2.6.

The result of testing the capabilities and limitations of the SHM methodology are presented in Chapter 3. Chapter 3 has examples of application to computer-generated data from model buildings. A simple two degree of freedom shear structure is studied in Section 3.2 in order to explore some of the key issues of the SHM method in a fairly exhaustive manner. Section 3.3 contains an example of applying the SHM method to a ten degree of freedom shear structure. In this case, damage scenarios are considered, and the behavior of the SHM technique is analyzed.

Avenues of further investigation are discussed in Section 4.1. Limitations of the developed method are mentioned in Section 4.2. Finally, some closing remarks on the SHM method developed in this study are made in 4.3 . 


\section{Chapter 2}

\section{Theoretical Development}

\section{$2.1 \quad$ Introduction}

As established in the preceding section, structural health monitoring can be a useful tool in maintaining structural safety and performance. However, developing health monitoring methods is not a trivial task due to the associated difficulties such as model parameter uncertainty. Consider handling the problem in a simple deterministic framework. In such a setting, an objective function based on an error between the model and measured output is defined and the "best" model parameters are those which minimize this chosen function. Differences in the "best" model parameters found from different data sets are used to infer changes in the structure. Two problems arise in this scheme. First, there is no way to find how well the measured data defines the model parameters. The objective function could be very peaked, very flat, or anywhere in between around the "best" model parameters. Furthermore, the data itself may vary without any changes in the measure structure. These effects lead to differences in the "best" model parameters identified from different data sets which are not caused by changes in the structure. Thus, performing health monitoring would be difficult. A purely deterministic approach does not address the problems which arise due to parameter uncertainty. 
A Bayesian probabilistic framework provides a means by which to handle the issue of parameter uncertainty. It can reproduce the results of some of the deterministic methods, albeit with a slightly different interpretation. Further, it provides the machinery by which a measure of the probability of damage can be assessed. In what follows, a general outline of Bayesian structural health monitoring is described. Then, the particular method developed for this study is detailed. Once the setting is established, some of the implications and limitations will be analyzed in Chapter 3 using synthetic test data.

\subsection{Bayesian Probability}

The first step in developing the Bayesian structural health monitoring(SHM) method is introducing Bayesian probability. In a Bayesian setting, probability is not thought of as relative frequency of events over multiple trials. Rather, probability is viewed as the plausibility of one set of statements given another set of statements. The relative plausibilities of different statements with respect to a common statement can thus be determined. Two good references for the philosophy behind Bayesian probability are Jeffreys (1939) and Jaynes (1978). Box and Tiao (1992) present some of the mechanics associated with using the theory.

Mathematically, the foundation of Bayesian statistics is Bayes' theorem, which is expressed using conditional probabilities. In terms of conditional probabilities of statements $A, B$, and $C$, Bayes' theorem is

$$
P(A \mid B, C)=\frac{P(B \mid A, C) P(A \mid C)}{P(B \mid C)}
$$

where $P(X \mid Y)$ is the probability of statement $X$ conditional upon statement $Y$, and " $Y, Z$ " denotes the conjunction of two statements, $Y$ and $Z$. If $X$ and $Y$ are statements about the values of random variables, then Bayes' theorem 
can also be stated in terms of conditional probability density functions (PDFs), giving

$$
p(a \mid b, c)=\frac{p(b \mid a, c)}{p(b \mid c)} p(a \mid c)
$$

Here, $p(x \mid y)$ is the probability density function (PDF) for $x$ conditional upon $y$ where $p(x \mid y) \equiv \lim _{d x \rightarrow 0} \frac{P(x<X \leq x+d x \mid Y=y)}{d x}$. Thus, $a, b, c, x$, and $y$ are all numerical variables.

In words, Bayes' theorem states that the updated (a posteriori) PDF for $a$ conditional on $b$ and $c$ is equal to the PDF for $b$ conditional on $a$ and $c$ scaled by an initial (a priori) PDF for $a$ given $c$ and normalized by the probability (PDF) of $b$ given $c$. The updated PDF is used to make inferences about $a$. Any conclusions about $a$ are said to be conditional on $b$ and $c$.

From the total probability theorem,

$$
p(b \mid c)=\int p(b \mid a, c) p(a \mid c) d a=k^{-1}
$$

Thus, $p(b \mid c)$ is a constant with respect to $a$ which normalizes the integral of $p(a \mid b, c)$ over all possible $a$. Bayes' theorem can then be written as

$$
p(a \mid b, c)=k p(b \mid a, c) p(a \mid c)
$$

This form of Bayes' theorem will be used throughout the remainder of this work.

The PDF $p(b \mid a, c)$ is chosen to reflect the plausibility of $b$ given $a$ and $c$. The criteria used to select the function depend on the problem under consideration. The initial PDF, sometimes called the prior PDF, reflects the initial knowledge of the statement $a$ included in $c$. This function can be non-informative, in which case $c$ tells nothing about $a$ and is a constant. Strictly speaking, if the 
uncertain variable is modeled as unbounded such a function should only be flat in a finite region of interest and fall off away from the region so that the PDF will have a finite integral over an infinite domain. Such an initial PDF is termed locally non-informative. In the case that the initial PDF reflects that c provides more information about $a$, it is called informative. For example, if $a$ is the value of some parameter which must be between 0 and 2 , then a locally non-informative initial PDF would be zero outside of this range and flat within the range. A possible informative prior could arise from letting $b$ and $c$ include successive data sets and using the updated PDF for $a$ obtained using $c$ as the initial PDF when the data set $b$ is considered. In general, the initial PDF enables the Bayesian setting to account for the effects of past knowledge, based either on data or engineering judgment, in an explicit mathematical form.

\subsection{Outline of Bayesian SHM}

The Bayesian probabilistic framework is a tool very well suited to handling the SHM problem. As will be shown, a measure of the plausibility of structural damage is generated which can be used to make decisions concerning structural safety and inspection locations. Also, the Bayesian method developed in this work addresses the parameter uncertainty issue. Constructing a Bayesian probabilistic approach to solve the SHM problem requires several steps. After a brief general description of these steps in this section, the particular decisions

this study makes and the resulting SHM procedure will be discussed in detail in sections 2.4 and 2.5 .

The primary step comes in choosing a structure and determining what observable characteristics it possesses which can be used to determine damage. The observable quantities, referred to in this study as observable parameters, 
could consist of modal properties, displacement, velocity, and acceleration time histories, strains, or any set of observable structural values which would reflect structural damage. Due to uncertainties associated with measurement error, unknown loading, and related factors, the observable parameters are treated as uncertain variables. When the observable parameters are measured, the resulting values are referred to as measured data or simply data.

Once the structure and types of observable parameters are determined, a class of structural models is formulated. The model class is parameterized by the structural model parameters which reside in the structural model parameter space. The structural models are chosen so as to be capable of producing a set of quantities which is equivalent to, but not necessarily equal to, the observable parameters. Suppose there are no uncertainties associated with the structural model class or the observable parameters. Further, allow that the structural model parameters can be chosen so that the structural model provides an exact match to the measured data. Under these conditions, if structural model parameters found using different sets of data are different, the inference can be made that the structure has changed in some fashion. The location and degree of change might even be indicated if the structural model parameters correspond to structural quantities such as the elastic modulus or cross-sectional area of a specific beam. In reality, uncertainties in both the observable and structural model parameters are always present. Uncertainties in the structural model parameters arise due to model error, non-ideal materials, unknown boundary conditions, and other factors. Conclusions may still be drawn concerning structural changes based on changes in the structural model parameters. However, the uncertainties in the observable parameters and the structural model parameters must be incorporated.

The probabilistic aspect of the approach now comes into play. A class of probability models, $\mathcal{P}$, is selected. The probability models are parameterized by 
the probability model parameters which reside in the probability model parameter space. The probability models define a PDF for the observable parameters given a model from the structural model class. This PDF is conditional on the structural model, the structural model class, and the probability model class. In the Bayesian setting described in Section 2.2, this PDF is viewed as $p(b \mid a, c)$. Thus, in terms of the quantities used in Bayes' theorem defined in the preceding section, the structural and probability model parameters correspond to $a$ and the observable parameters correspond to $b$. The structural model class and the probability model class together make up $c$. The structural model parameters and probability model parameters taken together are referred to as model parameters. The model parameters reside in the union of the structural model parameter space and the probability model parameter space, called the model parameter space.

After measurements have been made on the structure, Bayes' theorem can be invoked to determine an updated PDF where model parameters are treated as uncertain and the observable parameters are replaced by the measured data. Not all of the structural and probability model parameters need to be treated as uncertain. For instance, the probability parameters may be assumed known while the structural model parameters are not. In this case, the updated PDF would be formulated for the structural model parameters alone. In order to use Bayes' theorem, the initial PDF on the uncertain model parameters is also selected.

The updated PDF provides a measure of the relative plausibility of the various values of the uncertain model parameters. Integrating the updated PDF over sets of parameter values in the model parameter space will give a measure of the plausibility of those sets of values given the measured data. The probabilities derived from different measured data sets can be used to establish measures for the plausibility of the existence, degree, and location 
of damage. Thus, the final step in developing the Bayesian Structural Health Monitoring method is choosing how the updated PDF will be used to measure the probability of the existence, location, and degree of damage in a structure given measured data from the structure. There will necessarily be subjective choices which require simulation and experimental testing to investigate the limitations.

This brief description of SHM in a Bayesian setting outlined the key steps in a very general manner. Sections 2.4 and 2.5 detail the approach taken in this study.

\subsection{Formulating the Updated PDF}

The development of the SHM method follows in a manner which parallels the general description given in section 2.3. This section describes the steps taken to formulate the updated PDF. Section 2.5 presents the SHM method which uses the updated PDF developed in this section.

\subsubsection{Measured Data}

Clearly, changes in the effective stiffness of structural members will effect the modal parameters. Thus, these may be used to detect damage. The observable parameters for this work therefore comprise the modal parameters of a structure for a limited number of modes identified from measurements taken at a restricted number of degrees of freedom (DOFs) of the structure. These limitations reflect most real-world situations in which there are relatively few sensors, noise in the measurement system, and limited dynamic range in the excitation of the structure which restrict the number of modes and the number of modeshape components which can be identified. The observable modal parameters are quantities that can be determined. The measured values of 
the observable modal parameters, or simply measured modal parameters, are the values of the observable modal parameters when they are determined.

In general, the observable modal parameters would be identified from measured time-domain data using any of the standard modal parameter identification methods such as ERA(Juang and Pappa 1985), polyreference(Vold and Rocklin 1982), or MODE-ID (Beck 1996). The modal parameters used in this study are found using MODE-ID. For a given time-domain data set, the output of MODE-ID is an optimal modal estimate for that data set. Throughout this work, the implied method for acquiring the modal parameters will be ambient vibration monitoring. The advantages of ambient vibration monitoring are mentioned in Section 1.3.

As mentioned in Section 1.2.2, any variations in the measured modal parameters which are not a result of changes in the underlying structure are called "noise". For the modal parameters, these variations are caused by a number of factors. The measured time-domain data can differ from the true motion because of electrical noise, limitations of the transducers, and the quantization of the signal. Also, the methods for extracting the modal parameters from the time-domain data may make assumptions which are not entirely valid. Such effects contribute to the observable modal parameters being uncertain.

The total number of different measured time-domain data sets is $N_{s}$. The $\mathrm{n}^{\text {th }}$ optimal modal estimate provides a modal model whose output best fits the $\mathrm{n}^{\text {th }}$ measured time-domain data in a least squares sense. The modal model can be viewed as the most probable model within a class of linear dynamic models based on a superposition of classical normal modes (Beck 1996). The modal estimate for the $\mathrm{n}^{\text {th }}$ set of time--domain data comprises $N_{m}$ measured frequencies of vibration, $\hat{\omega}_{r}(n)$, and associated measured incomplete modeshape vectors, $\hat{\psi}_{r}(n) \in \mathbb{R}^{N_{o}}$, where $r \in 1, \ldots, N_{m}$, and $N_{o}$ represents the number of observed degrees of freedom. Since the incomplete modeshapes 
can be scaled arbitrarily, they are given a unit Euclidean norm. This restricts them to lie on the boundary of the $N_{o}$ dimensional unit ball, $\partial B_{N_{o}}$. In reality, therefore, only $N_{m} N_{o}$ independent parameters are available with each set of measured modal parameters. For instance, when only one DOF is measured, the number of parameters equals the number of frequencies identified since the associated modeshapes are all single elements which are set to 1. For this study, a simplifying assumption has been made that the same number of modal parameters are identified from every time-domain data set. This is not, in general, a situation which will always be true.

All of the measured modal parameters for the $n^{\text {th }}$ time-domain data set are referred to by $\hat{\Upsilon}_{n}$. The collection of all such measured data sets is the data, $\mathcal{D}_{N_{s}}$ Thus,

$$
\mathcal{D}_{N_{s}}=\left\{\hat{\Upsilon}_{1}, \ldots, \hat{\Upsilon}_{N_{s}}\right\}
$$

This study will focus on the case where the modal data consists of some data from an initial or undamaged structure and other data from the same structure in a potentially damaged state. Suppose the first $N_{u d}$ sets are from the undamaged structure, and the remaining $N_{s}-N_{u d}$ are from the structure after it has been monitored and new data acquired. Even though the structure, not the data, is damaged, the terms undamaged data and potentially damaged data will be used to describe data from undamaged and potentially damaged structures, and will be denoted respectively by

$$
\mathcal{D}_{u d}=\left\{\hat{\Upsilon}_{1}, \ldots, \hat{\Upsilon}_{N_{u d}}\right\}
$$


and

$$
\mathcal{D}_{p d}=\left\{\hat{\Upsilon}_{N_{u d}+1}, \ldots, \hat{\Upsilon}_{N_{s}}\right\}
$$

If damage is positively detected, the measured data sets which come from the damaged structure will be differentiated from the other data sets by calling them $\mathcal{D}_{d}$.

Without the "a", the parameters will refer to the observable modal parameters or observable modes. The set of all the observable modal parameters is referred to as $\Upsilon$.

\subsubsection{Structural Model Class}

The structural model class in this study, $\mathcal{M}_{N_{d}}$, consists of $N_{d}$ degree of freedom (DOF) linear systems with a known mass matrix which have dynamic behavior characterized by the equation of motion:

$$
M \ddot{x}+C(\theta) \dot{x}+K(\theta) x=f(t)
$$

with $f, x \in \mathbb{R}^{N_{d}}$, and $M, C, K \in \mathbb{R}^{N_{d} \times N_{d}}$. Furthermore, the model class has "expanded" modeshapes, which are explained later in this section.

\section{Defining the Fundamental Model}

The motion of a particular physical structure can be modeled by an equation of motion

$$
M \ddot{x}+\bar{C} \dot{x}+\bar{K} x=f(t) .
$$

One way to derive this relationship is through the finite-element method to model the structure as a multi-degree of freedom linear system possessing 
classical normal modes of vibration. Each element in the finite-element model has a contribution to the mass, damping, and stiffness matrices. The equation could also be formulated by assuming a simple model which consists of using discrete masses, damping elements, and linear spring elements to model the structure. Like the elements in the finite element model, each of these elements contributes to the overall mass, damping and stiffness matrices. In each approach, the elements of the model correspond to structural members. The mass matrix, $M$, derived in this fashion is assumed to be well-known and unchanging over time. Thus, models with varying mass are not part of the model class. A further fact of importance is that the mass matrix will be symmetric and, for the applications considered in this work, positive definite. The damping is assumed to be of a form such that $C M^{-1} K=K M^{-1} C$, which insures that the model possesses classical normal modes (Caughey and O'Kelly 1965).

The contribution from a finite element or a discrete spring element to the overall stiffness matrix, $\bar{K}$, is $\bar{K}_{i} \in \mathbb{R}^{N_{d} \times N_{d}}$. Thus, the stiffness matrix is of the form

$$
\bar{K}=\sum_{i=1}^{N_{e}} \bar{K}_{i}
$$

where $N_{e}$ is the number of elements in the model. A grouping of elements is called a substructure. A substructure's stiffness contribution to the overall stiffness matrix is given by the sum of the elemental stiffness matrices for the elements which make up the substructure. The substructure stiffness contributions can be grouped further into those which are considered known and those which are uncertain. Thus, the stiffness matrix can be written as

$$
\widetilde{K}=K_{0}+\sum_{i=1}^{N_{\theta}} K_{i}
$$


where $K_{0}$ is the sum of substructure stiffness contributions from the known set, and the $K_{i}$ are from the uncertain set.

\section{Parameterizing the Model Class}

The structural model class, $\mathcal{M}_{N_{d}}$, is defined in part as a set of models parameterized by introducing the vector of non-dimensional structural stiffness parameters $\theta \in \Theta \subseteq \mathbb{R}^{N_{\theta}}$ and defining $K(\theta)$ in (2.8) as

$$
K(\theta)=K_{0}+\sum_{i=1}^{N_{\theta}} \theta_{i} K_{i}
$$

The damping matrix, $C(\theta)$, is of a form such that the system given by (2.8) possesses classical normal modes. Since the stiffness parameters must remain positive, $\Theta$, the structural parameter space, consists of only positive values. Furthermore, the material properties and structural geometry could limit the range of values for the $\theta_{i}$. These model dependent properties would further constrain the parameter space. The model which provides the $K_{i}$ is called the fundamental model. This is essentially the model of the structure formed using analytical techniques alone. When $\theta=[1, \ldots, 1]^{T}$, the fundamental model is selected from $\mathcal{M}_{N_{d^{*}}}$ This fact will be used later in the justification for choosing an initial PDF for $\theta$.

The chosen parameterization for the model class preserves the structural connectivity of the fundamental model since an element of the fundamental stiffness matrix $\bar{K}$ which is zero remains zero in $K(\theta)$. This is desirable in structural health monitoring since changes in $\theta$ identified from successive data sets can then be related to changes in the real structure. However, unless a structural model parameter scales an individual elemental stiffness matrix, a change in $\theta$ reflecting variation only in that element is not part of $\mathcal{M}_{N_{d}}$. In fact, a change in the value of $\theta_{i}$ could be viewed as an equal change in all of 
the stiffnesses of the elements constituting the $\mathrm{i}^{\text {th }}$ substructure. In practice, this is seen to produce cases where the correct model parameters change to indicate the gross location of damage, but the magnitude of change is less than if individual elements were scaled.

This problem may be addressed with adaptive substructuring, wherein substructures suspected of having changed are re-substructured, and those whose values have not changed are lumped into the known set of substructures. A formal procedure to automatically perform the adaptive substructuring is beyond the scope of this work. This is a possible topic for future study in the Bayesian setting. Some techniques for adaptive substructuring for use in model updating have been developed for deterministic settings (Hjelmstad and Shin 1997).

Another advantage of substructuring is that the number of necessary model parameters is reduced. This is beneficial in two ways. First, it decreases the computation required when performing calculations to evaluate the parameters. Also, if the amount of measured data is limited, the number of parameters which can be found without incurring excessive non-uniqueness problems is limited.

\section{The Model Modal Parameters}

As is well known, for classical normal modes, the frequencies, $\omega_{s}(\theta)$, and mode shapes, $x_{s}(\theta)$, for the model are given, respectively, by the eigenvalues and the eigenvectors of

$$
\left[K(\theta)-\omega_{s}^{2}(\theta) M\right] x_{s}(\theta)=0
$$


The modeshapes are orthogonal with respect to $M$. Thus,

$$
x_{s}(\theta)^{T} M x_{t}(\theta)=\delta_{s t} \quad s, t \in 1, \ldots, N_{d}
$$

where $\delta_{s t}$ is the Kronecker delta function. Since the scaling on the modeshapes is arbitrary, they are taken to be mass normalized. With this scaling,

$$
\omega_{s}^{2}(\theta)=x_{s}(\theta)^{T} K(\theta) x_{s}(\theta)
$$

The matrix of all the model modeshapes is a full rank matrix which will be called $X(\theta)$. Using the results just described,

$$
X^{T}(\theta) M X(\theta)=I
$$

and

$$
X^{T}(\theta) K X(\theta)=\Omega^{2}(\theta)
$$

where $\Omega^{2}(\theta)$ is a diagonal matrix with the squares of the model frequencies on the diagonal. These relationships will be used extensively in the development of the updated PDF for the model parameters. Unless needed for clarity, the explicit dependence of these quantities on $\theta$ will be dropped.

For any given set of measured modal data, $\hat{\Upsilon}_{n}$, the number of identified modes, $N_{m}$, is much less than $N_{d}$, the number of degrees of freedom of the structural model. Also, the number of modeshape components which are identified for each mode, $N_{o}$, is typically much less than $N_{d}$ because a limited number of sensors is employed. The $N_{o}$ DOFs of the model corresponding to the observable modeshape parameter components are referred to as observed degrees of freedom. The observed degrees of freedom are selected from the full set of model degrees of freedom by the observation matrix, $\Gamma \in \mathbb{R}^{N_{o} \times N_{d}}$. 
Each row of the observation matrix possesses a single " 1 " in the appropriate location. The remaining components in the row are set to 0 . There is one such row in $\Gamma$ for each observed DOF.

\section{The Expansion Parameters}

As previously noted, the full set of modeshapes for a model in $\mathcal{M}_{N_{d}}$ spans the space $\mathbb{R}^{N_{d}}$. However, because of the mathematical structure built into the model, the model modeshapes cannot be arbitrarily chosen. It might therefore be possible that no element of $\mathcal{M}_{N_{d}}$ can produce modeshapes which match the measured modeshapes. In order to address this problem, the structural model class is "expanded" by taking linear combinations of the model modeshapes,

$$
\phi_{r}\left(\theta, \alpha_{r}\right)=X(\theta) \alpha_{r}, \quad r \in 1, \ldots, N_{m},
$$

for each observable mode. The $\phi_{r} \in \mathbb{R}^{N_{o}}$ are called expanded modeshapes. The vector of scalings on the model modeshapes, $\alpha_{r} \in \mathbb{R}^{N_{d}}$, are expansion vectors. All the expansion vectors taken together as a vector are called the expansion parameters and given the symbol $\alpha$. The expansion parameters $\alpha$ together with the structural parameters $\theta$ fully parameterize the model class, $\mathcal{M}_{N_{d^{*}}}$

For each observable mode, there is a corresponding set of expansion parameters. Therefore, the expanded model class has the potential for matching any set of classical normal modes.

As with the model modeshapes, the expansion vectors are known only to within a scaling factor. Thus, they are given a unit Euclidean norm. This restricts $\alpha_{r}$ to lie on the boundary of the $N_{d}$ dimensional unit ball, $\partial B_{N_{d}}$. This choice for scaling means that

$$
\phi_{r}^{T} M \phi_{r}=1
$$




\section{A Simple Model Class}

As an example of a model class, take $\mathcal{M}_{2}$ to be "The set of two DOF shear structure models with known mass." A picture of typical model is shown in Figure 2.1. The lumped masses are $m_{1}$ and $m_{2}$. The elemental stiffness

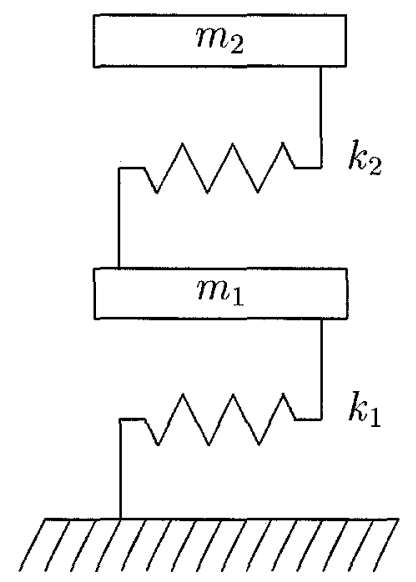

Figure 2.1 This is a representation of a two DOF shear structure model. The spring elements represent resistance to lateral motion between the "floors". The masses are assumed lumped at the "floors".

matrices are

$$
\bar{K}_{1}=\left[\begin{array}{cc}
k_{1} & 0 \\
0 & 0
\end{array}\right] \quad \text { and } \quad \bar{K}_{2}=\left[\begin{array}{cc}
k_{2} & -k_{2} \\
-k_{2} & k_{2}
\end{array}\right]
$$

Two possibilities exist for substructuring: one or two substructures. In the first case, $K_{1}=\bar{K}_{1}+\bar{K}_{2}$ and

$$
K=\theta_{1} K_{1}
$$


In the second case, $K_{1}=\bar{K}_{1}, K_{2}=\bar{K}_{2}$, and

$$
K=\theta_{1} K_{1}+\theta_{2} K_{2}
$$

Thus, there are either one or two structural parameters depending on the substructuring chosen. Prior knowledge of the properties of the members in the structure being modeled could put limits on the achievable stiffnesses, thus defining $\Theta$. The number of expansion parameters would depend on the number of modes measured. If only one mode were measured, $\alpha_{1} \in \mathbb{R}^{2}$ would be the only expansion vector. If two modes were measured, an additional expansion vector, $\alpha_{2} \in \mathbb{R}^{2}$, would be added. This model class is used extensively during the simulated data testing in Chapter 3.

\subsubsection{Probability Model Class}

Recall, that the observable modal parameters are unknown quantities. The deterministic models in $\mathcal{M}_{N_{d}}$ are selected to be capable of producing a set of model modal parameters which are equivalent to, but not necessarily equal to, the observable modal parameters. If there were no modeling error and no expected noise in the observable parameters when measured, then the observable modal parameter could be considered equal to the model modal parameters. However, model error is present in any real situation since no model is capable of fully characterizing the behavior of a structure. Also, noise in the measured modal parameters will always be present to some degree for reasons discussed in Section 2.4.1. Thus, the observable modal parameters can only be characterized in a probabilistic sense.

The Probability Model Class, $\mathcal{P}$, establishes a probability distribution for the observable parameters given the structural, expansion and probability parameters. The number of probability parameters depends on the form of the 
probability models. As mentioned in Section 2.3, the combined set of structural, expansion, and probability parameters is referred to as the model parameters. The probability distribution is chosen to reflect the potential modeling error and the uncertainty in the observable parameters when they are measured. These are subjectively chosen to model a state of incomplete knowledge, although there are some guidelines such as the Principle of Maximum Entropy (Jaynes 1978) that suggest possible good choices.

Before defining the PDF for the observable parameters, a few assumptions are made. First, the observable parameters are assumed to be independently distributed from mode to mode and from frequency to modeshape. Also, the components of a given modeshape are not independently distributed from each other. These assumptions are equivalent to stating that knowing the value of one observable frequency or modeshape does not relate any information about the value of another observable frequency or modeshape.

With these assumptions, the PDF for the modeshape and frequency of a single observable mode can be formulated separately. The complete PDF will be a product of the individual frequency and modeshape PDFs over all the observable modes. The modeshape PDF is considered first. Then the frequency PDF is developed.

Unless noted, all of the PDFs defined will have a conditional dependence on the model class, $\mathcal{M}_{N_{d}}$, and the probability model class, $\mathcal{P}$. This conditional dependence will be explicitly stated during the first definition of a PDF, but dropped thereafter in order to shorten the notation. Also, all norms are Euclidean norms unless noted. 


\section{Modeshape PDF}

Choose as the PDF for $\psi_{r}$ given $\theta, \alpha_{r}, \epsilon_{\psi_{r}}, \mathcal{M}_{N_{d^{\prime}}}$ and $\mathcal{P}$

$p\left(\psi_{r} \mid \theta, \alpha_{r}, \epsilon_{\psi_{r}}, \mathcal{M}_{N_{d}} \mathcal{P}\right) \equiv k_{r} \exp \left[-\frac{1}{2} \frac{1}{\epsilon_{\psi_{r}}^{2}}\left\|\frac{\psi_{r}}{\left\|\psi_{r}\right\|}-\frac{\left\langle\psi_{r}, \Gamma X \alpha_{r}\right\rangle}{\left\|\psi_{r}\right\|\left\|\Gamma X \alpha_{r}\right\|} \frac{\Gamma X \alpha_{r}}{\left\|\Gamma X \alpha_{r}\right\|}\right\|^{2}\right]$.

Recall from Section 2.4.1 that $\psi_{r}$ is the ${ }^{\text {th }}$ observable modeshape. The $\mathrm{PDF}^{1}$ can also be expressed in terms of $\theta$ and $\phi_{r}$ as

$$
p\left(\psi_{r} \mid \theta, \alpha_{r}, \epsilon_{\psi_{r}}, \mathcal{M}_{N_{d}} \mathcal{P}\right) \equiv k_{r} \exp \left[-\frac{1}{2} \frac{1}{\epsilon_{\psi_{r}}^{2}}\left\|\frac{\psi_{r}}{\left\|\psi_{r}\right\|}-\frac{\left\langle\psi_{r}, \Gamma \phi_{r}\right\rangle}{\left\|\psi_{r}\right\|\left\|\Gamma \phi_{r}\right\|} \frac{\Gamma \phi_{r}}{\left\|\Gamma \phi_{r}\right\|}\right\|^{2}\right]
$$

In this PDF, the error between the normalized observed modeshape and a scaled version of the corresponding normalized observed expanded modeshape, $\Gamma \phi_{r}$, has been modeled as a Gaussian distribution with zero mean and a diagonal covariance matrix with $\epsilon_{\psi_{r}}^{2}$ for all of the diagonal elements. The Principle of Maximum Entropy (Jaynes 1978) can be used as a justification for this choice of a PDF. The analysis which follows shows that the probability parameter $\epsilon_{\psi_{r}}$ can also be interpreted as a measure of the degree of uncertainty in the angle between $\psi_{r}$ and $\Gamma \phi_{r}$. This work will not treat $\epsilon_{\psi_{r}}$ as an uncertain parameter. A criterion for its selection is developed in Section 2.4.4. Determining the behavior for unknown $\epsilon_{\psi_{r}}$ will be briefly mentioned later in this section. The scaling on the $\Gamma \phi_{r}$ can be shown to be the optimal scaling on the vector in the sense that it minimizes the norm of the error with respect to the scaling. A proof of this statement is presented in appendix A.

\footnotetext{
${ }^{1}$ Strictly speaking, this second form of the PDF is a different function, $\tilde{p}\left(\psi_{r} \mid \tilde{\theta}, \phi_{r}, \epsilon_{\psi_{r}}\right)$, where setting $\tilde{\theta}=\theta$ and $\phi_{r}\left(\theta, \alpha_{r}\right)=X \alpha_{r}$ gives $p=\tilde{p}$. Such subtleties will not be treated in this study. For the remainder of this work, the implicit dependence of functions on the expansion parameters when they are written in terms of the expanded modeshapes is assumed.
} 
By manipulating the algebra in equation 2.24 and using the fact that $\psi_{r}$ has a unit norm, the conditional PDF for the observable modeshapes can be expressed in two different forms.

$$
\begin{aligned}
& p\left(\psi_{r} \mid \theta, \alpha_{r}, \epsilon_{\psi_{r}}\right)=k_{r} \exp \left[-\frac{1}{2} \frac{1}{\epsilon_{\psi_{r}}^{2}} \sin ^{2} \beta_{r}\right] \\
& p\left(\psi_{r} \mid \theta, \alpha_{r}, \epsilon_{\psi_{r}}\right)=k_{r} \exp \left[-\frac{1}{2} \frac{1}{\epsilon_{\psi_{r}}^{2}} \frac{\phi_{r}^{T} \Gamma^{T}\left(I-\psi_{r} \psi_{r}^{T}\right) \Gamma \phi_{r}}{\left\|\Gamma \phi_{r}\right\|^{2}}\right] .
\end{aligned}
$$

Here, $\beta_{r}$ is the angle between $\psi_{r}$ and $\Gamma \phi_{r}$ given by

$$
\cos \beta_{r}=\frac{\left\langle\psi_{r}, \Gamma \phi_{r}\right\rangle}{\left\|\psi_{r}\right\|\left\|\Gamma \phi_{r}\right\|}
$$

The chosen PDF for the observable modeshape thus depends only on $\beta_{r}$. This fact can be used to show that the constant $k_{r}$ does not depend on $\theta$ or $\alpha_{r}$.

Since $k_{r}$ is the normalizing constant for the PDF,

$$
k_{r}^{-1}=\int_{\partial B_{N_{o}}} \exp \left[-\frac{1}{2} \frac{1}{\epsilon_{\psi_{r}}^{2}} \sin ^{2} \beta_{r}\right] d A
$$

where $\partial B_{N_{o}}$ is the surface of the $N_{o}$-dimensional unit sphere and $d A$ is an area element on this surface. Recall that $\psi_{r}$ has been set to have a unit norm. On $\partial B_{N_{o}}, \psi_{r}$ can be expressed in terms of $N_{o}-1$ angles. For $N_{o}=1$ this means that there is no PDF for the measured modeshapes. This is consistent with the fact that with one DOF measured, there is no available modeshape information. Let one of the angles be $\beta_{r}$, defined previously as the angle between $\psi_{r}$ and $\Gamma \phi_{r}$. This step is allowed since $\Gamma \phi_{r}$ can be viewed as one of the coordinate axes. The integrand does not depend on the $N_{o}-2$ other angles so they may be integrated out. The integral over $\beta_{r}$ will be from $-\pi$ to $\pi$. It has a value dependent on $\epsilon_{\psi_{r}}$, but not $\theta$ or $\alpha_{r}$. Thus, $k_{r}$ does not depend on $\theta$ or $\alpha_{r}$. Actually, calculating the value of $k_{r}$ is not necessary since it will 
be canceled when the updated PDF for $\theta$ and $\alpha$ is formed.

The dependence of $k_{r}$ on $\epsilon_{\psi_{r}}$ is complicated in general. However, for values of $\epsilon_{\psi_{r}}<0.2$, the dependence of $k_{r}$ on $\epsilon_{\psi_{r}}$ closely follows the function $2 \sqrt{2 \pi} \epsilon_{\psi_{r}}$. This result follows from the fact that the integrand in (2.27) is well approximated by a Gaussian density function in $\beta_{r}$ for such values of $\epsilon_{\psi_{r}}$. This fact may be helpful in the case where the $\epsilon_{\psi_{r}}$ are treated as uncertain parameters. This treatment is left for future work. For the remainder of this work, the explicit conditional dependence will be dropped in the notation and left as implicit in order to simplify the notation.

The expression for the observable modeshape PDF given by (2.25b) will be useful for computational purposes. Derivations involving this form for the PDF will lead to expressions which simplify the computations for the SHM method.

\section{Frequency PDF}

Choosing the PDF for the observable frequencies is more problematic. Like the model modeshape, the model frequencies may not precisely correspond to observable frequencies. Unlike the observable modeshapes case, taking a linear combination of the model frequencies as a mean frequency and assuming a probability distribution using the resulting frequency error between the observable and mean frequencies does not appear to be an obvious step. However, there should be some reflection of the weighting represented by $\alpha_{r}$ in the frequency part of the PDF. In order to accomplish this, and to reflect uncertainty in the relationship between model frequencies and observable frequencies, the PDF for the $\mathrm{r}^{\text {th }}$ measured frequency is defined as a product of $N_{d}$ different Gaussian distributions on $\omega_{r}^{2}$ with means given by the $N_{d}$ model eigenvalues, standard deviations $\sigma_{\omega_{r}^{2}}$, and weighting by the square of the corresponding component from $\alpha_{r}$. The $\sigma_{\omega_{r}^{2}}$, like the $\epsilon_{\psi_{r}}$, will be found in a deterministic 
fashion. The choices of these values is discussed in Section 2.4.4.

$$
\begin{gathered}
p\left(\omega_{r}^{2} \mid \theta, \alpha_{r}, \sigma_{\omega_{r}^{2}}, \mathcal{M}_{N_{d}}, \mathcal{P}\right) \equiv \frac{1}{f_{r}\left(\theta, \alpha_{r}, \sigma_{\omega_{r}^{2}}\right)} \prod_{s=1}^{N_{d}} \exp \left[-\frac{1}{2} \frac{1}{\sigma_{\omega_{r}^{2}}^{2}} \frac{\alpha_{r s}^{2}\left(\omega_{r}^{2}-\omega_{s}^{2}(\theta)\right)^{2}}{\alpha_{r}^{T} \alpha_{r}}\right] \\
=\frac{1}{f_{r}\left(\theta, \alpha_{r}, \sigma_{\omega_{r}^{2}}\right)} \exp \left[-\frac{1}{2} \frac{1}{\sigma_{\omega_{r}^{2}}^{2}} \frac{1}{\alpha_{r}^{T} \alpha_{r}} \sum_{s=1}^{N_{d}} \alpha_{r s}^{2}\left(\omega_{r}^{2}-\omega_{s}^{2}(\theta)\right)^{2}\right]
\end{gathered}
$$

The function $f_{r}\left(\theta, \alpha_{r}, \sigma_{\omega_{r}^{2}}\right)$ is a normalizing factor so that

$$
\int_{0}^{\infty} p\left(\omega_{r}^{2} \mid \theta, \alpha_{r}, \sigma_{\omega_{r}^{2}}, \mathcal{M}_{N_{d}} \mathcal{P}\right) d\left(\omega_{r}^{2}\right)=1
$$

This choice for the PDF of $\omega_{r}^{2}$ reflects the initial uncertainty concerning which model frequencies correspond to which observable frequencies.

Using the terminology associated with $\mathcal{M}_{N_{d}}$ defined in section $2.4 .2^{2}$, the product of exponentials can be transformed into two more convenient and informative forms. The details of the algebra involved are in appendix A.

$$
\begin{array}{r}
p\left(\omega_{r}^{2} \mid \theta, \alpha_{r}, \sigma_{\omega_{r}^{2}}\right)=\frac{1}{f_{r}\left(\theta, \alpha_{r}, \sigma_{\omega_{r}^{2}}\right)} \exp \left[-\frac{1}{2} \frac{1}{\sigma_{\omega_{r}^{2}}^{2}}\left(\left(\omega_{r}^{2}-\frac{\alpha_{r}^{T} \Omega^{2} \alpha_{r}}{\alpha_{r}^{T} \alpha_{r}}\right)^{2}+\right.\right. \\
\left.\left.\frac{\left(\alpha_{r}^{T} \Omega^{4} \alpha_{r}\right)}{\alpha_{r}^{T} \alpha_{r}}-\frac{\left(\alpha_{r}^{T} \Omega^{2} \alpha_{r}\right)^{2}}{\left(\alpha_{r}^{T} \alpha_{r}\right)^{2}}\right)\right]
\end{array}
$$

$$
p\left(\omega_{r}^{2} \mid \theta, \alpha_{r}, \sigma_{\omega_{r}^{2}}\right)=\frac{1}{f_{r}\left(\theta, \alpha_{r}, \sigma_{\omega_{r}^{2}}\right)} \exp \left[-\frac{1}{2} \frac{1}{\sigma_{\omega_{r}^{2}}^{2}} \frac{1}{\left\|\phi_{r}\right\|_{M}^{2}}\left\|\left(K-\omega_{r}^{2} M\right) \phi_{r}\right\|_{M^{-1}}^{2}\right]
$$

\footnotetext{
${ }^{2}$ The matrix $\Omega(\theta)$ is a diagonal matrix of natural frequencies of the structural model given by $\theta$. The matrix $\Omega$ is related to the stiffness matrix by

$$
\Omega^{2}(\theta)=X^{T}(\theta) K(\theta) X(\theta)
$$
}

where $X(\theta)$ is the mass normalized $\left(X^{T} M X=I\right)$ matrix of model modeshapes. 
Equations, (2.29) and (2.31) imply that

$$
f_{r}\left(\theta, \alpha_{r}, \sigma_{\omega_{r}^{2}}\right)=\frac{1}{\sqrt{2 \pi} \sigma_{\omega_{r}^{2}}} \exp \left[-\frac{1}{2} \frac{1}{\sigma_{\omega_{r}^{2}}^{2}}\left(\frac{\left(\alpha_{r}^{T} \Omega^{4} \alpha_{r}\right)}{\alpha_{r}^{T} \alpha_{r}}-\frac{\left(\alpha_{r}^{T} \Omega^{2} \alpha_{r}\right)^{2}}{\left(\alpha_{r}^{T} \alpha_{r}\right)^{2}}\right)\right]
$$

and the PDF for $\omega_{r}^{2}$ becomes

$$
p\left(\omega_{r}^{2} \mid \theta, \alpha_{r}, \sigma_{\omega_{r}^{2}}\right)=\frac{1}{\sqrt{2 \pi} \sigma_{\omega_{r}^{2}}} \exp \left[-\frac{1}{2} \frac{1}{\sigma_{\omega_{r}^{2}}^{2}}\left(\omega_{r}^{2}-\tilde{\omega}_{r}^{2}\right)^{2}\right]
$$

Here,

$$
\begin{aligned}
\tilde{\omega}_{r}^{2}\left(\theta, \alpha_{r}, \sigma_{\omega_{r}^{2}}\right) & =\frac{\phi_{r}^{T} K \phi_{r}}{\phi_{r}^{T} M \phi_{r}} \\
& =\sum_{s=1}^{N_{d}} \alpha_{r s}^{2} \omega_{s}^{2}(\theta)
\end{aligned}
$$

Technically, (2.34) gives a non-zero probability that $\omega_{r}^{2}<0$, which cannot be true. Generally, however, $\sigma_{\omega_{r}^{2}}$ is sufficiently small that for all practical purposes, the probability volume for $\omega_{r}^{2}<0$ is negligible. The term in equation (2.35a) is the Rayleigh quotient for the $K-M$ system using the expanded modeshapes as the test vector. This is also seen to be a weighted sum of the model eigenvalues. The PDF for the frequencies is therefore a Gaussian distribution with a mean given by a weighted sum of the model squared-frequencies and a variance given by $\sigma_{\omega_{r}^{2}}^{2}$.

The other form of the PDF, (2.32), possesses a term in the exponential which is the eigenvalue equation error with a modeshape given by $\phi_{r}$ and frequency given by the observable frequency. The scaling function can also be written in terms of $K$ and $M$ (details in appendix $\mathrm{A})$ :

$$
f_{r}\left(\theta, \alpha_{r}, \sigma_{\omega_{r}^{2}}\right)=\frac{1}{\sqrt{2 \pi} \sigma_{\omega_{r}^{2}}} \exp \left[-\frac{1}{2} \frac{1}{\sigma_{\omega_{r}^{2}}^{2}} \frac{1}{\left\|\phi_{r}\right\|_{M}^{2}}\left\|\left(K-\tilde{\omega}_{r}^{2} M\right) \phi_{r}\right\|_{M^{-1}}^{2}\right]
$$


The term in the exponential in equation (2.36) is also an eigenvalue equation error. This second equation error is of the same form as the first, but uses the Rayleigh quotient in place of the observable frequency. This term is negative semidefinite, achieving zero if and only if $\phi_{r}$ is an eigenvector for the structural model.

Since the probability parameters are considered known, the conditional dependence on them will only be explicitly expressed in the notation when necessary for clarification. Otherwise, the dependence will be left implicit.

\section{The Complete PDF for the Observable Parameters}

The complete PDF for the observable parameters conditional upon the model parameters, structural model class and probability model class is the product all the individual observable modeshape and frequency PDFs given by (2.24) or $(2.25 \mathrm{~b})$ and (2.32) or (2.34) respectively. The result upon combining terms is

$$
\begin{aligned}
p\left(\Upsilon \mid \theta, \alpha, \epsilon, \sigma, \mathcal{M}_{N_{d}} \mathcal{P}\right) & =k(\epsilon, \sigma) \exp \left[-\frac{1}{2} Q(\theta, \alpha)\right] \\
& =\frac{k(\epsilon, \sigma)}{f(\theta, \alpha, \sigma)} \exp \left[-\frac{1}{2} R(\theta, \alpha)\right]
\end{aligned}
$$

where

$$
Q(\theta, \alpha)=\sum_{r=1}^{N_{m}}\left(\frac{1}{\sigma_{\omega_{r}^{2}}^{2}}\left(\omega_{r}^{2}-\tilde{\omega}_{r}^{2}\right)^{2}+\frac{1}{\epsilon_{\psi_{r}}^{2}}\left\|\frac{\psi_{r}}{\left\|\psi_{r}\right\|}-\frac{\left\langle\psi_{r}, \Gamma \phi_{r}\right\rangle}{\left\|\psi_{r}\right\|\left\|\Gamma \phi_{r}\right\|} \frac{\Gamma \phi_{r}}{\left\|\Gamma \phi_{r}\right\|}\right\|^{2}\right)
$$

$$
R(\theta, \alpha)=\sum_{r=1}^{N_{m}}\left(\frac{1}{\sigma_{\omega_{r}^{2}}^{2}} \frac{\left\|\left(K-\omega_{r}^{2} M\right) \phi_{r}\right\|_{M^{-1}}^{2}}{\left\|\phi_{r}\right\|_{M}^{2}}+\frac{1}{\epsilon_{\psi_{r}}^{2}} \frac{\phi_{r}^{T} \Gamma^{T}\left(I-\psi_{r} \psi_{r}^{T}\right) \Gamma \phi_{r}}{\left\|\Gamma \phi_{r}\right\|^{2}}\right)
$$


In (2.37), the parameters $\epsilon$ and $\sigma$ refer to sets of the $\epsilon_{\psi_{r}}$ and $\sigma_{\omega_{r}^{2}}$, respectively,for all of the $N_{m}$ modes.

\subsubsection{Updated PDF for the Model Parameters}

Let the structure be monitored over time and $N_{s}$ measurements made of the observable parameters. Modal data, $\mathcal{D}_{N_{s}}$, is then available. The updated PDF based on all of the data is

$$
p\left(\theta, \alpha \mid \mathcal{D}_{N_{s}}, \mathcal{T}_{N_{s}}\right)=p\left(\theta, \alpha \mid \hat{\Upsilon}_{1}, \ldots, \hat{\Upsilon}_{N_{s}}, \mathcal{T}_{N_{s}}\right)
$$

Applying Bayes' Theorem (2.4) gives

$$
p\left(\theta, \alpha \mid \mathcal{D}_{N_{s}}, \mathcal{T}_{N_{s}}\right)=\tilde{k} p\left(\theta, \alpha \mid \mathcal{T}_{N_{s}}\right) p\left(\mathcal{D}_{N_{s}} \mid \theta, \alpha, \mathcal{T}_{N_{s}}\right)
$$

where $\tilde{k}$ is a normalizing constant and $p\left(\theta, \alpha \mid \mathcal{T}_{N_{s}}\right)$ is the conditional initial distribution on the model parameters. Using the axiom of probability that $p(a, b \mid c)=p(a \mid b, c) p(b \mid c), p\left(\mathcal{D}_{N_{s}} \mid \theta, \alpha, \mathcal{T}_{N_{s}}\right)$ can be written as

$$
\begin{aligned}
p\left(\mathcal{D}_{N_{s}} \mid \theta, \alpha, \mathcal{T}_{N_{s}}\right)= & p\left(\hat{\Upsilon}_{N_{s}} \mid \mathcal{D}_{N_{s}-1}, \theta, \alpha, \mathcal{T}_{N_{s}}\right) p\left(\hat{\Upsilon}_{N_{s}-1} \mid \mathcal{D}_{N_{s}-2}, \theta, \alpha, \mathcal{T}_{N_{s}}\right) \cdots \\
& p\left(\hat{\Upsilon}_{1} \mid \theta, \alpha, \mathcal{T}_{N_{s}}\right) \\
= & \prod_{n=1}^{N_{s}} p\left(\hat{\Upsilon}_{n} \mid \mathcal{D}_{n-1}, \theta, \alpha, \mathcal{T}_{N_{s}}\right)
\end{aligned}
$$

The updated PDF for the model parameters can then be expressed as

$$
p\left(\theta, \alpha \mid \mathcal{D}_{N_{s}}, \mathcal{T}_{N_{s}}\right)=\tilde{k} p\left(\theta, \alpha \mid \mathcal{T}_{N_{s}}\right) \prod_{n=1}^{N_{s}} p\left(\hat{\Upsilon}(n) \mid \mathcal{D}_{n-1}, \theta, \alpha, \mathcal{T}_{N_{s}}\right)
$$

The term $\mathcal{T}_{N_{s}}$ represents information that the data were derived from the 
structure at different times and knowledge as to the state of the structure with respect to the different measured data sets $\hat{\Upsilon}_{n}$ in $\mathcal{D}_{N_{s}}$. For instance, the information may represent that the first $N_{u d}$ measured data sets came from a structure without damage, but the condition of the structure for the remaining $N_{s}-N_{u d}$ sets is unknown. Using $\mathcal{T}_{N_{s}}$, two subsets of $\mathcal{D}_{N_{s}}$ could be formed, one containing only the undamaged data, $\mathcal{D}_{u d}$, and the other the potentially damaged data, $\mathcal{D}_{p d}$. This situation was also described in Section 2.4.1 and will be used in the development of the SHM method in Section 2.5.

More generally, this concept of forming subsets of the total set of data can be used to separate the data from different operational conditions. For example, a bridge might exhibit varying behavior with changes in traffic patterns, temperatures, and wind loading. The measured data taken from a single condition would be grouped together with other measured data taken in the same condition. This grouping of data could then be used to facilitate tracking of normal variations in the structure due to changing environmental influences. The general construction suggested here gives a greater degree of flexibility than is necessary for the application described in this study. However, it may be used in future work to account for the problems associated with monitoring time-varying structures mentioned in Section 4.1. While the explicit conditional dependence on $\mathcal{T}_{N_{s}}$ in expressions is maintained in this section, it will be dropped in future sections in order to simplify the notation.

As a notational aid, the selection function, $s(n), n \in 1, \ldots, N_{s}$ is introduced. Each subset of $\mathcal{D}_{N_{s}}$ has a selection function. The selection function is 1 for each $\hat{\Upsilon}(n)$ in $\mathcal{D}_{N_{s}}$ which is also in the subset, and 0 otherwise. Essentially, the selection function is used in the development which follows to write expressions with respect to $\mathcal{D}_{N_{s}}$ and $s(n)$ rather than writing different ones for each subset of $\mathcal{D}_{N_{s}}$. As an example, for the subsets $\mathcal{D}_{u d}$ and $\mathcal{D}_{p d}, s_{u d}(n)$ and 
$s_{p d}(n)$ would be defined where

$$
s_{u d}(n)=\left\{\begin{array}{cc}
1 & \text { if } n \leq N_{u d} \\
0 & \text { otherwise }
\end{array}\right.
$$

and

$$
s_{p d}(n)= \begin{cases}1 & \text { if } n>N_{u d} \\ 0 & \text { otherwise }\end{cases}
$$

The application of the selection function will be made clearer once it is used in the following section.

Finding $p\left(\hat{\Upsilon}_{n} \mid \mathcal{D}_{n-1}, \theta, \alpha, \mathcal{T}_{N_{s}}\right)$

The PDF $p\left(\hat{\Upsilon}_{n} \mid \mathcal{D}_{n-1}, \theta, \alpha, \mathcal{T}_{N_{s}}\right)$ is assumed to be independent of $\mathcal{D}_{n-1}$ and have the same form as the PDF given in (2.37). The only difference between $p\left(\hat{\Upsilon}_{n} \mid \mathcal{D}_{n-1}, \theta, \alpha, \mathcal{T}_{N_{s}}\right)$ and the PDF given in (2.37) is that the former PDF will have an additional conditional dependence on $\mathcal{T}_{N_{s}}$. Thus,

$$
p\left(\hat{\Upsilon}_{n} \mid \mathcal{D}_{n-1}, \theta, \alpha, \mathcal{T}_{N_{s}}\right)=p\left(\hat{\Upsilon}_{n} \mid \theta, \alpha, \mathcal{T}_{N_{s}}\right)
$$

where $p\left(\hat{\Upsilon}_{n} \mid \theta, \alpha, \mathcal{T}_{N_{s}}\right)$ is given by (2.37). Combining (2.46) and (2.43), the PDF on all of the data can be expressed as

$$
p\left(\theta, \alpha \mid \mathcal{D}_{N_{s}}, \mathcal{T}_{N_{s}}\right)=\tilde{k} p\left(\theta, \alpha \mid \mathcal{T}_{N_{s}}\right) \prod_{n=1}^{N_{s}} p\left(\hat{\Upsilon}_{n} \mid \theta, \alpha, \mathcal{T}_{N_{s}}\right)
$$

The updated PDF on all of the data is thus simply product of the updated PDFs for each of the measured sets taken individually. Letting $R_{n}(\theta, \alpha)$ be the function given in (2.39) corresponding to the $\mathrm{n}^{\text {th }}$ measured data set, $(2.47)$ 
becomes

$$
\begin{aligned}
p\left(\theta, \alpha \mid \mathcal{D}_{N_{s}}, \mathcal{T}_{N_{s}}\right) & =\hat{k} p\left(\theta, \alpha \mid \mathcal{T}_{N_{s}}\right) \prod_{n=1}^{N_{s}} \frac{1}{f(\theta, \alpha, \sigma)} \exp \left[-\frac{1}{2} R_{n}(\theta, \alpha)\right] \\
& =\hat{k} p\left(\theta, \alpha \mid \mathcal{T}_{N_{s}}\right) \frac{1}{f(\theta, \alpha, \sigma)^{N_{s}}} \exp \left[-\frac{1}{2} \sum_{n=1}^{N_{s}} R_{n}(\theta, \alpha)\right] .
\end{aligned}
$$

Consider the situation when the PDF based on the data in a subset of $\mathcal{D}_{N_{s}}$ is needed. Let the selection function for the subset be $s(n)$. The PDF on the data in the subset is then given by

$$
p\left(\theta, \alpha \mid \mathcal{D}_{N_{s}}, \mathcal{T}_{N_{s}}\right)=\hat{k} p\left(\theta, \alpha \mid \mathcal{T}_{N_{s}}\right) \frac{1}{f(\theta, \alpha, \sigma)^{\sum_{n=1}^{N_{s}} s(n)}} \exp \left[-\frac{1}{2} \sum_{n=1}^{N_{s}} s(n) R_{n}(\theta, \alpha)\right] .
$$

Clearly, (2.48) is a special case of (2.49). As mentioned when the selection function was defined, it is a convenience to aid in simplifying the notation. The relation in (2.49) could also have been written as a product in which the undesired PDFs where merely dropped.

\section{Calculating the Probability Parameters}

The information $\mathcal{T}_{N_{s}}$ is used to determine $\sigma$ and $\epsilon$ for a given subset of $\mathcal{D}_{N_{s}}$. Let the selection function for the subset be $s(n)$. The values of $\sigma_{\omega_{r}^{2}}$ and $\epsilon_{\psi_{r}}$ for this subset are then

$$
\sigma_{\omega_{r}^{2}}=\epsilon_{\omega_{r}^{2}} \bar{\omega}_{r}^{2}
$$

and

$$
\epsilon_{\psi_{r}}^{2}=\frac{1}{\sum_{n=1}^{N_{s}} s(n)} \sum_{n=1}^{N_{s}} s(n) \frac{\left\|\hat{\psi}_{r}(n)-\bar{\psi}_{r}\right\|^{2}}{\left\|\hat{\psi}_{r}(n)\right\|^{2}},
$$


where $\bar{\omega}_{r}^{2}$ is the mean of $\hat{\omega}_{r}^{2}(n)$,

$$
\bar{\omega}_{r}^{2}=\frac{1}{\sum_{n=1}^{N_{s}} s(n)} \sum_{n=1}^{N_{s}} s(n) \hat{\omega}_{r}^{2}(n)
$$

$\epsilon_{\omega_{r}^{2}}$ is the standard deviation of $\hat{\omega}_{r}^{2}(n)$,

$$
\epsilon_{\omega_{r}^{2}}=\frac{1}{\bar{\omega}_{r}^{2}} \sqrt{\frac{1}{\left(\sum_{n=1}^{N_{s}} s(n)\right)-1} \sum_{n=1}^{N_{s}} s(n)\left(\omega_{r}^{2}(n)-\bar{\omega}_{r}^{2}\right)^{2}}
$$

and $\bar{\psi}_{r}$ is the mean value of $\hat{\psi}_{r}(n)$,

$$
\bar{\psi}_{r}=\frac{1}{\sum_{n=1}^{N_{s}} s(n)} \sum_{n=1}^{N_{s}} s(n) \frac{\hat{\psi}_{r}(n)}{\left\|\hat{\psi}_{r}(n)\right\|}
$$

all taken over the measured data sets specified by $s(n)$.

For subsets which contain only one measured data set, the $\epsilon_{\omega_{r}^{2}}$ and $\sigma_{\omega_{r}^{2}}$ cannot be calculated as above. In this case, the coefficients of variation for the single selected data set are taken to be the same as those used for another subset. The mean frequencies are simply the values of the new measured frequencies. If no data are yet measured, no objective statistics are available for selecting the probability parameters. In this case, engineering experience and intuition are used to select them. Such choices are not very critical in the SHM problem, since the assumption has been made that many measurements will be taken on the structure in a "healthy" state before the SHM method is fully implemented. 


\section{Choosing an Initial PDF For $\alpha$}

The initial PDF can be factored so that

$$
p\left(\theta, \alpha \mid \mathcal{T}_{N_{s}}\right)=p\left(\alpha \mid \theta, \mathcal{T}_{N_{s}}\right) p\left(\theta \mid \mathcal{T}_{N_{s}}\right)
$$

where $p\left(\alpha \mid \theta, \mathcal{T}_{N_{s}}\right)$ is the conditional initial PDF for the expansion parameters and $p\left(\theta \mid \mathcal{T}_{N_{s}}\right)$ is the initial PDF for the model parameters. When no measured modeshape data is available, there is no information to indicate how much different the model modeshapes are from the observable modeshapes. On these grounds, the assumption is made that in the absence of measured modeshape data, the most likely $\alpha_{r}$ should be those which give expanded modeshapes equal to the model modeshapes (i.e. $\alpha_{r}=e_{r}$ where $e_{r} \in \mathbb{R}^{N_{d}}$ is a vector with the $r^{\text {th }}$ element equal to one, and all other equal to zero). Without an initial distribution on $\alpha_{r}$, this assumption may not be satisfied by the updated PDF.

In order to demonstrate this violation, consider modeling a simple physical structure using the two-DOF model class case described at the end of Section 2.4.2. Let the observable parameters consist of only one frequency, $\omega_{1}$, measured at a single time. In this case, $\alpha=\left[a_{1} a_{2}\right]^{T}$, where $a_{1}, a_{2} \in \mathbb{R}$. As a result of the norm constraint on the expansion parameters, $a_{1}=\cos (\beta)$ and $a_{2}=\sin (\beta)$ with $-\pi<\beta \leq \pi$ completely describes the set of possible values for $\alpha$. Let the prior on $\alpha$ be non-informative. The updated PDF as a function of $\alpha$ will then simply be a scaled version of the modal PDF which, for a fixed choice of structural parameters, is only a function of $\beta$. For this simple case, taking $\theta$ fixed, the updated PDF can be written explicitly as

$$
\begin{aligned}
p(\alpha \mid \theta) & =\tilde{k}(\theta) \exp \left[-\frac{1}{2} \frac{1}{\sigma_{\omega_{1}^{2}}^{2}}\left(\hat{\omega}_{1}^{2}-a_{1}^{2} \omega_{1}^{2}(\theta)-a_{2}^{2} \omega_{2}^{2}(\theta)\right)^{2}\right] \\
& =\tilde{k}(\theta) \exp \left[-\frac{1}{2} \frac{\hat{\omega}_{1}^{4}}{\sigma_{\omega_{1}^{2}}^{2}}\left(1-\frac{\omega_{1}^{2}(\theta)}{\hat{\omega}_{1}^{2}} \cos ^{2} \beta-\frac{\omega_{2}^{2}(\theta)}{\hat{\omega}_{1}^{2}} \sin ^{2} \beta\right)^{2}\right] .
\end{aligned}
$$


For a representative choice of $\theta$ and $\sigma_{\omega_{1}^{2}}$ the PDF in (2.56) is plotted as a function of $\beta$ in Figure 2.2. This plot displays four sharp peaks. Two of the

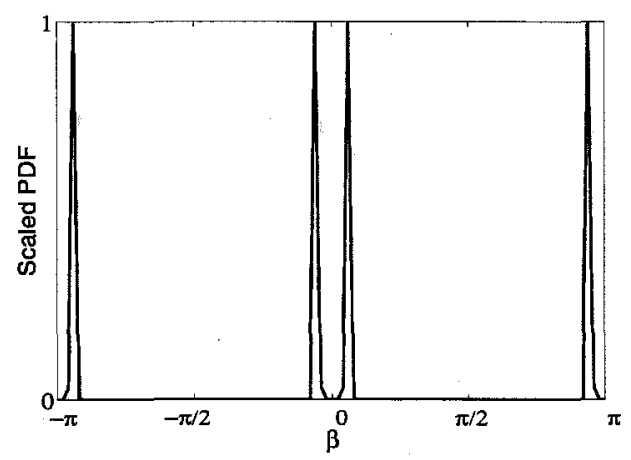

Figure 2.2 This is a plot of a scaled updated PDF versus $\beta$ for the a two-DOF model class, with one frequency measured and a non-informative prior for $\alpha$.

peaks correspond to $\beta$ 's which are $\pi$ variations from the other corresponding $\beta$ 's. Thus, only two different values for $\alpha$ are really represented. Given the extremely peaked nature of the plot, the conclusion could be drawn that the $a_{1}(\beta)$ and $a_{2}(\beta)$ which correspond to the peaks are the most likely values of the expansion parameters. Thus, in the absence of any measured modeshape data, not only do the solutions violate the assumption made concerning the values of the expansion parameters when no measured modeshape information is available, multiple solutions for the expansion parameters exist. By looking at (2.37a), these effects can clearly be seen to arise due to the freedom of the weighted mean term, $\tilde{\omega}_{r}$. As the example just given shows, in certain cases, $\alpha_{r} \neq e_{r}$ can be chosen so that $\tilde{\omega}_{r}=\hat{\omega}_{r}$.

In order to correct this situation, assume the $\alpha_{r}$ to be independently distributed from each other, and take as the priors on each of the $\alpha_{r}$

$$
p\left(\alpha_{r} \mid \theta, \mathcal{T}_{N_{s}}\right)=c_{r}(\theta) f_{r}\left(\theta, \alpha_{r}, \sigma_{\omega_{r}^{2}}\right)^{\sum_{n=1}^{N_{s}} s(n)}
$$


These initial distributions will remove the function $f()$ from (2.49). Since the term in the exponential in $f_{r}$ (see (2.33)) is negative definite, achieving zero if and only if the expanded modeshapes correspond to structural model modeshapes, the PDF on $\alpha_{r}$ can be seen as reflecting a prior assumption that the expanded modeshapes should be the model modeshapes. The function $c_{r}(\theta)$ is chosen so that the resulting updated PDF is normalized. When this PDF is used in the previously discussed two-DOF case, and $\theta$ is again considered fixed, the form of the updated PDF on $\alpha$ expressed in (2.58) is derived.

$$
\begin{aligned}
p(\alpha \mid \theta) & =k(\theta) \exp \left[-\frac{1}{2} \frac{1}{\sigma_{\omega_{1}^{2}}^{2}}\left(\left(\hat{\omega}_{1}^{2}-\omega_{1}^{2}(\theta)\right)^{2} a_{1}^{2}+\left(\hat{\omega}_{1}^{2}-\omega_{2}^{2}(\theta)\right)^{2} a_{2}^{2}\right)\right] \\
& =k(\theta) \exp \left[-\frac{1}{2} \frac{1}{\sigma_{\omega_{1}^{2}}^{2}}\left(\left(\hat{\omega}_{1}^{2}-\omega_{1}^{2}(\theta)\right)^{2} \cos ^{2} \beta+\left(\hat{\omega}_{1}^{2}-\omega_{2}^{2}(\theta)\right)^{2} \sin ^{2} \beta\right)\right]
\end{aligned}
$$

Taking for $\theta$ and $\sigma_{\omega_{1}^{2}}$ the same choices as in the previous case, the PDF in (2.58) is plotted as a function of $\beta$ in Figure 2.3. The plot shows that most of the

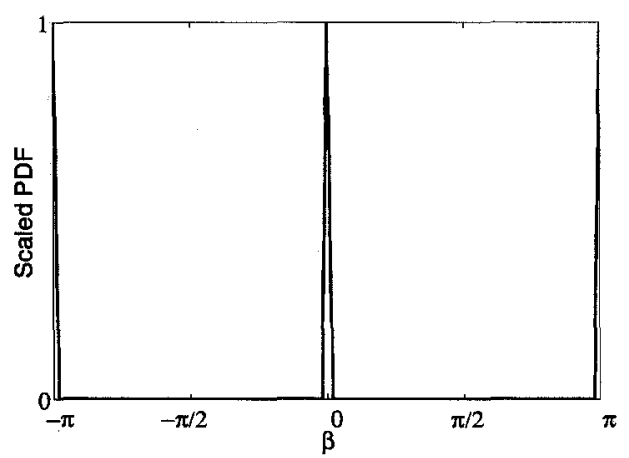

Figure 2.3 This is a plot of a scaled updated PDF versus $\beta$ for the a two-DOF model class, with one frequency measured and the informative prior for $\alpha$ given in (2.57).

probability volume is located near $\beta=0$ and $\beta=\pi$. These correspond to 
$\alpha_{r}=e_{1}$ and $\alpha_{r}=-e_{1}$ respectively. This can be used to infer that the structural model modeshape associated with the first mode is the most probable expanded modeshape.

In general, the following claim can be made concerning the updated PDF with the initial PDF on $\alpha$ described in (2.57).

Claim 2.1 Given the initial PDF on the expansion parameters chosen in (2.57), and the fact that no measured modeshape information is available, each expanded modeshape which maximizes the updated PDF will correspond to either a single structural model modeshape, or an arbitrary linear combination of a fixed set of structural model modeshapes.

Proof: When no modeshape information is available, the second term in the observed modal parameter PDF (2.39) is zero. If (2.49) and (2.57) are combined while holding $\theta$ fixed, the resulting updated PDF on $\alpha$ is

$$
p(\alpha \mid \theta)=k(\theta) \sum_{n=1}^{N_{s}} \frac{s(n)}{\left\|\phi_{r}\right\|_{M}^{2}}\left\|\left(K-\hat{\omega}_{r}^{2}(n) M\right) \phi_{r}\right\|_{M^{-1}}^{2}
$$

Based on the second form of the frequency PDF in (2.28) and the form given in $(2.32)$,

$$
\sum_{n=1}^{N_{s}} \frac{s(n)}{\left\|\phi_{r}\right\|_{M}^{2}}\left\|\left(K-\hat{\omega}_{r}^{2}(n) M\right) \phi_{r}\right\|_{M^{-1}}^{2}=\frac{\alpha_{r}^{T}\left(\sum_{n=1}^{N_{s}} s(n)\left(\hat{\omega}_{r}^{2}(n) I-\Omega^{2}\right)^{2}\right) \alpha_{r}}{\alpha_{r}^{T} \alpha_{r}}
$$

The right-hand side of (2.60) is a Rayleigh quotient. Since all of the matrices involved are diagonal, the conclusion can immediately be drawn that the minimizing expansion vectors correspond to $e_{s}$, where $s$ are location of the minimum diagonal values. If the diagonal elements are distinct, then only one such minimum exists. The minimizing $\alpha_{r}$ is then $e_{s}$, which makes $\phi_{r}$ the $\mathrm{s}^{\text {th }}$ 
structural model modeshape.

If the diagonal elements are not distinct, and the minimum element is repeated, then $\alpha_{r}$ is an arbitrary linear combination of the appropriate $e_{s}$. Thus, $\phi_{r}$ is any linear combination of the corresponding structural model modeshape vectors.

The figures in this section display a characteristic behavior of the updated PDF with respect to the expansion parameters. The sharp peaks of the updated PDF as a function of the expansion parameters occur over a wide range of structural model parameters. This is one justification for the approximation used later in this section to calculate the integral over the expansion parameters.

\section{Choosing an Initial PDF For $\theta$}

Before the experimental parameters are observed, any knowledge of the structural parameters is reflected in the initial conditional PDF $p\left(\theta \mid \mathcal{T}_{N_{s}}\right)$. This choice could reflect how confident the analyst is in the finite element modeling procedure. A high degree of confidence would lead to an initial PDF that is peaked with respect to some region in the structural parameter space. Low confidence would be indicated by a non-peaked, or perhaps even a locally flat, initial distribution.

In this study the choice of a prior is made based on an additional criterion. The normalization functions $c_{r}(\theta)$ introduced by the initial PDFs for the expansion parameters present problems because they are difficult to determine and lead to a form for the updated PDF that is not desirable. Therefore, the initial PDF for the structural parameters is selected to be $\pi\left(\theta \mid \mathcal{T}_{N_{s}}\right) / \prod_{r=1}^{N_{m}} c_{r}(\theta)$. This PDF cancels the $c_{r}(\theta)$ terms introduced by the selection of the initial distribution for $\alpha$ given in (2.57), and leaves the possibility for further selection of a prior on $\theta$ through $\pi\left(\theta \mid \mathcal{T}_{N_{s}}\right)$. 
In this work,

$$
\pi\left(\theta \mid \mathcal{T}_{N_{s}}\right)=g(\theta) \mathcal{N}\left(\theta_{F}, S\right)
$$

where

$$
\mathcal{N}\left(\theta_{F}, S\right) \sim \exp \left[-\frac{1}{2}\left(\theta-\theta_{F}\right)^{T} S^{-1}\left(\theta-\theta_{F}\right)\right]
$$

is a joint Gaussian distribution with mean $\theta_{F} \in \mathbb{R}^{N_{\theta}}$ and a covariance matrix $S \in \mathbb{R}^{N_{\theta} \times N_{\theta}}$. The choice for $\theta_{F}$ will generally be $[1, \ldots, 1]^{T}$ which reflects the belief that the fundamental model is the most probable model in the absence of any data. The individual parameters will be assumed to be independent, making $S$ a diagonal matrix of variances:

$$
S=\left[\begin{array}{lll}
\sigma_{1}^{2} & & \\
& \ddots & \\
& & \sigma_{N_{\theta}}^{2}
\end{array}\right] .
$$

The choices for the $\sigma_{i}$ reflect the initial level of uncertainty in the fundamental model. Technically, (2.62) gives a non-zero probability that $\theta_{i}<0$, which cannot be true. To address this problem, for damage detection purposes Beck and Katafygiotis (1992) suggest a two-sided initial distribution with a lognormal distribution for $0<\theta_{i} \leq 1$ and a Gaussian distribution for $\theta>1$. The purely Gaussian initial distribution will be shown to have a more convenient mathematical form than the two-sided distribution, so it is the initial distribution chosen. The non-zero problem is addressed by selecting the initial variances so that the probability volume for $\theta_{i}<0$ is negligible. The need for $g(\theta)$ is explained when the marginal distribution on $\theta$ is formed. 


\section{Final Form of the Updated PDF}

The final form of the updated conditional PDF for $\theta$ and $\alpha$ for the subset of $\mathcal{D}_{N_{s}}$ defined by the selection function $s(n)$ is given by combining $(2.37 \mathrm{~b})$, (2.39), (2.43), (2.55), (2.57), (2.61), and (2.62) to get

$$
\begin{aligned}
& p\left(\theta, \alpha \mid \mathcal{D}_{N_{s}}, \mathcal{T}_{N_{s}}\right)=\tilde{k} \exp {\left[-\frac{1}{2} \sum_{r=1}^{N_{m}} \sum_{n=1}^{N_{s}}\left(\frac{s(n)}{\sigma_{\omega_{r}^{2}}^{2}} \frac{\left\|\left(K-\hat{\omega}_{r}^{2}(n) M\right) \phi_{r}\right\|_{M-1}^{2}}{\left\|\phi_{r}\right\|_{M}^{2}}+\right.\right.} \\
&\left.\left.\frac{s(n)}{\epsilon_{\psi_{r}}^{2}} \frac{\phi_{r}^{T} \Gamma^{T}\left(I-\psi_{r}(n) \psi_{r}^{T}(n)\right) \Gamma \phi_{r}}{\left\|\Gamma \phi_{r}\right\|^{2}}\right)\right] \mathcal{N}\left(\theta_{F}, S\right) g(\theta) .
\end{aligned}
$$

Let the modal measure of fit (MMOF), $J_{r}\left(\theta, \alpha_{r}\right)$, be defined by

$$
J_{r}\left(\theta, \alpha_{r}\right)=\sum_{n=1}^{N_{s}} s(n)\left[\frac{\left\|\left(K-\hat{\omega}_{r}^{2}(n) M\right) \phi_{r}\right\|_{M^{-1}}^{2}}{\sigma_{\omega_{r}^{2}}^{2}\left\|\phi_{r}\right\|_{M}^{2}}+\frac{\phi_{r}^{T} \Gamma^{T}\left(I-\psi_{r}(n) \psi_{r}^{T}(n)\right) \Gamma \phi_{r}}{\epsilon_{\psi_{r}}^{2}\left\|\Gamma \phi_{r}\right\|^{2}}\right]
$$

This is called the modal measure of fit since it represents a measure of the error between measured and structural model modal parameters for a given measured mode. Based on the theoretical development thus far, the first term in the summation in equation (2.65) can be viewed as a frequency error and the second term as a modeshape component error.

The overall measure of fit (MOF), $J(\theta, \alpha)$, will be

$$
J(\theta, \alpha)=\left(\theta-\theta_{F}\right)^{T} S^{-1}\left(\theta-\theta_{F}\right)+\sum_{n=1}^{N_{m}} J_{r}\left(\theta, \alpha_{r}\right)
$$

Using this, the updated PDF can be written

$$
p\left(\theta, \alpha \mid \mathcal{D}_{N_{s}}, \mathcal{T}_{N_{s}}\right)=\tilde{k}(\epsilon, \sigma) \exp \left[-\frac{1}{2} J(\theta, \alpha)\right] g(\theta) .
$$


In the absence of the term $g(\theta)$, the maxima of the updated PDF are the minima of the MOF.

\section{Determining the Marginal Distribution on $\theta$}

Since the $\alpha$ are not used in the SHM method which will be developed, only the marginal distribution on the $\theta$ is needed. The marginal distribution on $\theta$ is given by integrating (2.64) over all possible $\alpha$. Performing this integration analytically is not possible. Therefore, an asymptotic approximation to the integral is made.

The integration procedure, described in Papadimitriou et al. (1995), takes two steps. First, the PDF is approximated by a Gaussian distribution about the maximum. Then, Laplace's method of asymptotic expansion is used to obtain

$$
p\left(\theta \mid \mathcal{D}_{N_{s}}, \mathcal{T}_{N_{s}}\right) \approx(2 \pi)^{\frac{N}{2}} \tilde{k} \exp \left[-\frac{1}{2} J(\theta, \hat{\alpha}(\theta))\right] \frac{g(\theta)}{\sqrt{\operatorname{det}\left[L_{\alpha}(\theta)\right]}}
$$

where $L_{\alpha}(\theta)$ is the Hessian matrix of second derivatives of $J(\theta, \alpha)$ with respect to $\alpha$ evaluated at $\hat{\alpha}(\theta)$. When the eigenvalues of the Hessian matrix are large, as they tend to be for the expansion parameters, this approximation is very good. The quantity $\hat{\alpha}(\theta)$ is the $\alpha$ that minimizes $J(\theta, \alpha)$ for fixed $\theta$. This is found by solving

$$
\frac{\partial J(\theta, \alpha)}{\partial \alpha_{r}}=0
$$

for $r \in 1, \ldots, N_{m}$. The optimal expanded modeshapes, $\hat{\phi}_{r}, r \in 1, \ldots, N_{m}$, are defined, where

$$
\hat{\phi}_{r}=X \hat{\alpha}_{r}
$$


Since the mapping $X$ will always have full rank,

$$
\frac{\partial J(\theta, \alpha)}{\partial \alpha_{r}}=0 \Leftrightarrow \frac{\partial J(\theta, \alpha)}{\partial \phi_{r}}=0
$$

The function of $\theta$ introduced in this process, $L_{\alpha}(\theta)$, is removed from the marginal distribution by selecting $g(\theta)$ to be $\sqrt{\operatorname{det}\left[L_{\alpha}(\theta)\right]}$. This is equivalent to choosing part of the initial PDF on $\theta$ so that the integrand in the PDF approximation is a normal Gaussian distribution with respect to $\alpha$.

This entire approximation process could be looked at in a different fashion which would lead to the same result, but in a less mathematically oriented fashion. Recall that the expansion parameters are incorporated in the model class so that any set of classical normal modes can be achieved by elements of the model class. Thus, for a given $\theta$ and set of measured data, the $\alpha$ can be viewed as providing the proper linear combination of model modeshapes so that some measure of the difference between the modal parameters for the model and the measured ones is minimized. If the MOF is used as the measure, then the same results follow as for the probabilistic framework.

Using either approach, the marginal updated PDF on $\theta$ becomes

$$
p\left(\theta \mid \mathcal{D}_{N_{s}}, \mathcal{T}_{N_{s}}\right)=\tilde{k} \exp \left[-\frac{1}{2} \tilde{J}(\theta)\right]
$$

where

$$
\tilde{J}(\theta)=J(\theta, \hat{\alpha}(\theta))
$$

This function, $\tilde{J}(\theta)$ is also called the measure of fit. For the remainder of this chapter, only the marginal distribution will be used. Thus, "updated PDF" will be used to mean "marginal updated PDF." Some results relating the derivatives of $\tilde{J}(\theta)$ to those of $J(\theta, \hat{\alpha}(\theta))$ can be found in Appendix B. 
The structural model parameters which give the maximum of (2.72) are

referred to by $\hat{\theta}$, and can be viewed as the most probable models given the data. Necessarily, $\hat{\theta}$ also minimize $\tilde{J}(\theta)$. In a strictly deterministic setting, the analysis would end at calculating $\hat{\theta}$ for a given $\tilde{J}(\theta)$. Changes in $\hat{\theta}$ between some reference undamaged state and the current state would be used to determine the health of the structure. The additional information on the uncertainty in the identified most probable models contained in (2.72) would be ignored. The SHM procedure presented in the following section uses this information to account for the uncertainties due to noise and modeling error.

\subsection{Bayesian SHM Framework}

This work proposes a measure derived from the marginal distributions on the individual structural model parameters using updated PDFs conditional on undamaged, $\mathcal{D}_{u d}$, and potentially damaged, $\mathcal{D}_{p d}$, data sets as an indicator of the probability of structural damage. In the development of the damage measure which follows, the updated PDFs will be assumed to have unique maxima with respect to the model class, $\mathcal{M}_{N_{d}}$. The structural model class in this case is termed globally system identifiable. A further case in which there are a finite number of maxima of the updated PDF is not considered in this work. The structural model class for this second case is referred to as being locally system identifiable. When an infinite number of maximizing solutions exist, $\mathcal{M}_{N_{d}}$ is unidentifiable. This terminology is borrowed from Beck and Katafygiotis (1992).

The question might arise as to why the information given by the marginal distributions should be used to determine a measure of the probability of damage. The answer is simple. Each marginal distribution derived from an updated PDF contains information about the variation of a single parameter 
without assuming anything concerning the damage state of the remaining parameters. A precursor to the SHM method presented here had the limitation that damage was assumed to have occurred in only one substructure, or not at all (Beck, Vanik, and Katafygiotis 1994). This restriction has been lifted through use of the marginal distributions. As will be demonstrated, this leads to a very elegant way to address the general problem of damage in multiple substructures.

Prior to determining the marginal distributions and defining the damage measure, the relevant assumptions on the operating conditions and terminology for the joint PDFs are outlined. A few general observations on the behavior of the marginal PDFs are then made. Next, the damage measure is defined and its application to finding the existence and location of damage are discussed. Finally, some comments are made on the capability of the defined SHM strategy to determine the degree of damage.

\subsubsection{The Undamaged and Potentially Damaged PDFs}

The SHM method which is described in this work assumes that the structure under consideration for health monitoring is instrumented and that sets of modal parameters can be determined from measured data. Further, the assumption is made that the capability exists for the modal parameters to be determined on a time scale of the order of a few hours or better. Ambient vibration monitoring, described in Section 1.3, is a method which has the potential to meet this requirement. The framework can also be used in the case when measurements are taken on an irregular basis, such as when monthly or yearly inspections of the structure are made. However, this latter application does not address the problem of creating an automated monitoring system which is a focus of this work, so it is not treated.

After the structure is initially instrumented, and before the health mon- 
itoring can begin, an initial series of undamaged modal data sets are found from measured data. The undamaged measured modal parameter sets are referred to collectively by $\mathcal{D}_{u d}$. The assumption is made that the structure does not experience any significant changes during this initialization phase so that the measured sets well characterize the undamaged state of the structure.

The undamaged PDF, $p^{u d}\left(\theta \mid \mathcal{D}_{u d}\right)$, is defined as the updated PDF derived using a sufficient number of measured modal parameter sets from $\mathcal{D}_{u d}$ to achieve "stability" of the PDF. Thus

$$
p^{u d}\left(\theta \mid \mathcal{D}_{u d}\right)=p\left(\theta \mid \mathcal{D}_{u d}\right)
$$

where $p\left(\theta \mid \mathcal{D}_{u d}\right)$ is given by (2.72) In this work, the "stability" of the PDF is measured by how much the most probable model values vary as measured data sets are added. For the simulated data testing examples in Chapter 3 the number of measured modal parameter sets required for stability is determined by plotting the most probable parameters as more data is added, and observing when the change over a few cycles of added data does not considerably vary the parameters. This criterion is extremely subjective and ad hoc, but it appears to lead to good results in the simulation testing. Investigating a more objective measure of the stability is a possible topic for future work.

In the development which follows, a damage measure is defined which measures variations with respect to this PDF. During the initialization phase, the measured modal parameter sets are also used to form $p^{u d}\left(\theta \mid \mathcal{D}_{u d}\right)$ are used to determine how the damage measure varies due to noise when no damage is present in the structure. This characterization will enable bounds on the damage measure to be established.

Once the initialization phase is completed, the monitoring phase begins. In this phase, measured modal data is used by the SHM algorithm to monitor the damage state of the structure. Each measurement is the start of a new 
monitoring cycle. The monitoring time, $t_{m o n}$, is a discrete parameter which indicates the total number of monitoring cycles. The measured modal parameter sets for the monitoring phase constitute the potentially damaged data, $\mathcal{D}_{p d}$. The term "potentially damaged" is meant to infer that the state of the structure is unknown, not that it has necessarily been subjected to some damaging event. While $\mathcal{D}_{u d}$ is a static set, $\mathcal{D}_{p d}$ changes every time the structure is monitored. The number of potentially damaged data sets available is equal to $t_{\text {mon }}$. The $\mathrm{n}^{\text {th }}$ measured modal data set in $\mathcal{D}_{p d}$ is referred to by $\hat{\Upsilon}_{n} \cdot{ }^{3}$ As a notational convenience, the set $\mathcal{D}_{p d}$ is ordered such that the most recently measured modal data sets have the lowest indices. Thus, for a given $t_{m o n}, \hat{\Upsilon}_{1}$ is the newest modal parameter set. The measured set $\hat{\Upsilon}_{t_{m o n}}$ is always the oldest measured set.

During a given monitoring cycle, let the potentially damaged PDFs be the sequence $\left\{p_{k}^{p d}\left(\theta \mid \mathcal{D}_{p d}, t_{m o n}\right): k \in\left\{N_{\text {win }}^{\text {min }}, \ldots, N_{\text {win }}^{\max }\right\}\right\}$, where each $p_{k}^{p d}$ is calculated using only the modal data from the $k$ most recent monitoring cycles. The potentially damaged PDFs are therefore

$$
\begin{aligned}
p_{k}^{p d}\left(\theta \mid \mathcal{D}_{p d}, t_{m o n}\right) & =p\left(\theta \mid \hat{\Upsilon}_{1}, \hat{\Upsilon}_{2}, \ldots, \hat{\Upsilon}_{k}\right) \\
& =c_{p d} p(\theta) p\left(\hat{\Upsilon}_{1}, \hat{\Upsilon}_{2}, \ldots, \hat{\Upsilon}_{k} \mid \theta\right)
\end{aligned}
$$

where $c_{p d}$ is a normalizing constant. The sequence of subsets $\left\{\mathcal{D}_{k}\right\}$ of $\mathcal{D}_{p d}$, associated with these PDFs is

$$
\mathcal{D}_{k} \equiv\left\{\hat{\Upsilon}_{1}, \hat{\Upsilon}_{2}, \ldots, \hat{\Upsilon}_{k}\right\} \subset \mathcal{D}_{p d}
$$

\footnotetext{
${ }^{3}$ Similar terminology is not necessary for the elements of $\mathcal{D}_{u d}$ as they are never explicitly referenced.
} 
Each subset has a selection function $s_{k}$ such that

$$
s_{k}(n)=\left\{\begin{array}{ll}
1 & \text { if } n \leq k \\
0 & \text { otherwise }
\end{array} .\right.
$$

Using the assumption discussed in Section 2.4 that the PDFs for the different data sets are independent, $p_{k}^{p d}$ can be further written as

$$
p_{k}^{p d}\left(\theta \mid \mathcal{D}_{p d}, t_{m o n}\right)=c_{p d} p(\theta) \prod_{j=1}^{k} p\left(\hat{\Upsilon}_{j} \mid \theta\right)
$$

For each $k, p_{k}^{p d}$ is simply the product of previous PDF in the sequence, $p_{k-1}^{p d}$, and the PDF for the data set from the $\mathrm{k}^{\text {th }}$ prior monitoring cycle, $p\left(\hat{\Upsilon}_{k} \mid \theta\right)$. The parameter $k$ is referred to as the monitoring cycle window length or monitoring cycle window since it indicates how many data sets from past monitoring cycles are used in calculating $p_{k}^{p d}\left(\theta \mid \mathcal{D}_{p d}, t_{m o n}\right)$. The parameter $N_{w i n}^{m i n}$ determines the minimum number of data sets used to form the potentially damaged PDF. It will usually be set to 1 . The parameter $N_{w i n}^{\text {max }}$ defines the upper limit on how many monitoring cycles worth of measured data sets are selected to create the sequence of potentially damaged PDFs.

The damage measure, defined in a moment, uses changes between the potentially damaged PDFs and the undamaged PDF to monitor the state of the structure. The sequence of potentially damaged PDFs is used because taking only one subset of data from $\mathcal{D}_{p d}$ and forming a PDF to be compared to the undamaged PDF leads to problems. With one measured data set, the resulting PDF would be sensitive to variations due to damage and damage could theoretically be detected as soon as it occurred. The problem is that the PDF would also be sensitive to noise. Thus, damage may be indicated when there is none. Consider another case wherein all the data in $\mathcal{D}_{p d}$ is used to form the PDF for comparison. If $t_{m o n}$ is sufficiently large, then the effects 
of noise will be mitigated by the action of many data samples. Thus, false indications of damage will be reduced. Also, if the structure is damaged, and many samples of damaged modal parameters are in $\mathcal{D}_{p d}$, then smaller levels of damage will be detectable. Unfortunately, if damage occurs, the PDF will be strongly biased by the undamaged data already in $\mathcal{D}_{p d}$ so many monitoring cycles may pass before the damage is detected. In between these extremes are choices which trade-off between sensitivity, noise mitigation, and bias. Rather than consider only one such choice, the range of possibilities defined by $N_{w i n}^{m i n}$ and $N_{w i n}^{\max }$ are used. The lower limit $N_{w i n}^{m i n}$, is chosen large enough so that the noise effects are reduced to an acceptable level, but not so large as to sacrifice sensitivity. In theory, $N_{w i n}^{\max }$ could be chosen as large as $t_{m o n}$, in which case all the data in $\mathcal{D}_{p d}$ would be used in forming the last PDF in the sequence, $\left\{p_{k}^{p d}\right\}$. Practical limitations, such as computing time, prevent the use of such a large bound. In practice, therefore, the selection of $N_{\text {win }}^{\max }$ is made based on a tradeoff between increased sensitivity to small levels of damage and computation time.

\subsubsection{Observations on the Marginal Distributions}

The undamaged marginal PDF and cumulative distribution function (CDF) for the $\mathrm{i}^{\text {th }}$ structural model parameter are $\tilde{p}_{i}^{u d}\left(\theta_{i} \mid \mathcal{D}_{u d}\right)$ and $C_{i}^{u d}\left(\theta_{i} \mid \mathcal{D}_{u d}\right)$. These are derived by integrating the updated PDF $p^{u d}\left(\theta \mid \mathcal{D}_{u d}\right)$ in (2.74). The potentially damaged marginal PDFs and CDFs for the $\mathrm{i}^{\text {th }}$ structural model parameter are $\tilde{p}_{k i}^{p d}\left(\theta_{i} \mid \mathcal{D}_{p d}, t_{m o n}\right)$ and $C_{k i}^{p d}\left(\theta_{i} \mid \mathcal{D}_{p d}, t_{m o n}\right)$. These are derived by integrating the updated PDF $p_{k}^{p d}\left(\theta \mid \mathcal{D}_{p d}, t_{m o n}\right)$ in (2.75). In the discussion which follows, the arguments of the functions and the subscript " $i$ " will be dropped in order to simplify the notation. Keep in mind that the potentially damaged marginal quantities all depend on the monitoring time, so they vary as new data is acquired. Representative PDFs for $\tilde{p}^{u d}$ and $\tilde{p}_{k}^{p d}$ are shown in Figures 2.4 and 2.5. 
These PDFs have been scaled to both have the same maximum value. Since $\tilde{p}^{u d}$ is formed using many data sets, it will tend to be more peaked than $\tilde{p}_{k}^{p d}$, unless $k$ approaches the number of data sets used to form $\tilde{p}^{u d}$. For the case in Figure 2.4, $\tilde{p}_{k}^{p d}$ is said to be to the "left" of $\tilde{p}^{u d}$. Figure 2.4, shows a $\tilde{p}_{k}^{p d}$ which is to the "right" of $\tilde{p}^{u d}$.

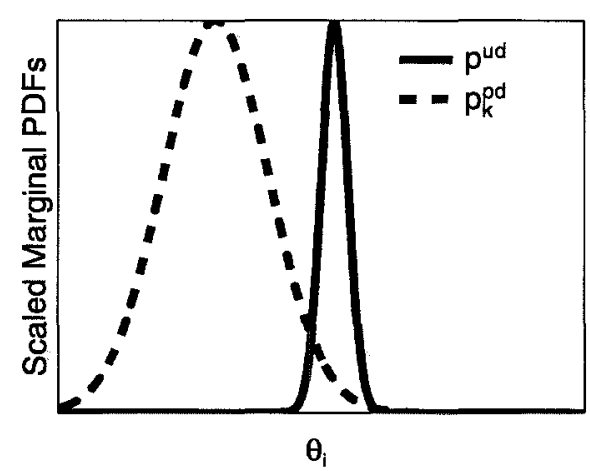

Figure 2.4 General PDFs derived from different data sets: $\tilde{p}_{k}^{p d}$ left

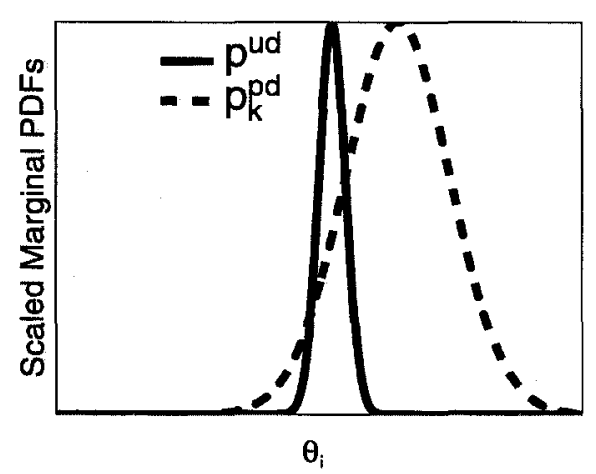

Figure 2.5 General PDFs derived from different data sets: $\tilde{p}_{k}^{p d}$ right

Some helpful qualitative observations can be made concerning the relationship between $\tilde{p}^{u d}$ and $\tilde{p}_{k}^{p d}$ using (2.78) and the plots in Figures 2.4 and 2.5. These observations will be used to qualitatively discuss the behavior of the damage measure defined in the next section.

The result (2.78) indicates that the sequence of joint distributions $p_{k}^{p d}$ are related through the product of PDFs derived using individual data sets, $p\left(\hat{\Upsilon}_{j} \mid \theta\right)$. For the purpose of illustrating the behavior of the marginal distribution $\tilde{p}_{k}^{p d}$ as $k$ and $t_{m o n}$ vary, the same relationship will be assumed to hold between the marginal distributions. Thus, $\tilde{p}_{k}^{p d}$ is treated as being related to $\tilde{p}_{k-1}^{p d}$ by taking the product $\tilde{p}_{k-1}^{p d} \tilde{p}^{\hat{\Upsilon}_{k}}$, where $\tilde{p}^{\hat{\Upsilon}_{k}}$ is the marginal PDF on $\theta_{i}$ derived from $p\left(\hat{\Upsilon}_{k} \mid \theta\right)$. Strictly speaking, this assumption is not true. However, for the qualitative nature of the observations which will be made, the 
assumption is fine.

In the absence of damage in the structure, $\tilde{p}^{\hat{\Upsilon}_{1}}$ will vary as $t_{\text {mon }}$ changes due to noise in the data. The PDF $\tilde{p}^{u d}$ is assumed to have been formed using a sufficient number of undamaged PDFs to be the "long-time" or "stabilized" PDF. Thus, new $\tilde{p}^{\hat{\Upsilon}_{1}}$ should be to the left of $\tilde{p}^{u d}$ as often as they are to the right if the structure is undamaged. This means that $\tilde{p}_{1}^{p d}$ should also be to the left of $\tilde{p}^{u d}$ as often as it is to the right as $t_{m o n}$ increases. The PDF $\tilde{p}^{u d}$ can be viewed as a "mean" PDF for the "distribution" of PDFs which characterize the variation in $\tilde{p}_{1}^{p d}$ due to noisy data.

As $k$ increases for a fixed $t_{m o n}$, more $\tilde{p}^{\hat{\Upsilon}_{j}}$ are multiplied together to form $\tilde{p}_{k}^{p d}$. For instance, $\tilde{p}_{2}^{p d} \sim \tilde{p}^{\hat{\Upsilon}_{1}} \tilde{p}^{\hat{\Upsilon}_{2}}$. Recall that $\tilde{p}^{\hat{\Upsilon}_{2}}$ for the current monitoring cycle, $t_{m o n}=t$, was $\tilde{p}^{\hat{\Upsilon}_{1}}$ in the last monitoring cycle, $t_{m o n}=t-1$. Therefore, variations at $k=1$ are propagated down the sequence of potentially damaged PDFs as $t_{m o n}$ increases.

Based on the observations in the preceding paragraphs, the behavior of the sequence $\tilde{p}_{k}^{p d}$ can be viewed as a random walk about $\tilde{p}^{u d}$. However, as $k$ increases, $\tilde{p}_{k}^{p d}$ stabilizes, so the magnitude of the variation attenuates. The most variation should therefore be observed for small values of $k$. These observations are used when the bounds on the damage measure are discussed.

\subsubsection{Defining the Probability of Variation}

The metric used to measure the difference between the undamaged and potentially damaged PDFs is now introduced. Since the the model class is assumed to be globally system identifiable, the updated PDFs will have unique maxima with respect to the structural parameters. Recall that the structural model parameters which give the maximum for a given PDF, $\hat{\theta}$, can be viewed as the most probable models given the data.

Let $\hat{\theta}^{u d}$ be the most probable model for $p^{u d}$. Take $\hat{\theta}_{k}^{p d}$ to be the most 
probable model for $p_{k}^{p d}$. In a deterministic setting, the components of $\hat{\theta}^{u d}$ and $\hat{\theta}_{k}^{p d}$ would be compared directly to look for changes in the structural model parameters based on the most recently measured data. As has been noted repeatedly, the uncertainties in the model parameters can lead to some difficulty in judging whether or not the structure is damaged based on such a direct comparison of individual models from the model class.

A better approach, and the one taken in this study, is to ask for each substructure, "What is the probability that $\theta_{k i}^{p d}<\theta_{i}^{u d}$, where the marginal PDFs for the parameters, $\tilde{p}_{i}^{u d}$ and $\tilde{p}_{k i}^{p d}$, are found using the undamaged data, $\mathcal{D}_{u d}$, and the subset $\mathcal{D}_{k}$ of the currently available measured modal data, $\mathcal{D}_{p d}$, respectively?" This question is answered by solving for the probability of variation from the undamaged state, or simply probability of variation for each substructure:

$$
P_{i}^{v a r}\left(t_{m o n}, k\right) \equiv P\left\{\theta_{k i}^{p d}<\theta_{i}^{u d} \mid \mathcal{D}_{u d}, \mathcal{D}_{p d}\right\}
$$

Large values indicate that $\theta_{k i}^{p d}<\theta_{i}^{u d}$, and small values should indicate that $\theta_{k i}^{p d}>\theta_{i}^{u d}$. Thus, the measure can potentially be used to detect increases and decreases in the stiffness parameters of a model. Only the application to decreasing model parameters is considered for health monitoring purposes.

The $\left(t_{m o n}, k\right)$ appears in (2.79) to explicitly show the dependence on the monitoring time and the monitoring cycle window. For a given $k$, as $t_{\text {mon }}$ increases, new measured modal data sets are made available and different modal parameter sets are used to determine the PDF for $\theta_{k i}^{p d}$. With $t_{m o n}$ held fixed, $P_{i}^{v a r}\left(t_{m o n}, k\right)$ varies with $k$ since different amounts of modal data enter in forming the $p_{k}^{p d}$.

If $p\left(\theta_{k i}^{p d}, \theta_{i}^{u d} \mid \mathcal{D}_{u d}, \mathcal{D}_{p d}\right)$ is the joint distribution for $\theta_{k i}^{p d}$ and $\theta_{i}^{u d}$, then the 
probability of variation is found by solving ${ }^{4}$

$$
\iint_{D} p\left(\theta_{k i}^{p d}, \theta_{i}^{u d} \mid \mathcal{D}_{u d}, \mathcal{D}_{p d}\right) d \theta_{i}^{u d} d \theta_{k i}^{p d}
$$

where $D$ is the area $\theta_{k i}^{p d}<\theta_{i}^{u d}$. This area is depicted in Figure 2.6.

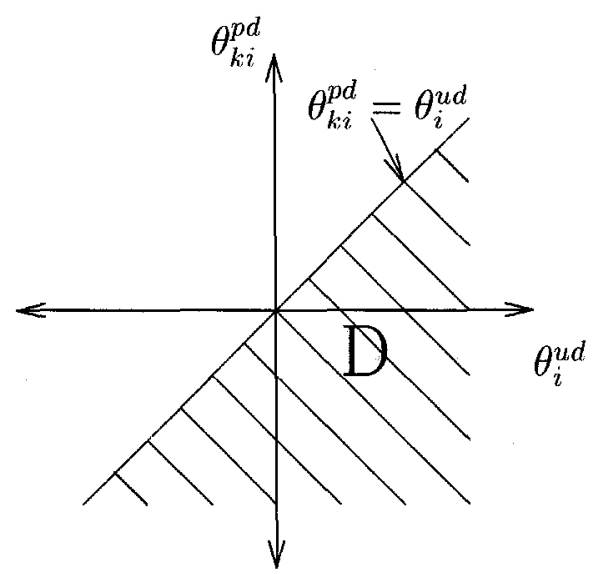

Figure 2.6 This plot shows the area, $D$, over which the joint PDF is integrated in order to determine $P\left\{\theta_{k i}^{p d}<\theta_{i}^{u d} \mid \mathcal{D}_{u d}, \mathcal{D}_{p d}\right\}$.

The $\theta_{i}^{u d}$ and $\theta_{k i}^{p d}$ are modeled as independently distributed, with marginal PDFs $\tilde{p}_{i}^{u d}$ and $\tilde{p}_{k i}^{p d}$ respectively. This simply reflects that knowing the undamaged parameters gives no information about the value of the potentially damaged parameters. The joint distribution for the parameters is then a product of the marginal PDFs:

$$
\tilde{p}\left(\theta_{k i}^{p d}, \theta_{i}^{u d} \mid \mathcal{D}_{u d}, \mathcal{D}_{p d}\right)=\tilde{p}_{i}^{u d}\left(\theta_{i}^{u d}\right) \tilde{p}_{k i}^{p d}\left(\theta_{k i}^{p d}\right)
$$

${ }^{4}$ Strictly speaking, the values of $\theta_{k i}^{p d}$ and $\theta_{i}^{u d}$ cannot be negative. In all practical applications, most of the probability volume of the joint distribution will lie where both $\theta_{i}^{u d}$ and $\theta_{k i}^{p d}$ are positive. Thus, the calculations are only performed using positive values of the parameters. Maintaining the infinite integral is a mathematical convenience which is used later to show that $P_{i}^{v a r}\left(t_{m o n}, k\right)=50 \%$ when $\tilde{p}_{i}^{u d}=\tilde{p}_{k i}^{p d}$. 
Using (2.81), the integral in (2.80) becomes

$$
\iint_{D} \tilde{p}_{i}^{u d}\left(\theta_{i}^{u d}\right) \tilde{p}_{k i}^{p d}\left(\theta_{k i}^{p d}\right) d \theta_{i}^{u d} d \theta_{k i}^{p d}
$$

The parameter $\theta_{i}^{u d}$ is integrated from $\theta_{k i}^{p d}$ to $\infty$, giving

$$
P_{i}^{v a r}\left(t_{m o n}, k\right)=\int_{-\infty}^{\infty}\left(1-C_{i}^{u d}\left(\theta_{k i}^{p d}\right)\right) \tilde{p}_{k i}^{p d}\left(\theta_{k i}^{p d}\right) d \theta_{k i}^{p d}
$$

Equivalently, $\theta_{k i}^{p d}$ could be integrated from $-\infty$ to $\theta_{i}^{u d}$, giving

$$
P_{i}^{v a r}\left(t_{m o n}, k\right)=\int_{-\infty}^{\infty} C_{k i}^{p d}\left(\theta_{k i}^{u d}\right) \tilde{p}_{i}^{u d}\left(\theta_{i}^{u d}\right) d \theta_{i}^{u d}
$$

\subsubsection{Considerations in the Use of $P^{v a r}$}

In order to determine the practical possibilities for use of this measure, the change in $P_{i}^{v a r}\left(t_{m o n}, k\right)$ under different levels of damage and the variation due to noise and modeling error when there is no damage must be characterized ${ }^{5}$. If the variation from parameter uncertainty effects cause $P^{v a r}$ to fluctuate from $0 \%$ to $100 \%$, or the change in $P^{v a r}$ for different degrees of damage is extremely small, then $P^{v a r}$ may not be a useful measure for health monitoring. If, however, tight bounds on the variation when no damage is present could be set and $P^{v a r}$ were very sensitive to damage, $P^{v a r}$ would be a practically useful indicator of damage. Experience using $P^{v a r}$ with simulated data has shown that reality lies between these extremes, and that $P^{v a r}$ can be used to detect damage.

The first step in using $P^{v a r}$ for health monitoring is establishing the likely fluctuations for each $k$ as the structure is monitored (i.e. $t_{m o n}$ increases) when no damage is present. Let $\tilde{p}_{k i}^{p d}=\tilde{p}_{i}^{u d}$. In this case, $P^{v a r}=50 \%$. This result is

\footnotetext{
${ }^{5}$ The subscript and $\left(t_{m o n}, k\right)$ are dropped in future references to the probability of variation in this discussion in order to simplify the notation and make the text more readable.
} 
easy to prove:

$$
\begin{aligned}
P_{i}^{v a r}\left(t_{m o n}, k\right) & =\int_{-\infty}^{\infty}\left(1-C_{i}^{u d}\left(\theta_{k i}^{p d}\right)\right) \tilde{p}_{k i}^{p d}\left(\theta_{k i}^{p d}\right) d \theta_{k i}^{p d} \\
& =\int_{-\infty}^{\infty}\left(1-C_{i}^{u d}\left(\theta_{k i}^{p d}\right)\right) \tilde{p}_{i}^{u d}\left(\theta_{k i}^{p d}\right) d \theta_{k i}^{p d} \\
& =\int_{-\infty}^{\infty} \tilde{p}_{i}^{u d}\left(\theta_{k i}^{p d}\right) d \theta_{k i}^{p d}-\int_{-\infty}^{\infty} C_{i}^{u d}\left(\theta_{k i}^{p d}\right) \tilde{p}_{i}^{u d}\left(\theta_{k i}^{p d}\right) d \theta_{k i}^{p d} \\
& =1-\frac{1}{2} \int_{-\infty}^{\infty} \frac{d\left(\left(C_{i}^{u d}\left(\theta_{k i}^{p d}\right)\right)^{2}\right.}{d \theta_{k i}^{p d}} d \theta_{k i}^{p d} \\
& =1-\frac{1}{2}\left[\left(C_{i}^{u d}\left(\theta_{k i}^{p d}\right)\right)^{2}\right]_{-\infty}^{\infty} \\
& =\frac{1}{2} .
\end{aligned}
$$

Noise in the data will cause $\tilde{p}_{k i}^{p d}$ to vary without any damage in the structure. Thus, $\tilde{p}_{k i}^{p d} \neq \tilde{p}_{i}^{u d}$ in general. This will lead to fluctuations in $P^{v a r}$ as the structure is monitored, even when damage is not present. The fluctuations will have the same behavior as fluctuations in $\tilde{p}_{k i}^{p d}$ relative to $\tilde{p}_{i}^{u d}$. Thus, $P_{i}^{v a r}\left(t_{m o n}, k\right)$ will be expected to look like a random walk about $50 \%$ which attenuates as $k$ increases. Also, changes introduced in $P_{i}^{v a r}\left(t_{m o n}, k\right)$ when $k=1$ and $t_{m o n}=N$ will affect $P_{i}^{v a r}\left(t_{m o n}, k\right)$ at $k=1+M$ when $t_{m o n}=N+M$.

If limits on the fluctuations could be established, exceeding the limits for any $k$ as $t_{m o n}$ increases would give a possible indication of damage. Since extreme variations of $P_{i}^{v a r}\left(t_{m o n}, k\right)$ due to "random" extreme data sets are always possible, the limits would necessarily be soft, rather than hard. Thus, they should be found using probability theory. In order to determine limits in an analytical fashion, a likely range of variation for $P_{i}^{v a r}\left(t_{m o n}, k\right)$ as a function of the noise in the data would need to be established through another level of probability modeling.

This probabilistically rigorous and conceptually challenging analytical approach is left for future work. Instead, the limits are established empirically in 
any given application based on the behavior of $P_{i}^{v a r}\left(t_{m o n}, k\right)$ as the structure is monitored during the initialization phase. The mean value of $P_{i}^{v a r}\left(t_{m o n}, k\right)$ for a fixed $k$ is $\bar{P}_{i}^{d a m}(k)$. The standard deviation is $\sigma_{P_{i}^{v a r}}(k)$. Using these statistics, a sequence of limits, $P_{i}^{\text {alarm }}(k)$, is chosen to be

$$
P_{i}^{\text {alarm }}(k)=\bar{P}_{i}^{\text {dam }}(k)+\gamma \sigma_{P_{i}^{v a r}}(k) .
$$

If $P_{i}^{\text {alarm }}(k)$ exceeds $100 \%$ form some value of $k$, then it is set to be $99 \%$ for that value of $k$. This ad hoc fix is implemented so that damage still has the possibly of being detected.

The parameter $\gamma$ determines the relative frequency of indicating damage when there is none (i.e. a false alarm) and missing damage when it exists (i.e. a missed alarm). For instance, suppose $P_{i}^{\text {var }}\left(t_{m o n}, k\right)$ could be accurately modeled as being Gaussian distributed for each $k$, with the means and standard deviations $\bar{P}_{i}^{\text {dam }}(k)$ and $\sigma_{P_{i}^{v a r}}(k)$. For a choice of $\gamma=1$ then, $P_{i}^{\text {var }}\left(t_{m o n}, k\right)$ will exceed $P_{i}^{a l a r m}(k)$ about $17 \%$ of the time. For $\gamma$ set to 3 , the chance of exceeding the bounds should fall to less than $1 \%$. Thus $\gamma$ should be set as high as possible in order to avoid false alarms. The problem with doing so is that the possibility of missing a damage event increases as $\gamma$ is increased. Choosing $\gamma$ to reduce missed alarms increases the possibility of false alarms. The effects of different choices of $\gamma$ are discussed for the 2-DOF simulation example in Section 3.2. The results of this discussion are used to set $\gamma$ in the 10-DOF example in Section 3.3.

\subsubsection{Sounding an Alarm}

During a given monitoring cycle, if $P_{i}^{\text {var }}\left(t_{m o n}, k\right)$ exceeds $P_{i}^{\text {alarm }}(k)$ for any $k$, an alarm that the substructure for which the excessive variation occurred may 
be "damaged" is sounded. Thus,

$$
\begin{aligned}
P_{i}^{\text {var }}\left(t_{m o n}, k\right) & \geq P_{i}^{\text {alarm }}(k) \text { For Any } k \Rightarrow \text { "Damage Alarm" } \\
& <P_{i}^{\text {alarm }}(k) \text { For All } k \Rightarrow \text { "No Damage." }
\end{aligned}
$$

Recall that $P_{i}^{\text {alarm }}$ is a soft level rather that a hard level. Hence, an additional level of decision making must be added when the damage alarm is set in order to determine whether the level was exceeded due to damage, or an extreme data set. In addition to the damage criterion in (2.87), therefore, other criteria must be considered in order to determine the difference between false and true alarms. In practice with simulated data, $P_{i}^{v a r}\left(t_{m o n}, k\right)$ is found to have characteristic behaviors depending on whether the structure is undamaged or damaged. Therefore, the behavior of $P_{i}^{v a r}\left(t_{m o n}, k\right)$ can be studied by a human or an expert system as a function of $t_{m o n}$ and $k$ in order to determine the state of damage. During the description of the simulation testing in Chapter 3, the behaviors of $P_{i}^{v a r}\left(t_{m o n}, k\right)$ for a simple structure and some guidelines for use in deterimining a true state of damage from a false one are discussed.

\subsubsection{Applying $P^{v a r}$ for SHM}

The relationships defined in (2.83) and (2.87), together with $P_{i}^{\text {alarm }}(k)$ in (2.86) give a measure for damage in a substructure, and a criterion by which to use that measure to determine the potential existence of damage. By applying the procedure to each of the structural model parameters in turn, $P_{i}^{\text {var }}\left(t_{\text {mon }}, k\right)$, the probability of variation for the $\mathrm{i}^{\text {th }}$ substructure, can be found. The parameters for which the probability of variation exceeds $P_{i}^{\text {alarm }}(k)$, the $\mathrm{i}^{\text {th }}$ alarm function, are called the damaged parameters. The remaining parameters are undamaged parameters. As mentioned in Section 2.4.2, stiffness variations in the model substructures are considered proxies for the variations in the sections of the 
structure modeled by the substructures. Therefore, $P_{i}^{v a r}\left(t_{m o n}, k\right)$, is also taken as the probability of variation in the structure in the section corresponding to the $i^{\text {th }}$ structural parameter. Hence, the structural sections corresponding to the damaged parameters are considered damaged.

In practice, the SHM method would not be applied directly as has been described due to practical considerations. Acquiring data could be costly. Also, identifying the modal parameters from the measured data may take some time. Thus, the rate at which data is taken is constrained and always finding measured modal parameters in the shortest time intervals possible may not be economically desirable. Finally, calculating every potentially damaged PDF in the sequence $\left\{p_{k}^{p d}\right\}$ for a wide enough range of $k$ to take advantage of the sensitivity for low $k$ and noise reduction for high $k$ may not be computationally feasible. Different procedures which take these factors into account can be established.

As an example, consider the following procedure. Let many sets of undamaged data be available and assume that the $P_{i}^{\text {alarm }}(k)$ have already been established. Let the modal parameters from a structure under consideration only be regularly monitored every eight hours or when triggered by a severe loading event. Suppose, however, that the monitoring system is capable of collecting data every fifteen minutes. Rather than evaluate every potentially damage PDF in the sequence, only calculate it at enough points to capture the whole range. Thus, $P_{i}^{v a r}\left(t_{m o n}, k\right)$ would be calculated at only a few points in the range of interest. If the damage alarm is not triggered when a single set of the regularly monitored or load-triggered data is added, then no action is taken. If the damage alarm criterion is satisfied for some substructures, but relevant $P_{i}^{\text {var }}\left(t_{m o n}, k\right)$ exceed $P_{i}^{a l a r m}(k)$ at only a few $k$ or by not very much, rather than sound an immediate alarm, increase the rate at which measured data is found. Should the new data increase the degree to which 
the $P_{i}^{v a r}\left(t_{m o n}, k\right)$ are beyond the alarm functions, damage would officially be indicated. If the additional data reduces the probabilities of variation which triggered the alarm so that they fall below the corresponding limits, then consider the alarm a false one. This logic could be implemented in an expert system which manages the SHM method.

Other possibilities exist for implementation methods. These operational issues for applying the SHM method are left for future work, since they will depend significantly on the application under consideration.

The proposed SHM method provides a way to establish the existence and location of damage in a structure. Furthermore, the ill-conditioning in the problem is taken into account through the use of the PDFs to include the uncertainty in the measured data and the modeling error in determining the probability of damage. The remaining issue is determining the degree of damage in a substructure once an alarm is set.

\subsubsection{Degree of Damage}

Once the existence and location of damage have been found, the final step in SHM is determining the degree of damage. Assume that a positive indication of damage has been detected through use of the method just described. Group the data which corresponds to the damaged state into $\mathcal{D}_{d}$. The most probable damaged model, $\hat{\theta}_{d}$, is computed using only $\mathcal{D}_{d}$, so that the other data does not bias the identified model. Let the measure of the degree of damage or simply the degree of damage for $\theta_{i}$ be

$$
\Delta \theta_{i}=\frac{\hat{\theta}_{i}^{u d}-\hat{\theta}_{i}^{d}}{\hat{\theta}_{i}^{u d}}
$$

If the substructure is itself an element, then the measure will indicate change in that element. However, a substructure will generally consist of many elements, 
so this measure will reflect the damage of elements of a substructure "averaged" over an entire substructure. Interpreting what the degree of damage indicates is then a function of the substructure. For a very simple substructure consisting of only a few members or elements, a $10 \%$ degree of damage might not indicate much change in the individual elements. However, an equivalent change in a substructure which contains many elements might represent a significant level of damage in a number of the elements. In the examples in Chapter 3, the degree of damage is compared to the known damage when damage is detected and located. However, an extensive study investigating the relationship between elemental damage and the degree of damage measure for substructure is left for future work.

\subsubsection{Calculating the Cumulative Distributions}

One final step remains before the Bayesian probabilistic SHM method defined in this section can be applied: the marginal PDFs and CDFs for the parameters must be found. Given an updated PDF for all the structural model parameters, $p(\theta \mid \mathcal{D})$ formed using data $\mathcal{D}$, the conditional CDF for the $\mathrm{i}^{\text {th }}$ structural model parameter, $C_{i}\left(\theta_{i}\right)$, is found by integrating the updated PDF over the entire possible space for all parameters except for the $\mathrm{i}^{\text {th }}$ structural model parameter. This results in

$$
C_{i}\left(\theta_{i}\right)=P\left(\tilde{\theta}_{i} \in\left[0, \theta_{i}\right] \mid \mathcal{D}\right)=\int_{\Theta_{i}} p(\tilde{\theta} \mid \mathcal{D}) d \tilde{\theta}
$$

where $\Theta_{i}$ is the set where all the $\tilde{\theta}$ vary across their full range, except $\tilde{\theta}_{i}$, which varies from 0 to $\theta_{i}$.

This integral cannot be evaluated analytically for anything more than a one-DOF problem. Numerical integration is feasible only for problems of very small dimension. Therefore, the value of the integral must be approximated 
in some other manner. Two procedures are suggested in this study for approximating the value of the integral. One is easy to calculate, but gives a poor approximation when the uncertainty is large. The other takes more time to compute, but is a better approximation. Both methods are based on a Laplace's method (Bleistein and Handelsman 1986) of asymptotic expansion for evaluating integrals.

\section{Coarse Asymptotic Expansion of the CDF Integral}

Because of its form, the updated PDF (2.72) lends itself well to approximation by a multivariable joint Gaussian distribution function. Let $\hat{\theta}$ maximize the updated PDF, and assume that only one maximum exists. Approximate $\tilde{J}(\theta)$ about $\hat{\theta}$, by

$$
\tilde{J}(\theta) \approx \tilde{J}(\hat{\theta})+\frac{1}{2}(\theta-\hat{\theta})^{T} L_{\theta}(\hat{\theta})(\theta-\hat{\theta})
$$

where $L_{\theta}(\hat{\theta})$ is the Hessian matrix of $\tilde{J}(\theta)$ with respect to $\theta$ evaluated at $\hat{\theta}$. The form of the updated PDF (2.72) reveals that using (2.90) in (2.72) will produce a Gaussian approximation for the updated PDF.

Substituting (2.72) and (2.90) into (2.89) leads to

$$
C_{i}\left(\theta_{i}\right)=\tilde{k} \int_{\Theta_{i}} \exp \left[-\frac{1}{2}\left(\tilde{J}(\hat{\theta})+\frac{1}{2}(\tilde{\theta}-\hat{\theta})^{T} L_{\theta}(\hat{\theta})(\tilde{\theta}-\hat{\theta})\right)\right] d \tilde{\theta}
$$

Integrals of this type occur often in reliability problems (Madsen, Krenk, and Lind 1986). In terms of the terminology from reliability theory, the boundary of the region defined by $\Theta_{i}$ defines a failure surface. The failure surface for this problem can be viewed as a hyperplane. In this case, the solution of the integral is very simple to express (Madsen, Krenk, and Lind 1986):

$$
C_{i}\left(\theta_{i}\right)=P\left(\tilde{\theta}_{i} \in\left[0, \theta_{i}\right]\right)=\Phi\left(\rho_{i}\left(\theta_{i}\right)\right)-\Phi\left(\rho_{i}(0)\right)
$$


where $\Phi$ is the cumulative distribution function for a Normally distributed random variable of zero mean and unit variance, and

$$
\rho_{i}\left(\theta_{i}\right)=\frac{\theta_{i}-e_{i}^{T} \hat{\theta}}{\sqrt{e_{i}^{T} L_{\theta}(\hat{\theta})^{-1} e_{i}}}=\frac{\theta_{i}-\hat{\theta}_{i}}{\sqrt{e_{i}^{T} L_{\theta}(\hat{\theta})^{-1} e_{i}}} .
$$

This process can also give an approximation to the PDF for each $\theta_{i}$ by evaluating the normal Gaussian function of zero mean and unit variance at the calculated values of $\rho$.

Since this approach is based on approximating the original PDF about its maximum by a Gaussian distribution function, the results should only be good when the Hessian matrix has large eigenvalues. Increasing eigenvalues in the Hessian matrix correspond to smaller variances, and thus less uncertainty. For reference, this approach is the coarse approximation.

The approximation method just described is a specific example of a general method for asymptotic approximation of integrals described by Papadimitriou, Beck, and Katafygiotis (1995). In order to use this method, the maxima of the updated PDF with respect to the model parameters needs to be found. Further, the Hessian matrix of $\tilde{J}(\theta)$ at the maxima must be determined. Appendix $B$ presents the details of these evaluations.

\section{Fine Asymptotic Expansion of the CDF Integral}

As noted previously, the form of the updated PDF (2.72) lends itself well to approximation by a multivariable joint Gaussian distribution function. In the coarse approximation, the PDF was approximated about its maximum with respect to all of the parameters and integrated using a result from reliability theory. If improved accuracy were necessary, a small change could be made to the coarse method, resulting in the fine approximation. 
Consider the marginal PDF for the $\mathrm{i}^{\text {th }}$ model parameter.

$$
\tilde{p}\left(\theta_{i} \mid \mathcal{D}\right)=\int_{\Theta^{i}} p\left(\theta_{i}, \theta^{i} \mid \mathcal{D}\right) d \theta^{i}
$$

where $\theta^{i}=\left[\theta_{1}, \ldots, \theta_{i-1}, \theta_{i+1}, \ldots, \theta_{N_{\theta}}\right]^{T}$ and $\Theta^{i}$ is the set where $\theta^{i}$ varies across its full possible range. For a fixed $\theta_{i}$, this integral can be approximated using the previously described asymptotic approximation technique. By approximating the integral at different values of $\theta_{i}$, an approximation to the marginal PDF for $\theta_{i}$ is derived. This marginal PDF can be numerically integrated to find the CDF for $\theta_{i}$.

Difficulties such as missing the maxima and determining what size step to take can be easily addressed. First, find the maximum of the PDF with respect to all of the structural parameters. This serves as a starting point for the calculation of the marginal PDF for each of the structural parameters, and will ensure that the maxima are not missed. Also, if the PDF is well-behaved, the maxima for the value of $\theta_{i}$ at which the marginal PDF is to be evaluated will be near the maxima for the previous value of $\theta_{i}$. This improves the efficiency of finding the maximum in each step. An appropriate step size can be determined from the marginal PDF found using the coarse approximation. The standard deviation for the coarse marginal PDF will indicate a characteristic length of the problem, so that a proper initial choice can be made for the necessary step size. The step size can be reduced and the PDF recalculated until the desired level of convergence is achieved. The number of steps can be found by continuing to step until the area added by an additional point is a specified fraction of the total area.

Since the Gaussian distribution function approximation is performed for each point $\theta_{i}$ in the marginal PDF in this method, the results are better than for the coarse approximation. The cost for the improved performance is that forming the CDF for each $\theta_{i}$ requires that the PDF be maximized with respect 
to the remaining parameters for each desired point in the marginal PDF. This is a computationally expensive procedure.

For the results presented in this study, the Hessian matrix of the MOF always has large enough eigenvalues that the coarse approximation is sufficient for all calculations. Since a study validating the use of the asymptotic expansion in this case has already been completed by Papadimitriou, Beck, and Katafygiotis (1995), these efforts are not duplicated. The fine approximation is not needed for the application presented here, so exploration of its capabilities and an extensive comparison to the coarse approximation is left for future work. The coarse approximation is used without further comment.

\subsection{Summary of the SHM Method}

SHM technique has been developed in the Bayesian probabilistic framework. The methodology uses frequencies and incomplete modeshapes measured from a structure to infer the existence, location, and possible degree of damage in the structure. The model parameter uncertainties due to noise in the data and modeling error inherent in the SHM problem are taken into account. The method is summarized as follows.

A set of observable modal parameters which can be measured from the structure under consideration is determined. The observable modal parameters consist of $N_{m}$ frequencies, $\omega_{r}$, and $N_{m}$ generally incomplete modeshapes, $\psi_{r} \in \mathbb{R}^{N_{o}}$ determined from measurements on the structure. All the modal pa-

rameters from one test are called a set of measure modal data, $\hat{\Upsilon}$. A collection of modal data sets taken at different times is called data, $\mathcal{D}$. The data from a structure in a known undamaged state is undamaged data, $\mathcal{D}_{u d}$. Data from the same structure in an unknown state is considered potentially damaged data, $\mathcal{D}_{p d}$. All the data collected together is $\mathcal{D}_{N_{s}}$. 
One advantage afforded by using modal parameters is that ambient vibration testing can be employed to measure the necessary data. Ambient vibration testing is beneficial in a number of ways. First, for the low amplitude excitations typically experienced during ambient motion, most structural systems are well characterized with linear models, which are easier to treat than nonlinear ones. Also, the danger of further damaging the structure while testing it is reduced. Finally, continuous ambient vibration tests can be performed at very low cost. For a permanently instrumented structure, this will facilitate the implementation of the SHM method as an automatic monitoring system.

A set of deterministic models, $\mathcal{M}_{N_{d}}$, which have a dynamic behavior partly characterized by the equation of motion:

$$
M \ddot{x}+C(\theta) \dot{x}+K(\theta) x=f(t)
$$

with $f, x \in \mathbb{R}^{N_{d}}$, and $M, C, K \in \mathbb{R}^{N_{d} \times N_{d}}$ is introduced. The mass matrix is assumed known, and the damping matrix is assumed to be of a form so that the models possess classical normal modes. The models in $\mathcal{M}_{N_{d}}$ are defined by the structural parameters, $\theta \in \mathbb{R}^{N_{\theta}}$, and the expansion parameters, $\alpha=\left\{\alpha_{r} \in \mathbb{R}^{N_{d}}: r=1, \ldots, N_{m}\right\}$. The structural parameters parameterize $K(\theta)$ in terms of a linear combination of substructure stiffness matrices, $K_{i}$ :

$$
K(\theta)=K_{0}+\sum_{i=1}^{N_{\theta}} \theta_{i} K_{i}
$$

The substructure stiffness matrices model the contributions of a portion of the structure to the overall stiffness matrix. Expressing the dependence of the stiffness matrix on the structural parameters in this form is convenient for the mathematical analysis. However, the method which is developed does not preclude using a more general parameterization. For the purpose of health monitoring, the linear parameterization given is sufficient to enable determi- 
nation of the existence and location of damage. The expansion parameters specify linear combinations of the model modeshapes, $X(\theta)$, called expanded modeshapes:

$$
\phi_{r}\left(\theta, \alpha_{r}\right)=X(\theta) \alpha_{r}, \quad r \in 1, \ldots, N_{m},
$$

The model class is supposed to indicate what values the observable modal parameters will take. Due to noise in the measured data and modeling error, uncertainties are introduced. These uncertainties are characterized by introducing a class of probabilistic models, $\mathcal{P}$, in which the frequencies and modeshapes are assumed to be independently distributed from each other and from mode to mode. The Principle of Maximum Entropy is invoked to determine the form of the individual PDFs. Probability density functions (PDFs) for the observable parameters given a model in $\mathcal{M}_{N_{d}}$ are thus developed. In formulating the probability models, two parameters, $\sigma_{\omega_{r}^{2}}$ and $\epsilon_{\psi_{r}}$, are introduced. The proposed SHM method determines these parameters directly from the measured modal data. The final form of the PDF on the observable modal parameters contains one term corresponding to an eigenequation error, and another term which represents the modeshape error.

The observable data are then assumed measured so that $N_{s}$ measured modal data sets, $\left\{\hat{\Upsilon}_{1}, \ldots, \hat{\Upsilon}_{N_{s}}\right\} \equiv \mathcal{D}_{N_{s}}$ are available. Bayes' theorem is used to find the probability of the structural and expansion parameters given this measured data and the PDF on the observable data. In this process, a choice of the initial probability distribution for $\theta$ and $\alpha$ must be made. This initial probability distribution contains prior knowledge of the likely values of $\theta$ and $\alpha$. When all the necessary choices are made, the final form of the PDF for $\theta$ 
and $\alpha$ conditional on $\mathcal{D}_{N_{s}}$ is given as:

$$
p\left(\theta, \alpha \mid \mathcal{D}_{N_{s}}\right)=\tilde{k}(\epsilon, \sigma) \exp \left[-\frac{1}{2} J(\theta, \alpha)\right]
$$

where

$$
J(\theta, \alpha)=\left(\theta-\theta_{F}\right)^{T} S^{-1}\left(\theta-\theta_{F}\right)+\sum_{n=1}^{N_{m}} J_{r}\left(\theta, \alpha_{r}\right)
$$

and

$J_{r}\left(\theta, \alpha_{r}\right)=\sum_{n=1}^{N_{s}} s(n)\left[\frac{\left\|\left(K-\hat{\omega}_{r}^{2}(n) M\right) \phi_{r}\right\|_{M^{-1}}^{2}}{\sigma_{\omega_{r}^{2}}^{2}\left\|\phi_{r}\right\|_{M}^{2}}+\frac{\phi_{r}^{T} \Gamma^{T}\left(I-\psi_{r}(n) \psi_{r}^{T}(n)\right) \Gamma \phi_{r}}{\epsilon_{\psi_{r}}^{2}\left\|\Gamma \phi_{r}\right\|^{2}}\right]$.

The marginal distribution on $\theta$ is obtained by integrating this PDF over all the possible $\alpha$. Due to the complexity of the integrand, the integration is not done exactly. Instead, an asymptotic approximation is made to the integral. Since the PDF tends to be very peaked as a function of the $\alpha_{r}$ for a variety of different models, the approximation is very good. The resulting marginal $\mathrm{PDF}$ is

$$
p\left(\theta \mid \mathcal{D}_{N_{s}}\right)=\tilde{k} \exp \left[-\frac{1}{2} \tilde{J}(\theta)\right]
$$

where

$$
\tilde{J}(\theta)=J(\theta, \hat{\alpha}(\theta))
$$

and $\hat{\alpha}(\theta)$ is the value of $\alpha$ that maximizes $p\left(\theta, \alpha \mid \mathcal{D}_{N_{s}}\right)$ for a given $\theta$.

The marginal distribution on $\theta$ is further integrated over each of the parameters but one to get the marginal distribution for a single parameter, $\theta_{i}$. This process is continued for each parameter so that all the individual marginal 
distributions are found. As the integration cannot be done analytically, two approximation techniques are presented. One is a coarse approximation in which $p\left(\theta \mid \mathcal{D}_{N_{s}}\right)$ is approximated by a Gaussian distribution about its peak, and the marginal on $\theta_{i}$ is found using results from reliability theory. The other approximation is more exact. For each parameter, an asymptotic expansion solution for the integral over the other parameters is obtained at a series of discrete values of the non-integrated parameter. In this manner, a discrete marginal PDF is formed. This approximation is closer to the true distribution than the first approximation. The trade-off is that the second method takes much longer to compute. For the cases of interest in this work, the coarse approximation is sufficient to evaluate the integrals. Thus, the presented results use the coarse approximation.

A measure for the probability of variation of model parameters from a reference state, $P_{i}^{v a r}$, is defined. The damage measure is calculated based on the probability that the structural model parameters found using recently measured modal data are less than those parameters found using the reference, or undamaged set. If $P_{i}^{v a r}$ exceeds a certain level, $P_{i}^{a t e r t}$, an alert is raised that the structure may be damaged. Determination of whether the alert is a true indication of damage or a false alarm is handled by studying other aspects of $P_{i}^{v a r}$. Calculating the measure for each substructure and determining if the damage alarm has been set provides a way to determine the existence and location of damage. A possible measure of the degree of damage is also introduced. A manner in which to trade-off between the possibility of false and missed alarms is also discussed.

In practice, the SHM method could be applied as an online automated structural health monitoring system through the following procedure. This procedure assumes that the structure under consideration has already been instrumented, and the model class and probability models for the application 
have already been determined.

- During an initialization phase, take as many measurements from the structure in the undamaged state as are needed to find a stable reference undamaged $\mathrm{PDF}$ and reasonable bounds on the variations in $P_{i}^{\text {var }}$ for each substructure when there is no damage in the structure.

- Start the monitoring phase wherein the structure is measured periodically and $P_{i}^{v a r}$ in each substructure is calculated after each measurement.

- If $P_{i}^{v a r}$ does not exceed $P_{i}^{a l e r t}$, wait for the next set of data.

- If $P_{i}^{v a r}$ exceeds $P_{i}^{a l e r t}$, consult an expert system (human or computer encoded logic) about how to proceed. The expert system decides whether the alarm appears false, true, or uncertain based on criteria establish through previous investigations. If the alarm is determine to be true, the appropriate safety measures are implemented immediately. For false or uncertain alarms, the rate at which data is taken is increased and the additional data used to further determine the validity of the alarm.

The following chapter reports on the results of testing the method. Computer models of two structures, and synthetically generated data are used to explore the various features, strengths, and limitations of the SHM method. 


\section{Chapter 3}

\section{Simulated Data Testing}

\subsection{Introduction}

The SHM method developed in Chapter 2 is examined through testing using mathematical models of structures. Two models are considered: a 2-DOF shear structure in Section 3.2, and a 10-DOF shear structure in Section 3.3. The 2-DOF shear structure is a simple example which can be exhaustively explored and used to examine some fundamental behaviors of the SHM method. The 10-DOF case adds some of the problems that will be encountered in real applications such as limited modeshape information, high-dimensionality, and substructuring. Use of mathematical models enables the behavior of the SHM method to be analyzed in a controlled way.

In theory, the damage detection and automatic monitoring goals are satisfied by the technique presented in Chapter 2. However, the development of the SHM procedure in Chapter 2 followed an analytical and logical line of reasoning in which many assumptions were made. Testing of the method is a check on the legitimacy of these assumptions. The most critical claim of the SHM method developed in this paper is that the suggested damage measure can be used to determine the existence and location of damage in the presence of the noise and modeling errors. Establishing the validity of this claim is one 
focus of the testing in this chapter. As one of the intentions of this study was to develop a method that will lend itself well to automation, some guidelines for potential use by an expert system in interpreting the existence of damage are discussed.

\subsubsection{Common Definitions}

Before describing the examples in more detail, some aspects common to all of the examples are discussed.

\section{Noise in the Data}

For each model, the noisy measured modal data is generated by adding random values chosen from zero-mean Gaussian distributions to the true modal parameters of the model. The resulting data is referred to as synthetic data. The percent standard deviations (i.e. coefficients of variation) on the frequencies and modeshapes used in this study are shown in Table 3.1. In terms of the development in Chapter 2, the values in the table are $\epsilon_{\omega_{r}^{2}}$ and $\epsilon_{\psi_{r}}$. These

\begin{tabular}{|c|c|}
\hline \multicolumn{2}{|c|}{ Modal Parameter } \\
\hline$\omega^{2}$ & $\psi$ \\
\hline $2.0 \%$ & $2.0 \%$ \\
\hline
\end{tabular}

Table 3.1 The noise levels on the modal parameters

standard deviations represent expected standard deviations on the measured modal data for well-expressed modes based on the author's experience with measuring modal data from a laboratory structure and multiple full-scale structures (Beck et al. 1995).

In practice, the noise levels generally vary from mode to mode, and worsen for higher modes. Also, for a given application, the relationship between the 
frequency and modeshape noise levels may not be the same as designated in Table 3.1. Adding these levels of complexity in not considered necessary in this exploratory study.

Looking back to the modal measure of fit (2.65) and relations (2.50) to (2.54) for the probability model parameters reveals that level of noise in the modal parameters, as determined by $\epsilon_{\omega_{r}^{2}}$ and $\epsilon_{\psi_{r}}$, enters the MMOF in a reciprocal squared fashion. Data sets are included in the MMOF through a linear summation. Therefore, in theory, increasing $\epsilon_{\omega_{r}^{2}}$ or $\epsilon_{\psi_{r}}$ by a factor of two should be effectively offset by having four times as many data sets. Testing with simulated data confirmed this behavior. Based on this result, the analyses in this work are presented for only the single level of noise described in Table 3.1. The implied understanding is that results for different noise levels can be considered by appropriately scaling the number of measured modal data sets.

\section{Model Class}

The model class in each case uses the corresponding mathematical model as the fundamental model. This allowed for different possible substructure configurations, although for the 2-DOF model only two such configurations exist. In each example, the actual fundamental model and substructuring used to generate the model class are described in detail. The parameter values used in the underlying fundamental model are included so that results from future studies can be properly compared with this one.

\section{Initial Distribution for $\theta$}

For each case examined, the initial distribution on $\theta$ had to be selected. Recall that the initial distribution is a joint Gaussian distribution with mean given by $\theta_{F}$ and a diagonal covariance matrix $S((2.61)$ and (2.63)). In this study, all of 
the $\sigma_{i}$ in $S$ are assumed equal to the same value, $\sigma=0.5$. Figure 3.1 shows the initial distributions of one structural parameter for $\sigma=1.0, \sigma=0.5, \sigma=0.1$. The distributions for $\sigma=0.1$ and $\sigma=1.0$ demonstrate low uncertainty and

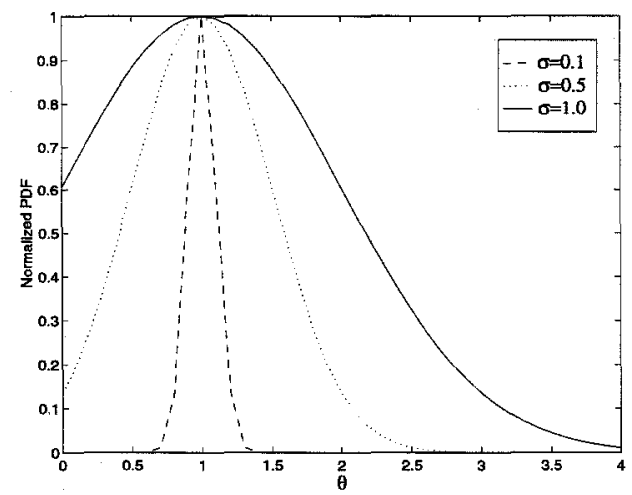

Figure 3.1 Three possible initial distributions for $\theta_{i}$, with $\sigma=0.1$, $\sigma=0.5$, and $\sigma=1.0$.

high uncertainty in the initial modeling. A choice of $\sigma=0.1$ hampers health monitoring effort for cases with few sets of data since too much weight is put on the fundamental model. If $\sigma=1.0$, the accepted possible variation in $\theta$ includes significant amounts of probability volume below zero and for values more than twice the fundamental model values. For these reasons, they were both unacceptable values of $\sigma$. The selection of $\sigma=0.5$ struck a balance between wanting to reflect some initial uncertainty so that the SHM method is not insensitive to damage, and maintaining reasonable bounds on the likely values for the structural parameters.

When the noise levels are high and few data sets are available, the initial distribution will have a significant impact on the updated distribution. However, as more data sets become available, the choice for the initial distribution becomes less important since the data will control the form of the updated distribution. Therefore, in all the results, only the distribution with $\sigma=0.5$ is 
considered and variation of the performance of the SHM with changing initial distribution is not explored.

\subsubsection{Brief Descriptions of the Examples}

For the 2-DOF example, the dependence of the procedure's performance on the alarm function, $P_{i}^{a l a r m}(k)$, the degree and location of damage, and the number of identified modes are characterized. The "performance" is analyzed in terms of the relative frequency of false alarms for undamaged substructures and the ability to detect damage when damage is present. This characterization demonstrates the typical behaviors of the method. Studying these behaviors provides guidelines for how the method should be implemented in other more realistic problems.

In the 10-DOF example, the guidelines suggested by the 2-DOF example are used to establish the alarm functions. The avenues of investigation in these case are limited by the size of the problems. Thus, only a few representative cases are examined. The locations and degrees of damage, as well as the amount of modal data are varied. These cases demonstrate some of the strengths and limitations of the SHM method.

\subsubsection{Picturing Damage}

Many of the results in the 2-DOF and 10-DOF examples to be presented are explained using a graphical representation of the probability of variation. This visualization tool facilitates interpretation of the variations in $P_{i}^{v a r}\left(t_{m o n}, k\right)$ and helps to demonstrate certain behaviors. A typical plot is in Figure 3.2. All the relevant information for the problem is displayed. The probability of variation, $P^{v a r}$, in a given monitoring cycle, $t_{m o n}=34$, is plotted as a function of the monitoring cycle window, $k$. For $k=1$, only the modal data set taken

during the current monitoring cycle is used in calculating $P^{v a r}$. As $k$ increases, 
additional measured modal data sets from past monitoring cycles are used to calculate $P^{v a r}$. Recall that since the number of monitoring cycles dictates how much past data is available, the maximum for the monitoring cycle window is the number of monitoring cycles. Thus, in the probability of variation plots, the maximum value on the monitoring cycle window axis can not exceed the number of the monitoring cycle, but will oftern be less.

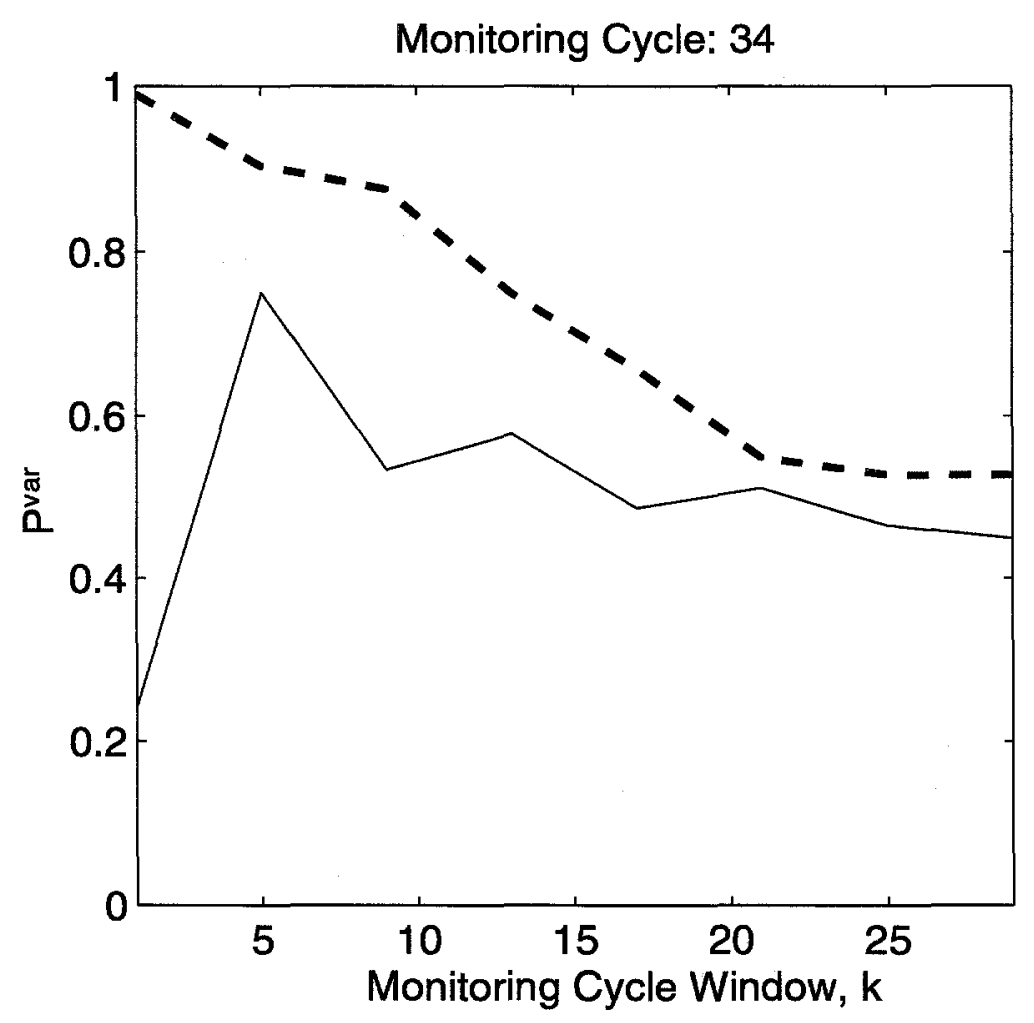

Figure 3.2 Typical probability of variation plot for a given monitoring time

The dashed line represents the alarm function, $P^{\text {alarm }}(k)$, as defined in (2.86), for some choice of $\gamma$. Recall, this alarm function is determined empirically during the initialization phase of the SHM application by calculating the mean and standard deviation of $P^{v a r}$ for each $k$ as $t_{m o n}$ varies. The solid curve is 
the probability of variation curve, and shows $P^{v a r}$ as a function of $k$ for this monitoring cycle. This "curve" is really a set of discrete points joined by lines, but for the purpose of visualization and discussion, it is treated as a curve.

\subsection{Two DOF Shear Structure}

\section{Description of the 2-DOF Model Class}

A 2-DOF shear structure model is considered as both the structure being studied, and the fundamental model for the model class. The model class for this study, $\mathcal{M}_{2}$, is thus "The set of $2-\mathrm{DOF}$ shear structure models with known mass." A typical model is pictured in Figure 3.3. The lumped masses are $m_{1}$

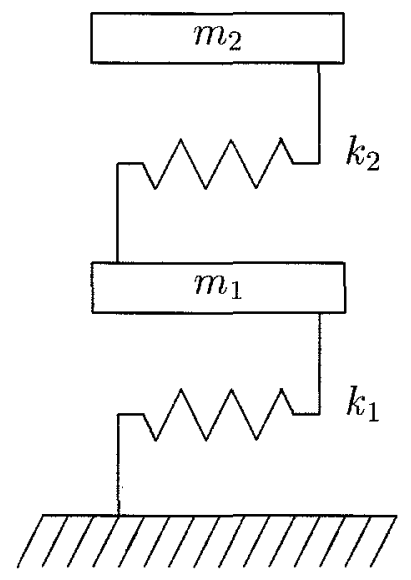

Figure 3.3 This is a representation of a two DOF shear structure model. The spring elements represent resistance to lateral motion between the "floors." The masses are assumed lumped at the "floors."

and $m_{2}$. The elemental stiffness matrices are

$$
\bar{K}_{1}=\left[\begin{array}{cc}
k_{1} & 0 \\
0 & 0
\end{array}\right] \text { and } \bar{K}_{2}=\left[\begin{array}{cc}
k_{2} & -k_{2} \\
-k_{2} & k_{2}
\end{array}\right]
$$


Two possibilities existed for substructuring: one or two substructures. In the first case, SS1, $K_{1}=\bar{K}_{1}+\bar{K}_{2}$ and

$$
K=\theta_{1} K_{1}
$$

In the second case, SS2, $K_{1}=\bar{K}_{1}, K_{2}=\bar{K}_{2}$, and

$$
K=\theta_{1} K_{1}+\theta_{2} K_{2}
$$

Thus, there are either one or two structural parameters depending on the substructuring chosen. This model class is the same as described at the end of Section 2.4.2. The description has been repeated here for convenience.

For all of the tests, the masses, $m_{1}$ and $m_{2}$, are held fixed at the values given in Table 3.2. The "undamaged" value of the stiffnesses, $k_{1}$ and $k_{2}$, are also shown in Table 3.2. The modal parameters for the undamaged model are given in Table 3.3. These values are not obtained by modeling any partic-

\begin{tabular}{|c|c|c|c|c|}
\hline Model Parameter & $m_{1}$ & $m_{2}$ & $k_{1}$ & $k_{2}$ \\
\hline Value & $20 \mathrm{~kg}$ & $10 \mathrm{~kg}$ & $1200 \mathrm{~N} / \mathrm{m}$ & $1000 \mathrm{~N} / \mathrm{m}$ \\
\hline
\end{tabular}

Table 3.2 These are the model parameters for the fundamental model used in this testing. Units are shown only for completeness. They do not represent the values obtained by modeling any particular structure.

ular structure. They are simply chosen to represent a structure with similar interstory stiffnesses, and frequencies in the range of typical civil engineering structures. In order to add a bit of realism, $m_{1}$ is chosen to be greater than $m_{2}$ to reflect that lumping of a structure's mass would put more at an interior point in the model. 


\begin{tabular}{|c|c|c|c|c|}
\hline Modal Parameter & $\omega_{1}$ & $\omega_{2}$ & $x_{1}$ & $x_{2}$ \\
\hline Value & $0.93 \mathrm{~Hz}$ & $2.11 \mathrm{~Hz}$ & {$\left[\begin{array}{c}0.55 \\
0.84\end{array}\right]$} & {$\left[\begin{array}{c}0.60 \\
-0.80\end{array}\right]$} \\
\hline
\end{tabular}

Table 3.3 These are the modal parameters for the fundamental model used in this testing. The modeshape vectors $x_{1}$ and $x_{2}$ have unit norm.

\section{Aims of the 2-DOF Analysis}

The 2-DOF analysis studies the use of the damage measure defined in Chapter 2 to determine the existence, location, and degree of damage in the presence of noisy data. The effect of variations in the number of identified modes, the degree and location of damage, the alarm function, and the substructuring are all considered. The small size of the structure in this case allows for this fairly exhaustive matrix of different possibilities to be explored. For the modal data, the assumption is made that both modeshape components were measured in the one and two mode cases. The problem of multiple most probable models which occurs when only two frequencies are measured is thus avoided. Also, when only one mode is measured, it is the first mode.

\section{Organization of the 2-DOF Study}

The 2-DOF study is organized in a manner which follows the suggested order of application of the SHM method to a real structure. The only difference is that a number of different possible operating conditions are considered at each stage in order to explore the behavior of the method. The first step taken is to determine the number of measured modal data sets required to achieve stability of the undamaged PDF. Then, a study of the variation in $P^{v a r}$ without damage in the structure is conducted. These results demonstrate some of the behaviors predicted in Section 2.5.3 with respect to the values of $P_{i}^{v a r}\left(t_{m o n}, k\right)$ 
as both $t_{m o n}$ and $k$ vary. The effect of the choice of the alarm function, $P_{i}^{a l a r m}(k)$, on the relative frequency of false alarms is analyzed. Finally, the performance of the method with different degrees and locations of damage is considered. Characteristic behavior of $P^{v a r}$ when damage occurred is noted, and some guidelines for using this behavior to separate a true alarm from a false one when an alarm is sounded are established.

\subsubsection{Results of the Testing}

\section{Stabilizing the PDF}

The number of measured modal data sets required to stabilize the most prob-

able model parameters, $\hat{\theta}$, for the undamaged PDF is found as follows. First, a sequence of noisy measured modal data sets is generated. In a real application, this data would represent the data measured in the first part of the initialization phase. From the noisy data, a sequence of most probable models, $\hat{\theta}(n)$, is identified using increasing subsequences of the measured modal data to form the undamaged PDF. These most probable model parameters are plotted against the number of undamaged data sets used to form the undamaged PDF and the trends are noted.

The cases considered are summarized in Table 3.4 and the results are shown. in Figures 3.4 to 3.7. In each figure, results from four different initial sequences are displayed. Although only one set of initial data would be available in a real application, four are generated for this 2-DOF study in order to investigate the consistency of the results. One important observation made from these results is that the SS1 cases converged faster than the SS2 cases for equivalent quantities of modal data. This is not surprising, since in the SS1 cases fewer parameters are identified, so the relative amount of modal data is greater. Having relatively more data pins the parameters values down better. The drawback for the enhanced stability is that SS1 does not give as accurate a 
model of the system as $\mathrm{SS} 2$, so locating damage is not possible.

\begin{tabular}{|c|c|c|}
\hline Test Case & Modal Data & Substructuring \\
\hline \hline 1 & Two Modes & SS2 \\
2 & Two Modes & SS1 \\
3 & First Mode & SS2 \\
4 & First Mode & SS1 \\
\hline
\end{tabular}

Table 3.4 Cases considered in stabilizing the undamaged PDF

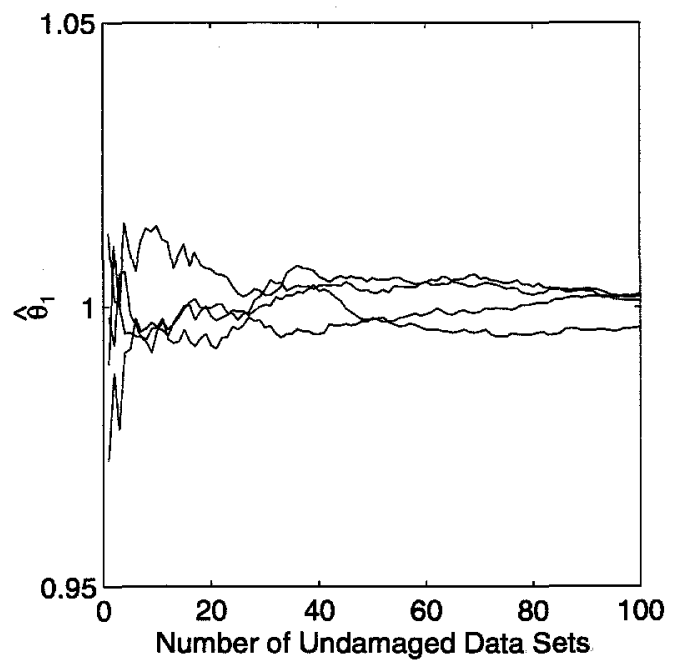

(a) Substructure 1

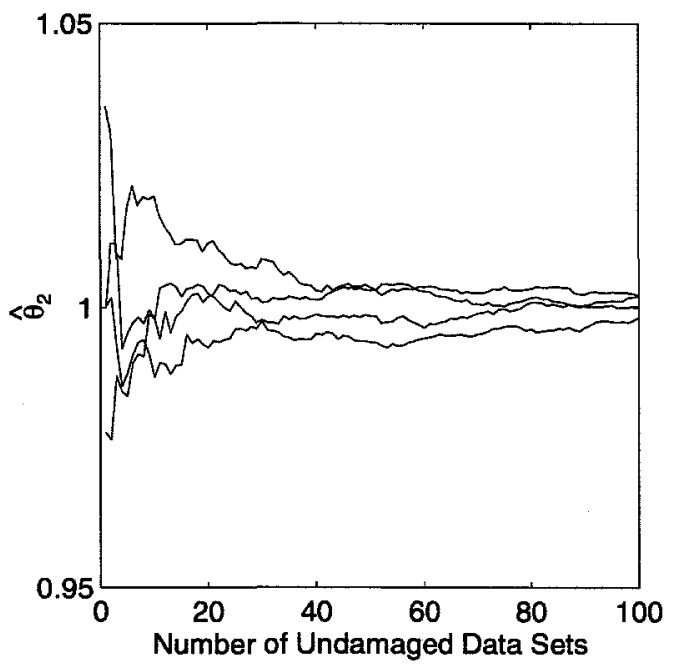

(b) Substructure 2

Figure 3.4 $\hat{\theta}_{1}$ and $\hat{\theta}_{2}$ as functions of the number of modal data sets used to form the undamaged PDF: Test Case 1

For cases 1, 2 and 4, there is a fluctuation of less than $1 \%$ when greater than thirty undamaged data sets are used to form the undamaged PDF. For case 3, significantly many more data sets are required to bring the most probable parameter of the second substructure within the $1 \%$ range. This is an example of an uncertainty problem caused by relative insensitivity of the MOF to 


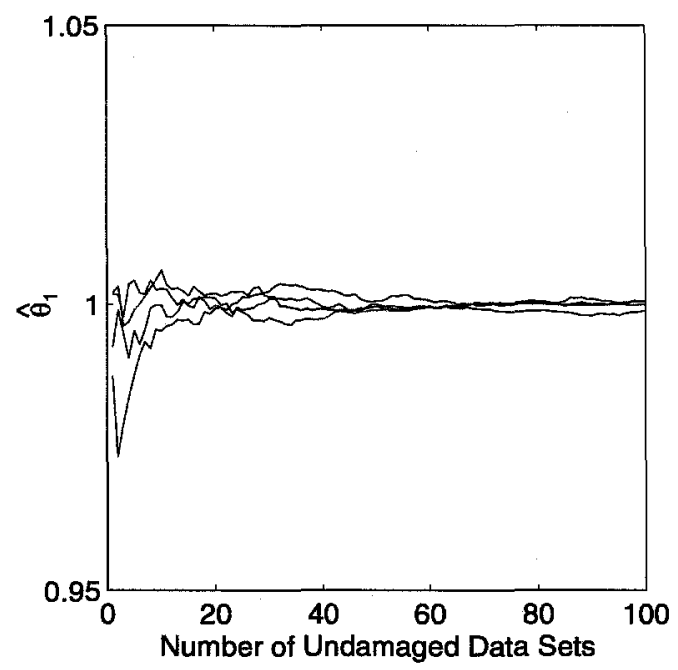

Figure 3.5 $\hat{\theta}_{1}$ as function of the number of modal data sets used to form the undamaged PDF: Test Case 2

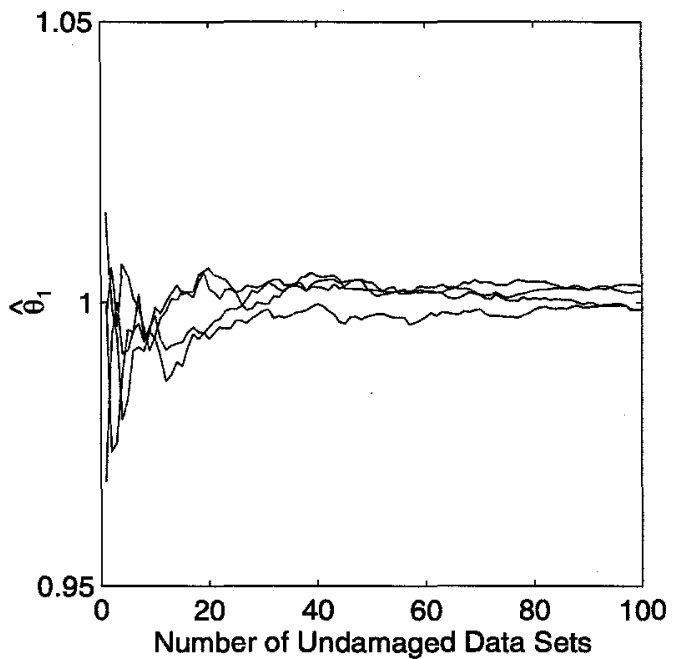

(a) Substructure 1

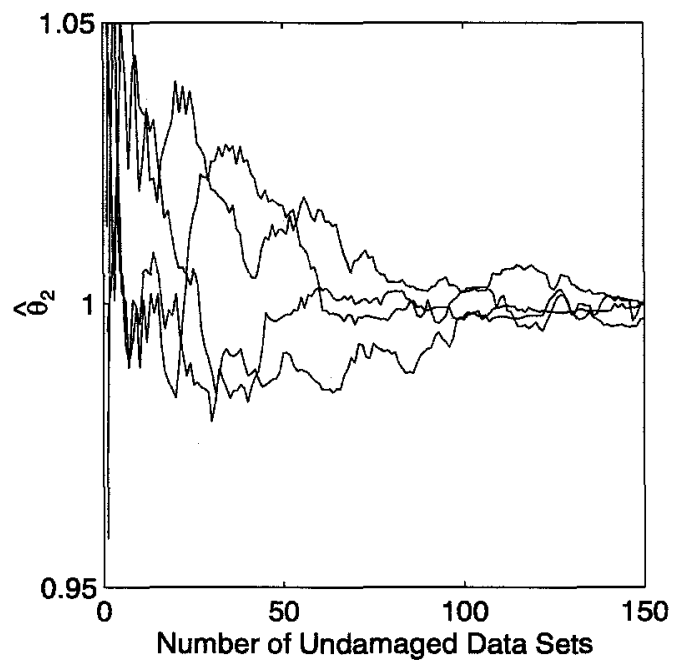

(b) Substructure 2

Figure 3.6 $\hat{\theta}_{1}$ and $\hat{\theta}_{2}$ as functions of the number of modal data sets used to form the undamaged PDF: Test Case 3 


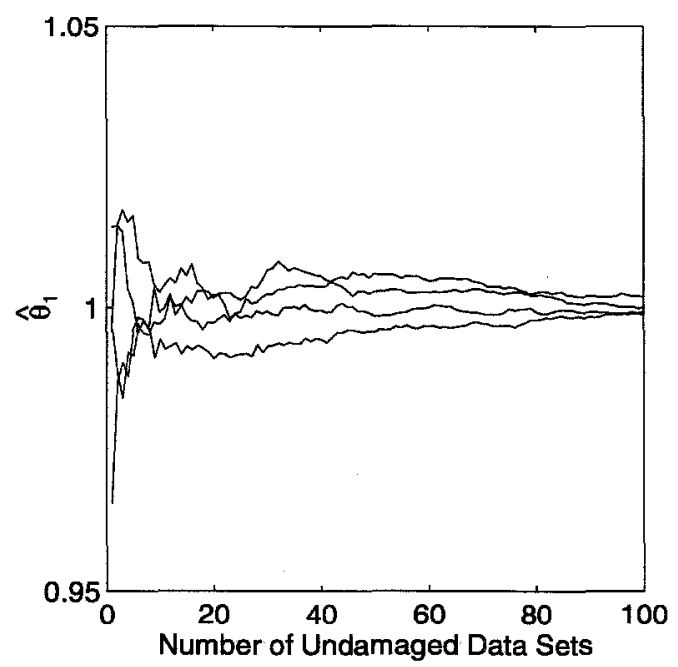

Figure 3.7 $\hat{\theta}_{1}$ as a function of the number of modal data sets used to form the undamaged PDF: Test Case 4

variations in a model parameter. Figure 3.8(a) shows how the first frequency, normalized by its nominal value given in Table 3.3, does not have as strong a dependence on the second model parameter as it does on the first. The steeper curve is the first frequency as a function of the first substructure parameter. The flatter curve is the first frequency as the second substructure parameter changes. Thus, as observed, more data sets should be expected to be required to pin down the second substructure model parameter than the first. The dependence of the second frequency on the parameters is shown in Figure 3.8(b)

The combination of the insensitivity of the first frequency and the small size of this 2-DOF example make working with case 3 extremely difficult. Consistent results are obtained only by performing many more calculations than are needed for the other cases. The exhaustive simulations performed using the other cases would have been prohibitively time consuming, and indicated little more than the fact that working with insensitive parameters might pose some difficulties. Since this is a fact that had already been concluded, only 


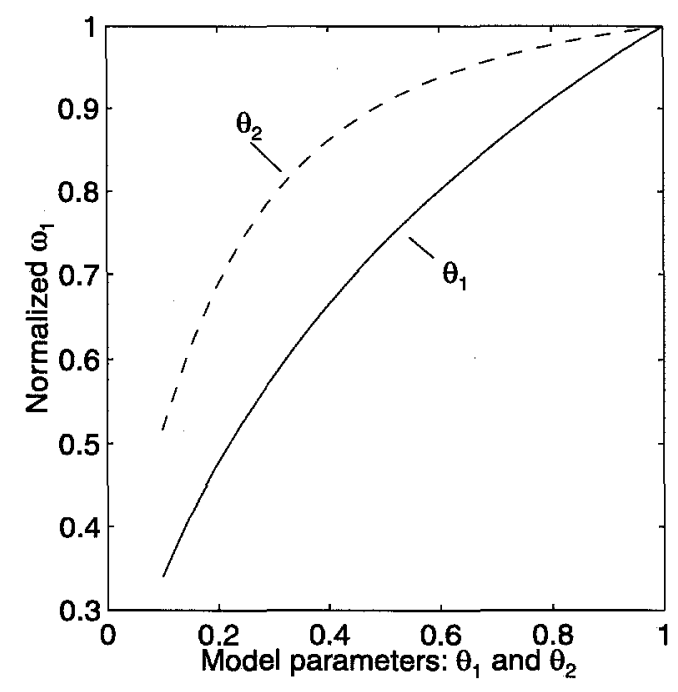

(a) First Frequency

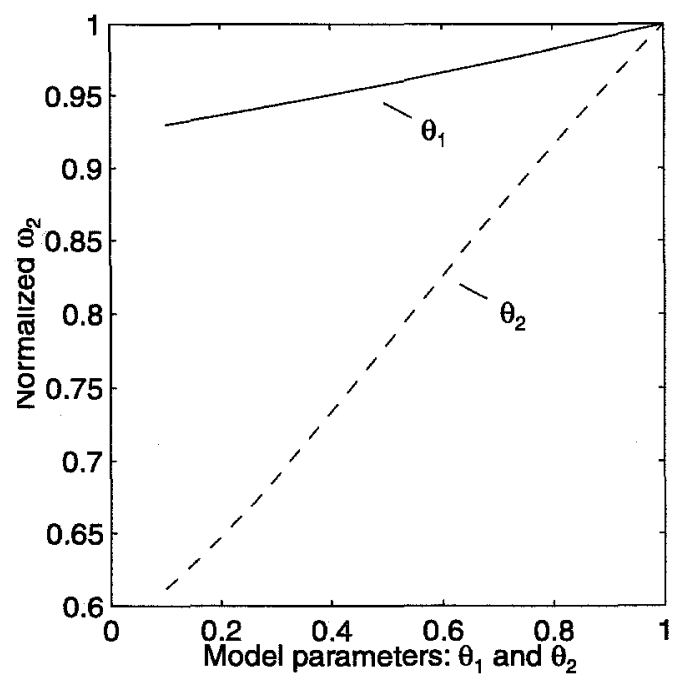

(b) Second Frequency

Figure 3.8 Sensitivities of the modal frequencies with variations in the model parameters

cases 1,2 and 4 are included in the investigation in this work. Based on the previous observations, 32 undamaged data sets are chosen to be used to form the undamaged PDF in these cases.

\section{Undamaged Behavior}

The next step in this analysis is determining the behavior of the $P_{i}^{v a r}\left(t_{m o n}, k\right)$ as $k$ and $t_{m o n}$ varied when no damage is present in the structure. This is accomplished by simulating a large number of monitoring cycles and calculating $P_{i}^{v a r}\left(t_{m o n}, k\right)$ during each monitoring cycle for each substructure. For every $k$, the average and standard deviation of the probability of variation over the simulated monitoring cycles is calculated. These are used to determine the $P_{i}^{a l a r m}(k)$ in the manner described in Section 2.5.4. Rather than choose a single $\gamma$, for this part of the study, $\gamma$ is allowed to vary and the number of alarms for each choice of $\gamma$ is counted. Recall that an alarm is defined to occur in a 
given monitoring cycle any time $P_{i}^{v a r}\left(t_{m o n}, k\right)>P_{i}^{\text {alarm }}(k)$ for any $k$. Taking the ratio between the number of alarms set and the total number of simulated monitoring cycles give a measure of the relative frequency of false alarms.

The actual calculations are performed with the monitoring cycle window varying between $N_{w i n}^{\min }=1$ and $N_{w i n}^{\max }=41$. For all cases, once the undamaged PDF is determined using the appropriate number of undamaged data sets, 200 monitoring cycles are simulated. This number of monitoring cycles is necessary to have the alarm function stabilize because the fluctuations in the probability of variation with $t_{m o n}$ are very large. As will be shown in Section 3.3, the alarming functions can be determined with fewer monitoring cycles when the variations are not as significant. Simulation of the 200 monitoring cycles is performed twice for each case in order to check the consistency of the results. The number of calculations required makes performing a more extensive study with many more repetitions very difficult. Fortunately, the behavior of the alarm functions in each trial of 200 monitoring cycles was very similar. Thus, for each test case, only one of the resulting alarm functions is selected for use in the monitoring phase of the study. Sample alarming functions for $\gamma=1,2$, and 3 for cases 1, 2 and 4 are displayed in Figures 3.9 to 3.11. The plots only show the probability of damage for $P^{v a r}>0.5$ so that the differences in the alarm functions between cases are easier to see. When the value of the alarm function would have exceeded 1 , its value was set to 0.99 so that detecting damage would still be possible.

For small $k, P^{v a r}$ shows significant variation from $50 \%$. This fact is demonstrated by the alarm function exceeding 1 for larger values of $\gamma$. As shown in the next section, damage can still be detected in these cases, but distinguishing false alarms from true alarms becomes difficult. As expected, when the monitoring cycle window is increased (more modal data sets are used to calculate $\left.P^{v a r}\right)$, the degree of variation decreases. Also, the SS1 cases have 


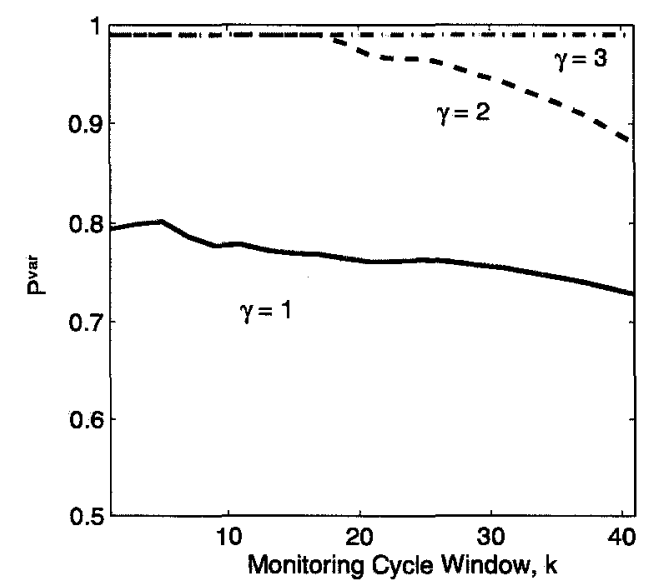

(a) Substructure 1

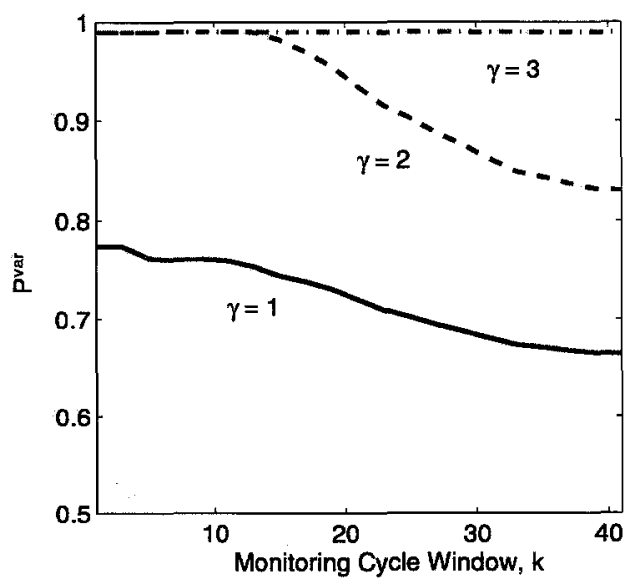

(b) Substructure 2

Figure 3.9 Alarm functions for different values of $\gamma$ : Case 1

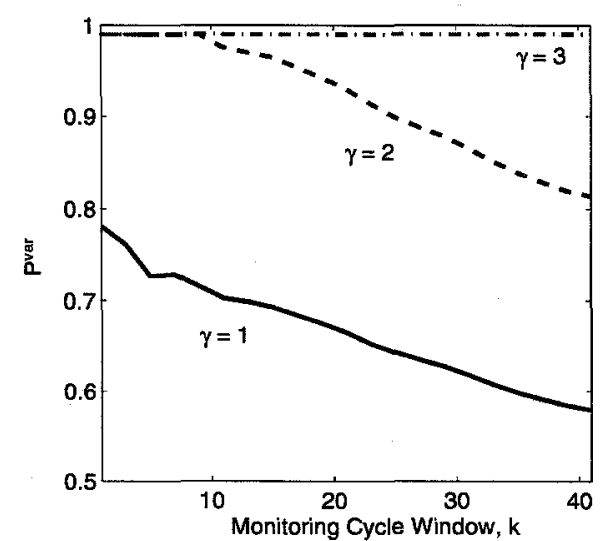

Figure 3.10 Alarm functions for different values of $\gamma$ : Case 2

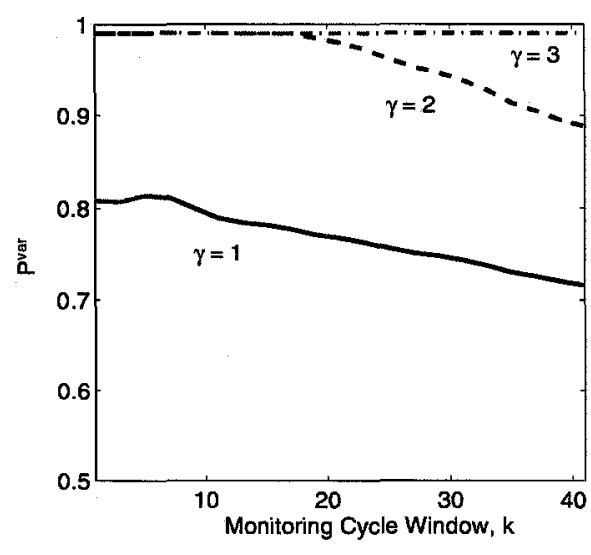

Figure 3.11 Alarm functions for differen values of $\gamma$ : Case 4 
alarm levels which become smaller faster than the alarm levels in the SS2 cases as more data is included. As commented on in the previous section, this result occurs because relatively more data is available to identify the parameters in the SS1 cases.

Figure 3.12 depicts typical probability of variation plots without damage present in the structure. Results are shown for every second cycle over monitoring cycles 51 though 65 . The fixed dashed line is a representative alarm function. The vertical axes are $P^{v a r}$ and the horizontal axes are the monitoring cycle window varying from 1 to 41 . Many of the axis labels have been removed in order to simplify the plots and enable all of them to be contained in one figure. The alarm function was chosen so that a false alarm occurred during the fifty-seventh cycle (i.e. fourth plot).

There are three significant observations made from these plots. First, as predicted in Section 2.5.4, the attenuated effects of past changes are propagated down the sequence of variation probabilities as $t_{m o n}$ increases. Also noted is that no apparent consistent trends in the probability of variation curves are associated with the occurrence of the false alarm. Another way to look at this behavior is that when the alarm level is exceeded at one $k$, there is no correlation with exceeding the level at other values of $k$. In the cases considered, these are generally observed characteristics for false alarms.

Figure 3.12 also revealed one potential problem with the false alarm measure in its current strict definition. Due to the propagation effect, a false alarm at $k=1$ could be propagated down the sequence as a false alarm in future monitoring cycles. Thus, a series of false alarms could be indicated, which are, in fact, the effect of one false alarm. This is an aspect of the method which would have to be taken into account in the guidelines governing the use of the SHM in making decisions. For instance, false alarms set in future monitoring cycles by a spurious peak in the present cycle could be ignored, or 

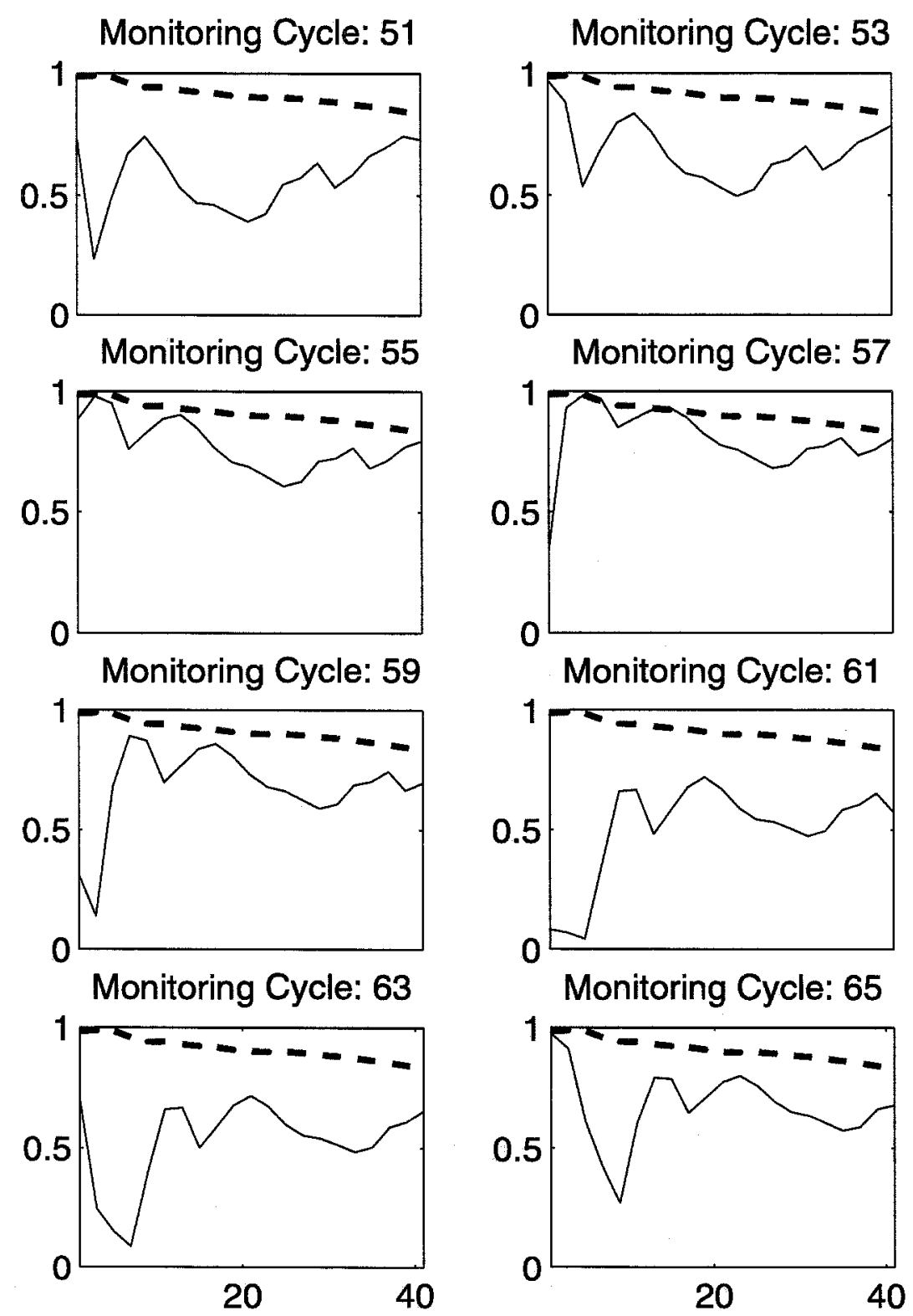

Monitoring Cycle Window Monitoring Cycle Window

Figure 3.12 $P^{\text {var }}$ over sixteen monitoring cycles: No damage. The dashed line is the alarm function for $\gamma=2$. 
the offending data removed from the potentially damaged set.

Two types of relative frequencies of false alarm are generated. The relative frequencies of false alarm shown in Figures 3.13 to 3.15 follow the definition of a false alarm in which exceeding the alarm level for any $k$ is a false alarm. The plots in Figures 3.16 to 3.18 show the relative frequency of false alarms when only excedance at $k=1$ is considered an alarm. For reference, a value of 0.5 would correspond to an alarm in one out of every two monitoring cycles. The probability of false alarms falls off nearly linearly as $\gamma$ increases for all cases considered. This behavior indicates that the variation in $P_{i}^{v a r}$ for each $k$ might be well modeled by a uniform distribution. The root cause of this behavior has not yet been determined, and is part of the ongoing investigation into the use of the SHM method presented in this work. One suggested cause for the behavior is the propagation effect described above. However, the plots for $k=1$ show that the behavior is present even when only the false alarms for a single monitoring cycle window are considered.

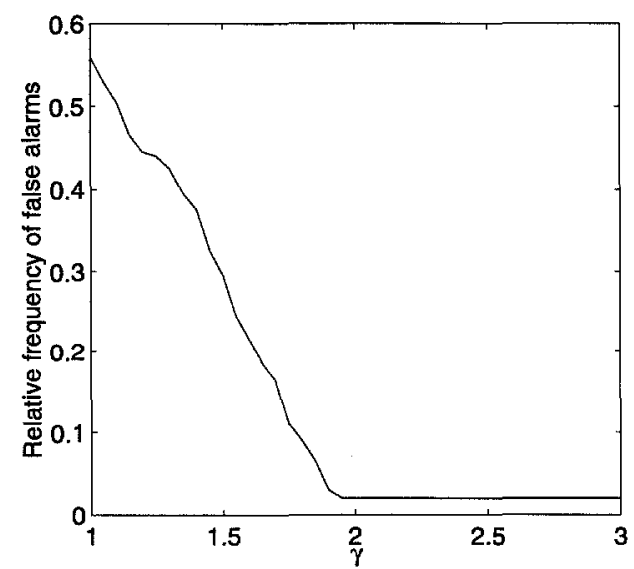

(a) Substructure 1

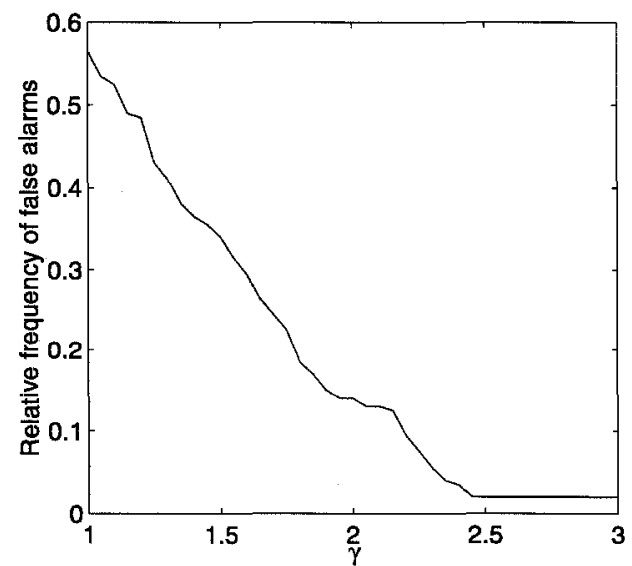

(b) Substructure 2

Figure 3.13 Relative frequencies of false alarm: Case 1, All $k$ 


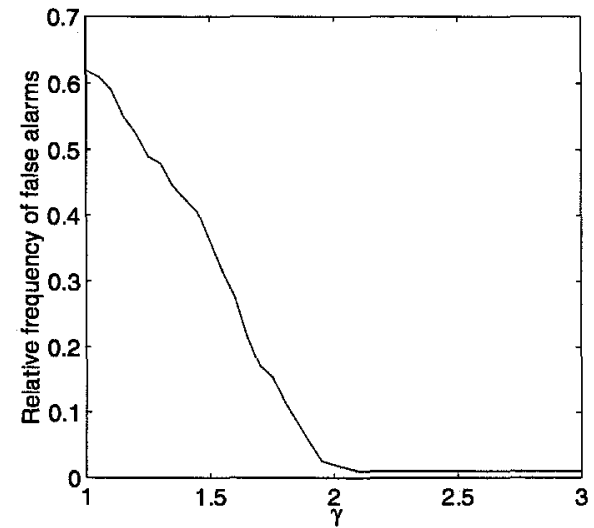

Figure 3.14 Relative frequencies of false alarm: Case 2, All $k$

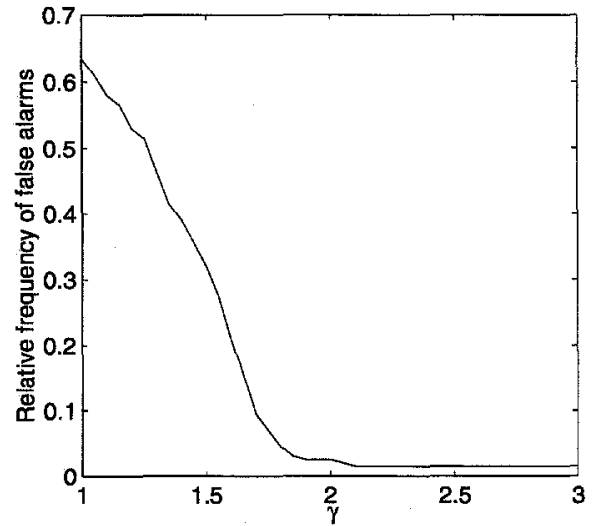

Figure 3.15 Relative frequencies of false alarm: Case 4 , All $k$

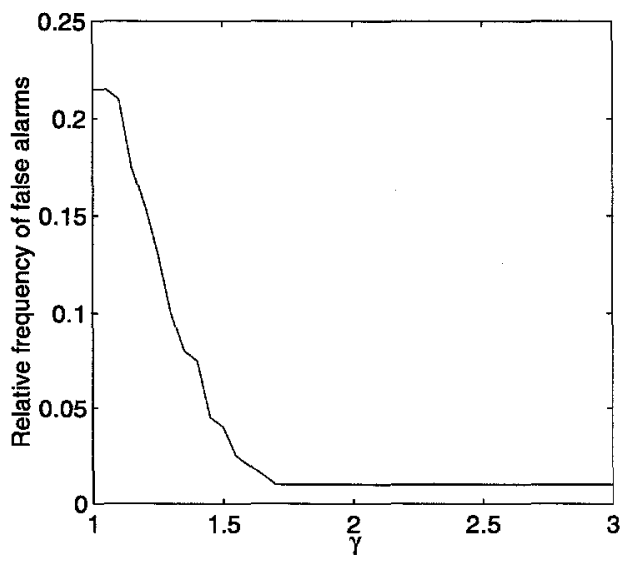

(a) Substructure 1

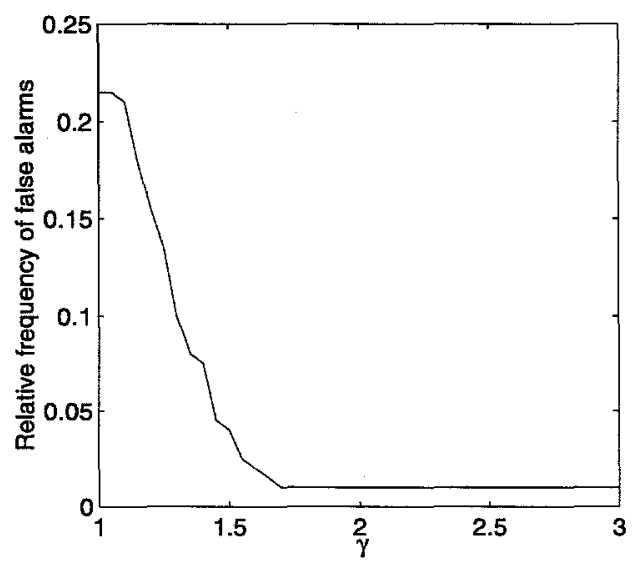

(b) Substructure 2

Figure 3.16 Relative frequencies of false alarm: Case $1, k=1$ 


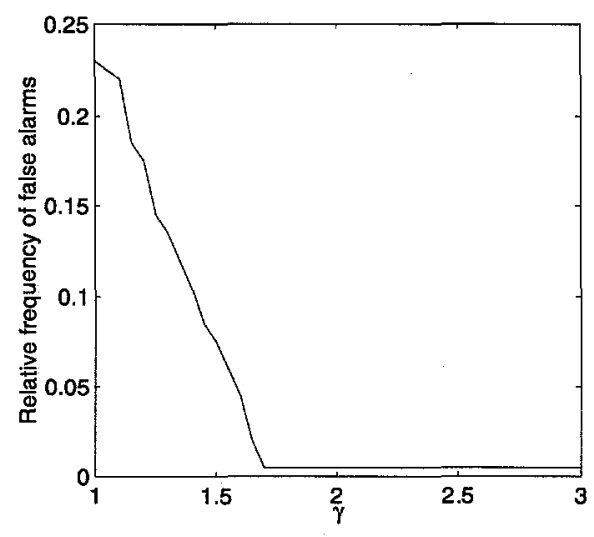

Figure 3.17 Relative frequencies of false alarm: Case $2, k=1$

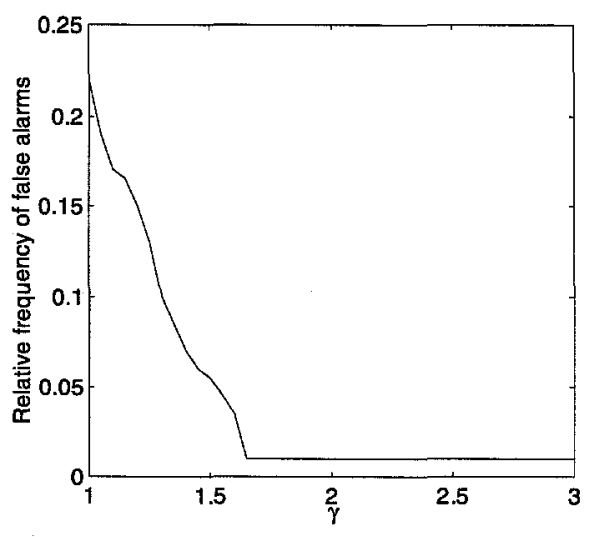

Figure 3.18 Relative frequencies of false alarm: Case $4, k=1$

The relative frequency of alarm plots show that for the SS2 case, selecting $\gamma=2$ for the first parameter, and $\gamma=2.5$ for the second substructure parameter effectively eliminates false alarms. In the SS1 cases, $\gamma=2$ suffices to cut down the number of false alarms. These are the values of $\gamma$ used for the alarm function when the behavior of the SHM method with damage in the structure is analyzed.

\section{Damaged Behavior}

The final step in the analysis is determining how the probability of variation changed when there is damage in the structure. Specifically of interest is how many monitoring cycles are necessary to detect a given damage condition. For this study, damage is considered in each story individually and in both stories together. The degree of damage is also varied.

The results presented in this section are for the cases in Table 3.5. The simulated damage in this table represents the percentage reduction from the nominal values given in Table 3.2. Values for the simulated damage are chosen to exemplify different possible realistic damage scenarios. The case D1 
represents a level damage less than the expected variations due to noise of the most probable model parameters identified from a single data set. Identifying this damage should be difficult when only a few monitoring cycles have passed. However, detecting it should be possible by considering large $k$ after many monitoring cycles have passed. For D2, the simulated damage is at the limits of the variations due to noise for the most probable model parameters identified from a single data set. Case D3 has damage significantly more than the noise threshold. Case, D4, shows the behavior when damage was in multiple locations. Cases in which only the second story is damaged provided no additional insight into the problem beyond the insight gained from the first story damage cases, so they are not discussed.

\begin{tabular}{|c|c|c|c|}
\hline \multirow{2}{*}{$\begin{array}{c}\text { Damage } \\
\text { Case }\end{array}$} & Damage & \multicolumn{2}{|c|}{ Simulated Damage } \\
\cline { 3 - 4 } & Location & $\Delta k_{1}$ & $\Delta k_{2}$ \\
\hline \hline D1 & First Story & $2 \%$ & $0 \%$ \\
D2 & First Story & $5 \%$ & $0 \%$ \\
D3 & First Story & $10 \%$ & $0 \%$ \\
D4 & Both Stories & $10 \%$ & $5 \%$ \\
\hline
\end{tabular}

Table 3.5 Damage cases considered for the 2-DOF example

The testing is conducted with the SHM monitoring system in the monitoring phase. The maximum selection parameter, is $N_{\text {sel }}^{\max }=41$. The effect of damage is introduced in monitoring cycle 42 by using the modal parameters from the shear model with the damage included to create synthetic data. For each combination of test case and damage scenario, a sufficient number of monitoring cycles are then simulated to establish the behavior of the probability of variation curves. For damage cases D2 and D3, no more than ten monitoring cycles are needed. For D1, since the degree of damage was low, as many as thirty monitoring cycles are required. Each set of monitoring simulations is 
repeated five times in order to determine the consistency of the results. Each repeated trial is called a run.

The number of monitoring cycles after the damaging event when the alarm function is first exceeded (first alarm) and the number of additional cycles needed to distinguish the alarm from a false one (verify alarm) are shown in Tables 3.6 to 3.8 . While the first alarm criterion, exceeding the alarm function, is objective, the criteria for verifying an alarm are based on subjectively observing how many points exceed the alarm level and how the probability of damage curve behaves over successive monitoring cycles. Also shown in the Tables 3.6 to 3.8 are the degree of damage measures for the parameter of the substructures. Recall that by the definition for the degree of damage given in (2.88), a reduction in $\theta_{i}$ corresponds to a positive value of the degree of damage. These are calculated using only the damaged data. The damaged data consists of the last $k_{d}$ data sets, where $k_{d}$ is the smallest selection parameter value of the selection parameters at which the first alarm was triggered.

Notice that in case 1 , the degree of damage nearly matches the simulated damage for D1 and D2, but D3 shows significant variation. This happens because the damage is detected in only one cycle in D3. Thus, a single damaged data set is being used to calculate the most probable damaged model, so the values of the identified model are more sensitive to noise than they would be if more data sets had been used. Also, the degree of damage identified in case 2 tends to be less than the elemental degree of damage. In essence, the damage had been "averaged" across the two elements which make up the substructure.

The results from case 4 might seem a bit confusing at first glance since the degree of damage for a given damage case fluctuates about the true damage level. Based in the comments made for the case 2, the damage measure might be expected to vary about a half of the true damage level. The explanation for this behavior is that the substructure stiffness parameter has greater flexibility 


\begin{tabular}{|c|c|c|c|c|c|c|}
\hline \multirow{2}{*}{$\begin{array}{l}\text { Test } \\
\text { Case }\end{array}$} & \multirow{2}{*}{$\begin{array}{l}\text { Simulated } \\
\text { Damage }\end{array}$} & \multirow[b]{2}{*}{ Run } & \multirow{2}{*}{$\begin{array}{l}\text { First } \\
\text { Alarm }\end{array}$} & \multirow{2}{*}{$\begin{array}{l}\text { Verify } \\
\text { Alarm }\end{array}$} & \multicolumn{2}{|c|}{ Degrees of Damage } \\
\hline & & & & & $\Delta \theta_{1}$ & $\Delta \theta_{2}$ \\
\hline \multirow[t]{20}{*}{1} & \multirow[t]{5}{*}{ D1 } & 1 & 17 & 20 & $2.0 \%$ & $-1.0 \%$ \\
\hline & & 2 & 13 & 15 & $2.0 \%$ & $-1.0 \%$ \\
\hline & & 3 & 23 & 30 & $2.0 \%$ & $0.0 \%$ \\
\hline & & 4 & 13 & 24 & $2.0 \%$ & $-1.0 \%$ \\
\hline & & 5 & 15 & 18 & $3.0 \%$ & $-1.0 \%$ \\
\hline & \multirow[t]{5}{*}{ D2 } & 1 & 1 & 6 & $5.0 \%$ & $-2.0 \%$ \\
\hline & & 2 & 2 & 6 & $4.0 \%$ & $0.0 \%$ \\
\hline & & 3 & 2 & 5 & $4.0 \%$ & $0.0 \%$ \\
\hline & & 4 & 1 & 5 & $5.0 \%$ & $0.0 \%$ \\
\hline & & 5 & 2 & 4 & $4.0 \%$ & $-3.0 \%$ \\
\hline & \multirow[t]{5}{*}{ D3 } & 1 & 1 & 2 & $11.0 \%$ & $-4.0 \%$ \\
\hline & & 2 & 1 & 2 & $9.0 \%$ & $-3.0 \%$ \\
\hline & & 3 & 1 & 3 & $7.0 \%$ & $-2.0 \%$ \\
\hline & & 4 & 1 & 2 & $12.0 \%$ & $-2.0 \%$ \\
\hline & & 5 & 1 & 2 & $13.0 \%$ & $-2.0 \%$ \\
\hline & \multirow[t]{5}{*}{ D4 } & 1 & 1 & 2 & $12.0 \%$ & $2.0 \%$ \\
\hline & & 2 & 1 & 2 & $10.0 \%$ & $5.0 \%$ \\
\hline & & 3 & 1 & 2 & $15.0 \%$ & $2.0 \%$ \\
\hline & & 4 & 1 & 2 & $9.0 \%$ & $4.0 \%$ \\
\hline & & 5 & 1 & 2 & $8.0 \%$ & $5.0 \%$ \\
\hline
\end{tabular}

Table 3.6 Results of 2-DOF damage analysis: Case 1 


\begin{tabular}{|c|c|c|c|c|c|}
\hline $\begin{array}{c}\text { Test } \\
\text { Case }\end{array}$ & $\begin{array}{c}\text { Simulated } \\
\text { Damage }\end{array}$ & Run & $\begin{array}{c}\text { First } \\
\text { Alarm }\end{array}$ & $\begin{array}{c}\text { Verify } \\
\text { Alarm }\end{array}$ & $\begin{array}{c}\text { Degree of Damage } \\
\theta_{1}\end{array}$ \\
\hline \hline 2 & D1 & 1 & 10 & 25 & $1.0 \%$ \\
& & 2 & 6 & 10 & $2.0 \%$ \\
& & 3 & 5 & 8 & $2.0 \%$ \\
& & 4 & 9 & 9 & $1.0 \%$ \\
& & 5 & 13 & 13 & $1.0 \%$ \\
\cline { 2 - 6 } & D2 & 1 & 7 & 9 & $2.0 \%$ \\
& & 2 & 3 & 6 & $4.0 \%$ \\
& & 3 & 2 & 5 & $1.0 \%$ \\
& & 4 & 1 & 7 & $4.0 \%$ \\
& & 5 & 2 & 10 & $1.0 \%$ \\
\cline { 2 - 6 } & D3 & 1 & 1 & 3 & $5.0 \%$ \\
& & 2 & 1 & 4 & $6.0 \%$ \\
& & 3 & 2 & 4 & $2.0 \%$ \\
& & 4 & 2 & 4 & $2.0 \%$ \\
& & 5 & 1 & 3 & $6.0 \%$ \\
\cline { 2 - 6 } & & 1 & 1 & 2 & $9.0 \%$ \\
& & 2 & 1 & 3 & $7.0 \%$ \\
& & 3 & 1 & 3 & $7.0 \%$ \\
& & 4 & 1 & 2 & $10.0 \%$ \\
& & 5 & 1 & 3 & $5.0 \%$ \\
\hline
\end{tabular}

Table 3.7 Results of 2-DOF damage analysis: Case 2 


\begin{tabular}{|c|c|c|c|c|c|}
\hline $\begin{array}{l}\text { Test } \\
\text { Case }\end{array}$ & $\begin{array}{l}\text { Simulated } \\
\text { Damage }\end{array}$ & Run & $\begin{array}{l}\text { First } \\
\text { Alarm }\end{array}$ & $\begin{array}{l}\text { Verify } \\
\text { Alarm }\end{array}$ & $\begin{array}{l}\text { Degrees of Damage } \\
\qquad \Delta \theta_{1}\end{array}$ \\
\hline \multirow[t]{20}{*}{4} & \multirow[t]{5}{*}{$\overline{\overline{\mathrm{D} 1}}$} & 1 & $\overline{4}$ & $\overline{16}$ & $2.0 \%$ \\
\hline & & 2 & 6 & 8 & $2.0 \%$ \\
\hline & & 3 & 10 & 30 & $1.0 \%$ \\
\hline & & 4 & 11 & 23 & $2.0 \%$ \\
\hline & & 5 & 4 & 9 & $2.0 \%$ \\
\hline & \multirow[t]{5}{*}{ D2 } & 3 & 3 & 5 & $3.0 \%$ \\
\hline & & 2 & 1 & 4 & $3.0 \%$ \\
\hline & & 3 & 1 & 6 & $7.0 \%$ \\
\hline & & 4 & 3 & 5 & $2.0 \%$ \\
\hline & & 5 & 1 & 5 & $3.0 \%$ \\
\hline & \multirow[t]{5}{*}{ D3 } & 1 & 1 & 2 & $15.0 \%$ \\
\hline & & 2 & 1 & 2 & $10.0 \%$ \\
\hline & & 3 & 1 & 2 & $9.0 \%$ \\
\hline & & 4 & 1 & 2 & $9.0 \%$ \\
\hline & & 5 & 1 & 2 & $9.0 \%$ \\
\hline & \multirow[t]{5}{*}{$\mathrm{D} 4$} & 1 & 1 & 2 & $9.0 \%$ \\
\hline & & 2 & 1 & 2 & $7.9 \%$ \\
\hline & & 3 & 1 & 2 & $10.5 \%$ \\
\hline & & 4 & 1 & 2 & $8.8 \%$ \\
\hline & & 5 & 1 & 2 & $10.5 \%$ \\
\hline
\end{tabular}

Table 3.8 Results of 2-DOF damage analysis: Case 4 
to scale the first interstory stiffness than in case 2 . This is because the first frequency is relatively insensitive to variations in the stiffness for the second story, and no addition constraints were imposed on the second story stiffness by other measured modes.

A final item which needs to be mentioned in regard to the degree of damage in case 1 are the negative values for $\Delta \theta_{2}$ in simulated damage cases D1, D2, and D3. The negative values reflect that the optimal parameter actually increased relative to the undamaged optimal parameter.

One possible avenue of investigation considered when this study began was to determine the relative frequency of missed alarms as a function of $\gamma$ by simulating many damage runs for each damaged condition and counting how often damage was not detected. The relative frequency of missed alarms could have then been plotted on the same plot as the relative frequency of false alarms, and the trade-off in the choice of $\gamma$ determined. The results presented in Tables 3.6 to 3.8 suggested that such an exercise would only be informative for levels of damage which are so low that immediate detection may not be critical. In other words, the probability of variation is sensitive enough to moderate $(5 \%)$ and large $(10 \%)$ degrees of damage that setting $\gamma$ to be the minimum needed to achieve a reasonably low relative frequency of false alarms is acceptable.

The true alarms are distinguished from false alarm by considering the behavior of the probability of variation plots. Figures 3.19 to 3.21 display the typical probability of variation plots as the structure is monitored when damage has occurred in the $42^{\text {nd }}$ monitoring cycle. The fixed dashed line is a representative alarm function. The vertical axes are $P^{v a r}$ and the horizontal axes are the monitoring cycle window varying from 1 to 41. Many of the axis labels have been removed in order to simplify the plots and enable all of them to be contained in one figure. 

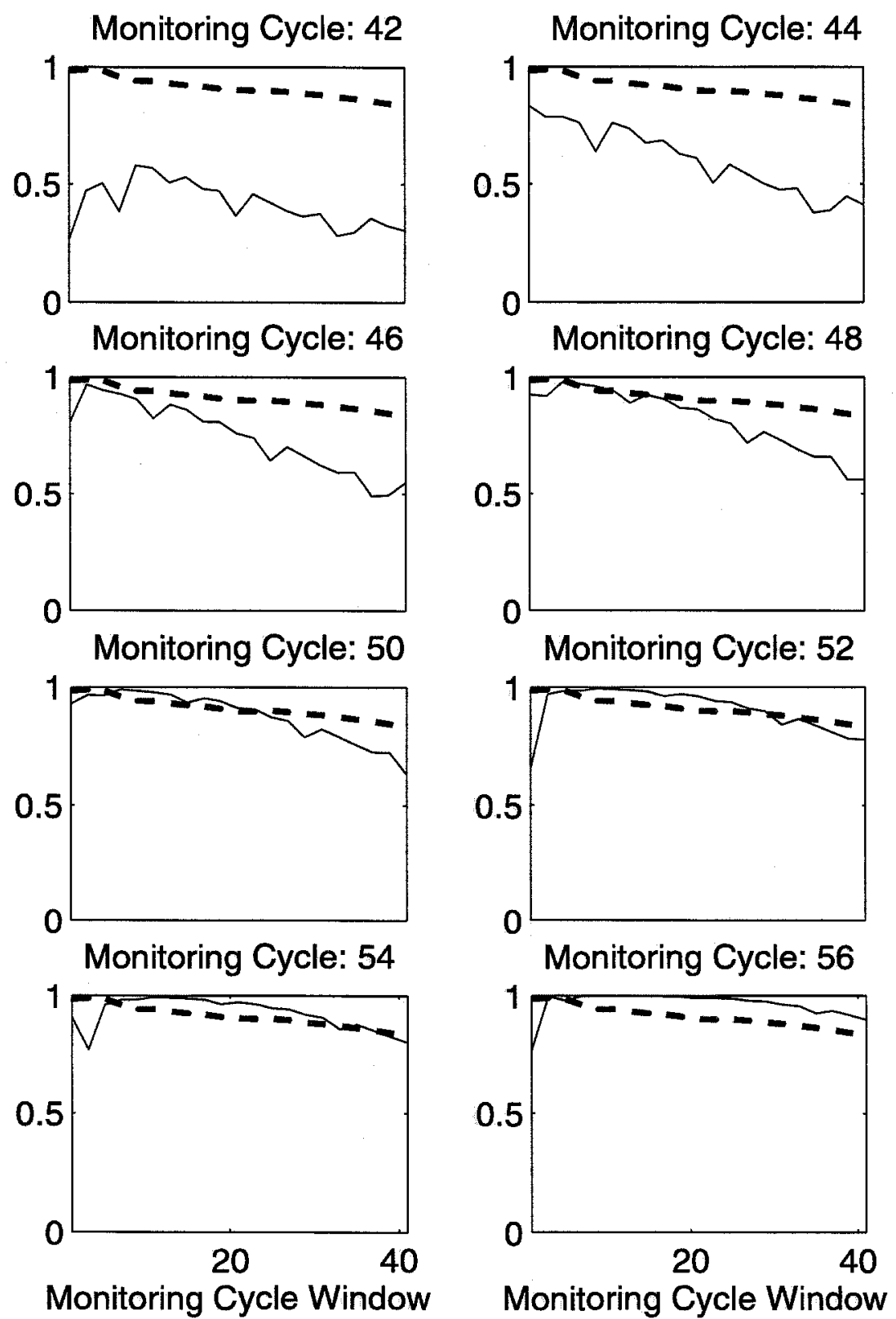

Figure 3.19 $P^{v a r}$ over sixteen monitoring cycles: $2 \%$ damage. The dashed line is the alarm function for $\gamma=2$. 

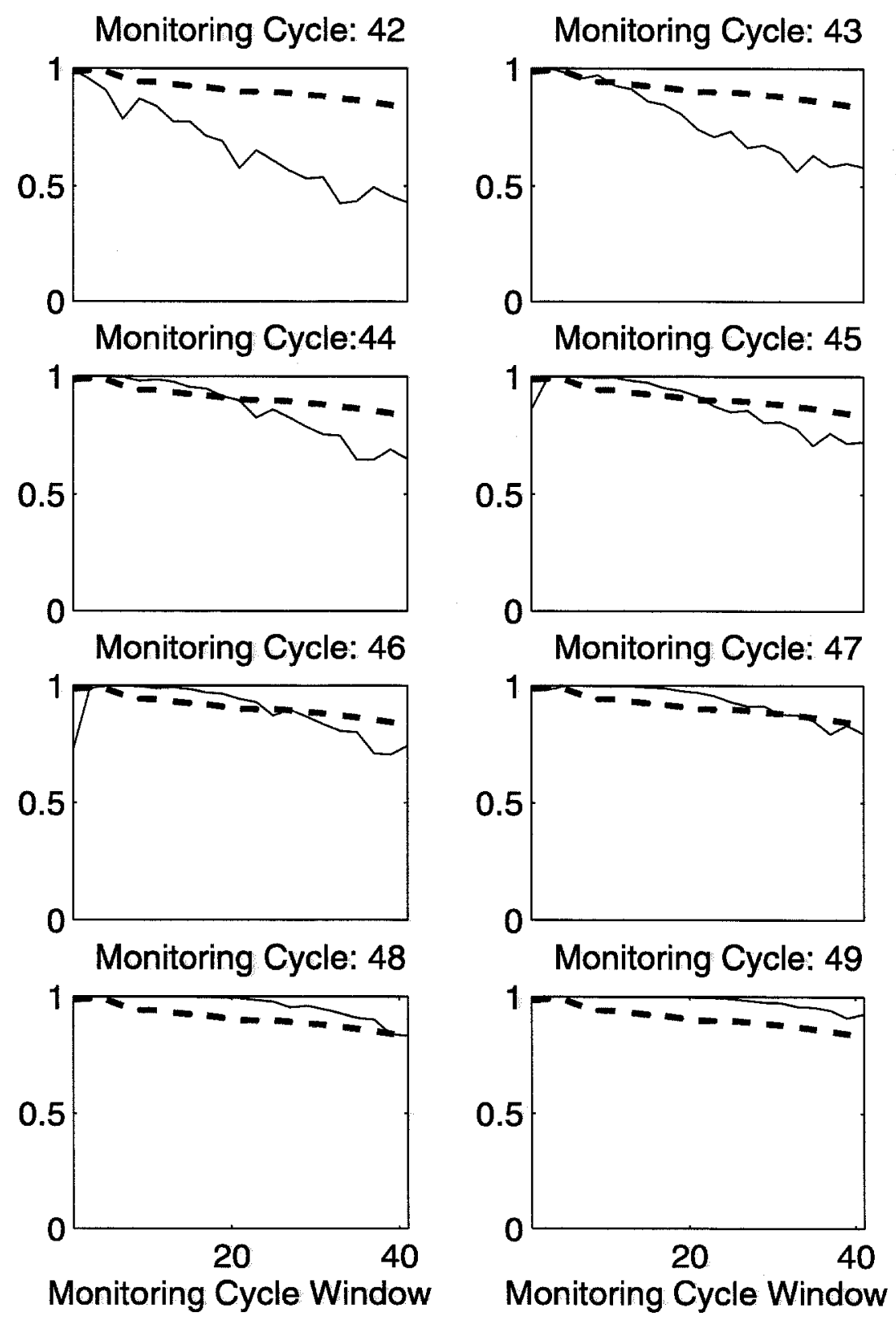

Figure 3.20 $P^{v a r}$ over eight monitoring cycles: $5 \%$ damage. The dashed line is the alarm function for $\gamma=2$. 

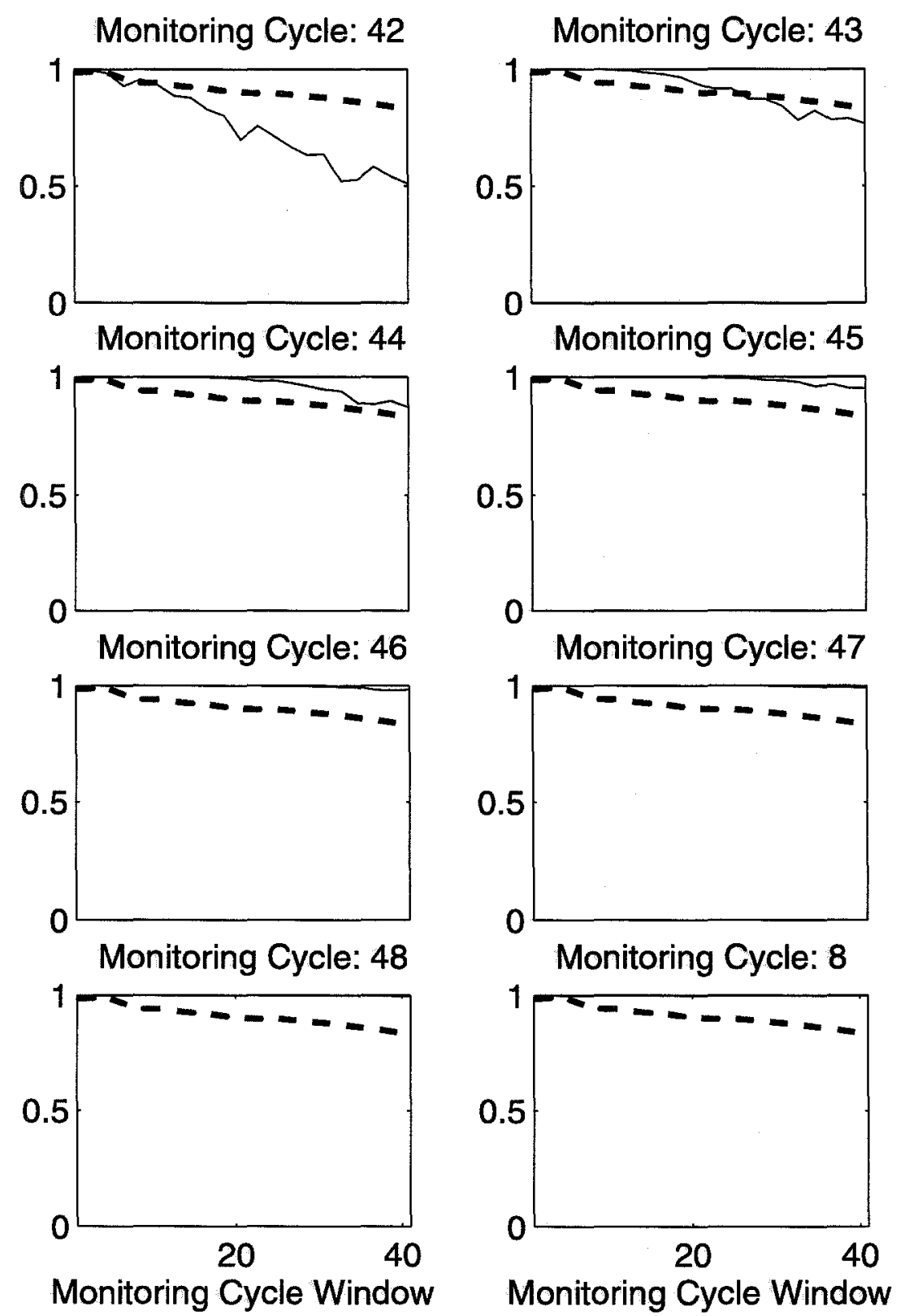

Figure 3.21 $P^{v a r}$ over eight monitoring cycles: $10 \%$ damage. The dashed line is the alarm function for $\gamma=2$. 
Notice in all these cases that the probability of variation curve had an overall increasing trend before and after the alarm function was exceeded. In all of the damaged plots studied, this behavior is observed. The trend varies depending upon the degree of damage. If the damage is sufficiently strong, the probability of variation will be driven to 1 for all $k$ in the monitoring cycle in which the damage was first measured. Essentially, the single damaged data set overcomes the effects of the undamaged data sets as $k$ increases and past undamaged data is included in the evaluation of $P_{i}^{\text {var }}$.

On the other end of the spectrum, small levels of damage may never cause the probability variation to exceed the alarm function when $k$ is small. However, after many monitoring cycles, a great many damaged data sets will be available. Thus, for large $k$, where the effects of noise are reduced, the damage may be detectable. In this case, the plots would be marked by the probability of variation curves exceeding the alarm function for large values of $k$, but remaining within the alarm level for small values of $k$.

As a consequence of the overall increasing behavior, the alarms for the damage events in all cases were triggered by the probability of variation exceeding the alarm function at many values of the monitoring cycle window. Since false alarms tend not to show this correlation, the behavior can be used as an indication of whether a true or false alarm had occurred. An important note is that in only one of the SS2 cases studied did the second substructure stiffness ever exceed the alarm level. Using the observation that the movement of the probability of variation curve before and after the alarm showed no consistent trends, this alarm was correctly categorized as false.

\subsubsection{Observations on the 2-DOF Example}

The results from this study showed many behaviors of the SHM method which carry over into more complex cases. When no damage was present, empirically 
determined alarm functions which effectively eliminated the occurrence of false alarms were found. One item of concern is that a moderately large number of data samples were needed to create stable alarm functions. Taking fewer samples and choosing $\gamma$ conservatively high is one way to handle this problem. Another way would be to reduce the level of fluctuations in the probability of damage for small values of the selection parameter. The variations in the sample statistics for the probability of variation would then settle down to an acceptable level with a smaller number of undamaged data sets. Investigating a reduction in the fluctuations of the probability of variation curves is therefore an avenue of future work.

This study demonstrates that the probability of variation measure defined in Chapter 2 can be used to determine if damage has occurred in the structure. Also, the method is shown to be flexible enough to detect a wide range of degrees of damage. As a first line of evaluation, comparison of the probability of variation with an alarm function can be used to detect whether or not a damaging event may have occurred. In the event that the probability of variation is greater than the alarm level, false and true alarms can be distinguished by considering the manner in which the level was exceeded and, if necessary, the behavior of the probability of variation curves in future monitoring cycles.

The manner in which the alarm function is exceeded provides some way to distinguish types of damage. For large levels of damage, the probability of variation will quickly be driven to 1 for all $k$. For moderate levels of damage, the probability of variation will not shift to 1 immediately, as in the large damage case, but will still tend toward 1 for most of the $k$. Low levels of damage will not cause the probability of variation to exceed the alarm level for small values of $k$ with few monitoring cycles. However, as more damaged data is acquired, the probability of variation should begin to rise above the alarm level for large values of $k$. Alarms when there is no damage should not 
show these behaviors. Thus, recognition of these features can be programmed in to an expert system to assist in the verification of an alarm when one is set.

The 2-DOF example was useful in exhaustively investigating a number of different scenarios which could face the SHM method. Behaviors were identified which appeared for this small problem to be characteristic of the method. Use of the suggested guidelines and determination of whether the noted trends carry over into higher dimensional problems are considered in the following sample application of the SHM method to a 10-DOF shear structure model.

\subsection{Ten DOF Shear Structure}

\section{Description of the 10-DOF Model Class}

In this testing, a $10-\mathrm{DOF}$ shear structure model analogous to the 2-DOF model studied in Section 3.2 is used as the structure. The model class in this case, $\mathcal{M}_{10}$, is thus "The set of $10-\mathrm{DOF}$ shear structure models with known mass." A typical model has the same form as the 2-DOF model depicted in Figure 3.3, except there are ten lumped masses and interstory stiffnesses, numbered from the bottom to the top. Thus, $m_{i}$ and $k_{i}$ are the mass and stiffness of the $\mathrm{i}^{\text {th }}$ story. For all of the tests, the masses, $m_{1}, \ldots, m_{10}$, were held fixed at the values given in Table 3.9. The "undamaged" value of the stiffnesses, $k_{1}, \ldots, k_{10}$ are also shown in Table 3.9. As in the 2-DOF example, these mass and stiffness values were not obtained by modeling a particular structure.

The fundamental model had ten elemental stiffness matrices of a similar form to the 2-DOF stiffness matrices, except that $\bar{K}_{i} \in \mathbb{R}^{10 \times 10}$. Many possibilities existed for substructuring. The two which were considered in this study are shown in Table 3.10. The same notation is used as in the 2-DOF example. 


\begin{tabular}{|c|c||c|c|}
\hline Mass & Value $(\mathrm{kg})$ & Stiffness & Value $(\mathrm{N} / \mathrm{m})$ \\
\hline$m_{1}$ & 30 & $k_{1}$ & 48000 \\
$m_{2}$ & 30 & $k_{2}$ & 48000 \\
$m_{3}$ & 30 & $k_{3}$ & 48000 \\
$m_{4}$ & 20 & $k_{4}$ & 44000 \\
$m_{5}$ & 20 & $k_{5}$ & 44000 \\
$m_{6}$ & 20 & $k_{6}$ & 44000 \\
$m_{7}$ & 20 & $k_{7}$ & 44000 \\
$m_{8}$ & 20 & $k_{8}$ & 44000 \\
$m_{9}$ & 20 & $k_{9}$ & 40000 \\
$m_{10}$ & 10 & $k_{10}$ & 40000 \\
\hline
\end{tabular}

Table 3.9 These are the model parameters for the fundamental model used in the 10-DOF testing. Units are shown only for completeness. They do not represent the values obtained by modeling any particular structure.

\begin{tabular}{|c|c|c|}
\hline Substructuring & Description & \# Parameters \\
\hline \hline SS1 & Every element is a substructure & 10 \\
SS2 & Every two stories is a substructure & 5 \\
\hline
\end{tabular}

Table 3.10 Substructuring possibilities considered for the 10DOF example 


\section{Measured Data and Damage Scenarios}

In this analysis, two different possibilities for the measured data were considered. In the first case, M2, only two modes were measured, but the structure was instrumented at all the floors. In the second case, M5, five modes were measured, but the structure was only instrumented at floors $3,6,8,10$. This choice of sensor distribution was made based on looking at the modeshape plots and subjectively judging where the measurements would pick up the greatest variations in the modeshape components. Other distributions were not investigated as this was a study of the SHM method, not optimal sensor location. The combination of substructurings and types of available modal data lead to four test cases. These are summarized in Table 3.11. Note that in both M2 and M5, 20 modal parameters are available.

\begin{tabular}{|c|c|c|}
\hline Test Case & Modal Data & Substructuring \\
\hline \hline 1 & M2 & SS1 \\
2 & M5 & SS1 \\
3 & M2 & SS2 \\
4 & M5 & SS2 \\
\hline
\end{tabular}

Table 3.11 Different test cases considered in 10-DOF example

The three damage scenarios presented are shown in Table 3.12. Cases D1 and D2 gave damage below and above, respectively, the expected variations due to noise of the most probable model parameters identified from a single data set. The third situation, D3, was included in the analysis to see how the SHM method performed with damage in multiple locations. 


\begin{tabular}{|c|c|c|}
\hline $\begin{array}{c}\text { Damage } \\
\text { Case }\end{array}$ & Damage & $\begin{array}{c}\text { Simulated } \\
\text { Damage }\end{array}$ \\
\hline \hline D1 & Fifth Story & $\Delta k_{5}=5 \%$ \\
D2 & Fifth Story & $\Delta k_{5}=15 \%$ \\
D3 & Stories 1, 2, and 3 & $\Delta k_{1}=10 \%, \Delta k_{2}=20 \%$, and $\Delta k_{3}=15 \%$ \\
\hline
\end{tabular}

Table 3.12 Damage cases considered for the 10-DOF example

\subsubsection{Initialization Phase}

For the given levels of noise on the modal parameters, stabilization testing indicated that about 50 or so undamaged runs were necessary for variations in the most probable model parameters of less than $0.5 \%$. For this study, 60 undamaged data sets were used to calculate the undamaged PDF. The reason for this slightly conservative choice is that only one sequence of modal measurements was used to establish the necessary number of undamaged measurements.

The alarm functions were determined from 30 monitoring cycles of undamaged data. Unlike the 2-DOF alarm functions, the 10-DOF alarm functions appear to converge to stable values with fewer monitoring cycles. This was believed to have occurred because the fluctuations in $P^{\text {var }}$ for this case were not as large as they were in the $2-\mathrm{DOF}$ example. As mentioned in Section 3.2.2, smaller fluctuations in the probability of variation imply that the variations in the statistics will settle down to an acceptable level with fewer samples. The fluctuations were themselves smaller because more data, both in an absolute and a relatively sense, was available than in the 2-DOF case. Four sample alarm functions for case 1 are depicted in Figure 3.22. For all of the alarm functions, $\gamma=2.8$ was chosen. This choice reflects a conservative approach based on the fact that a small sample set was used to establish the alarm levels. 

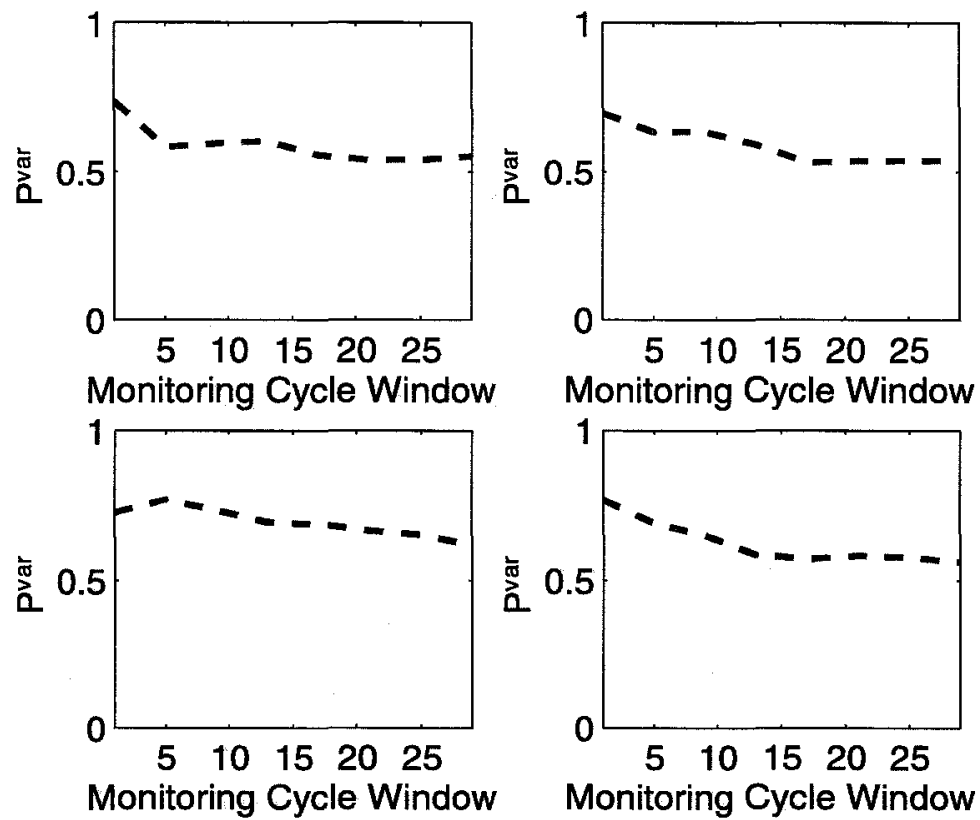

Figure 3.22 Alarm functions for four parameters

\subsubsection{Monitoring Phase}

For each of the testing scenarios, the structure was assumed to have been monitored for $t_{m o n}=29$ cycles, and damaged at $t_{m o n}=30$. Tables 3.13 to 3.16 show the number of monitoring cycles taken to sound the alarm for damage (not possible in all cases), the number of cycles needed to distinguish the alarm from a false one and the degree of damage for every parameter when the alarm was first triggered.

The "*" for the damage case D1 indicates that nothing conclusive could be determined in this case. Theoretically, if sufficient data is taken, the effects of noise could be reduced to a small enough level that any degree of damage would be detectable. The results in testing with the D1 case indicate that there may be practical limitations to the applicability of this concept. First, the number of cycles needed to reduce the noise level may be unrealistically large. Also, in order to make conclusions based on small changes in the prob- 


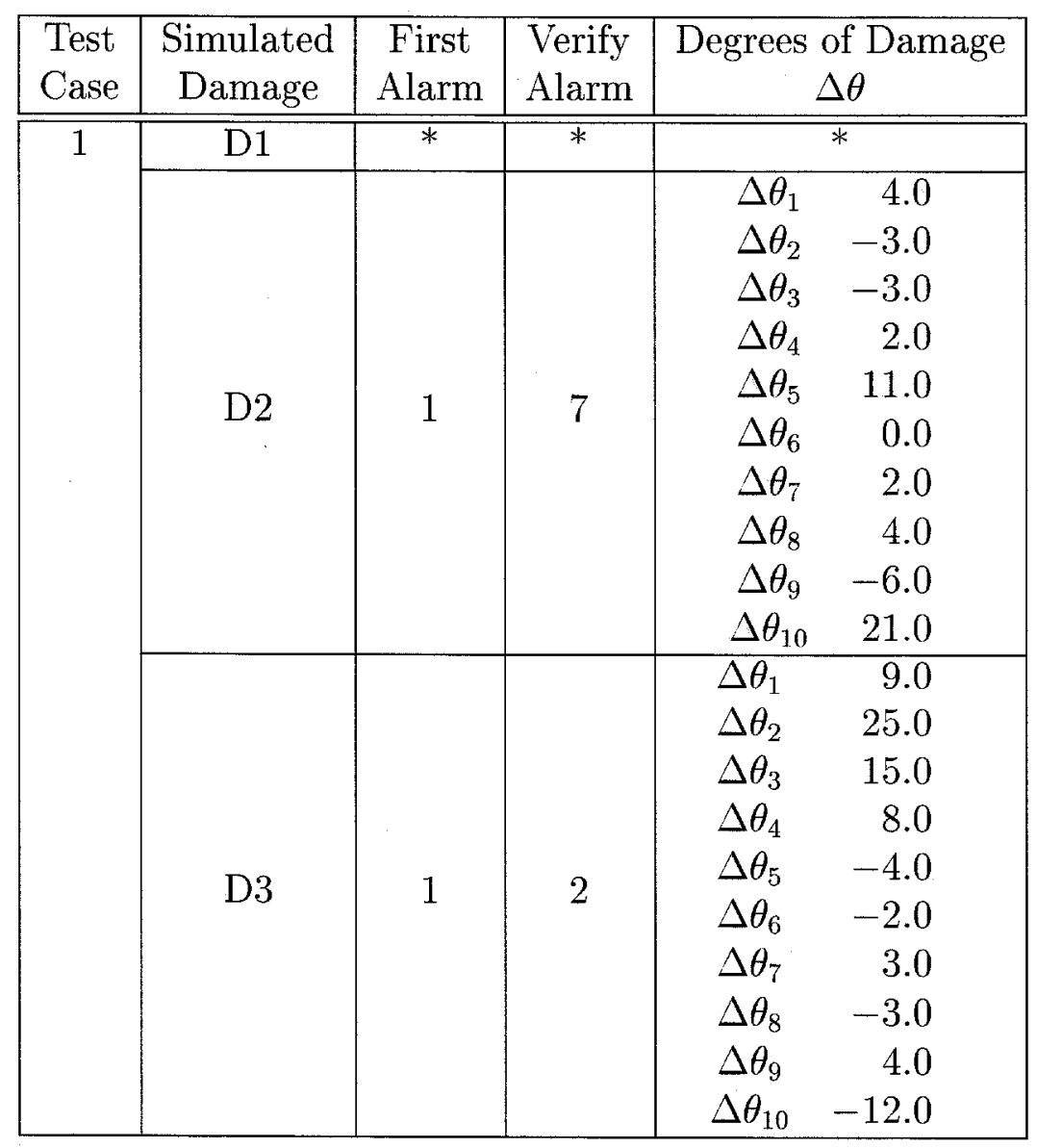

Table 3.13 Results of 10-DOF damage analysis: Case 1 


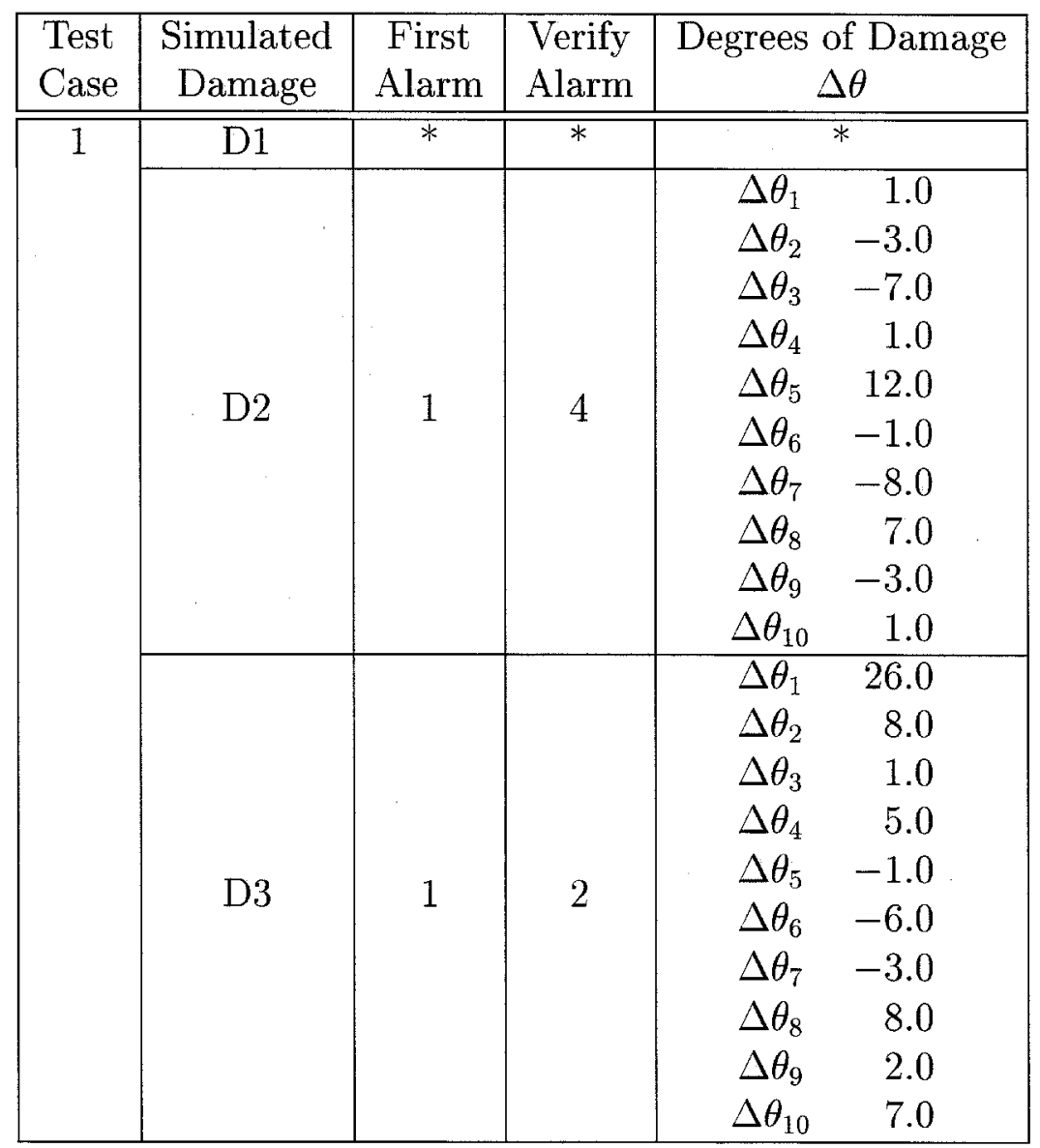

Table 3.14 Results of 10-DOF damage analysis: Case 2 


\begin{tabular}{|c|c|c|c|c|}
\hline $\begin{array}{l}\text { Test } \\
\text { Case }\end{array}$ & $\begin{array}{c}\text { Simulated } \\
\text { Damage }\end{array}$ & $\begin{array}{l}\text { First } \\
\text { Alarm }\end{array}$ & $\begin{array}{l}\text { Verify } \\
\text { Alarm }\end{array}$ & $\begin{array}{c}\text { Degrees of Damage } \\
\Delta \theta\end{array}$ \\
\hline \multirow[t]{3}{*}{1} & $\overline{\mathrm{D} 1}$ & $\bar{*}$ & * & * \\
\hline & D2 & 1 & 5 & $\begin{array}{lr}\Delta \theta_{1} & 5.0 \\
\Delta \theta_{2} & -6.0 \\
\Delta \theta_{3} & 13.0 \\
\Delta \theta_{4} & 2.0 \\
\Delta \theta_{5} & 9.0\end{array}$ \\
\hline & D3 & 1 & 2 & $\begin{array}{lr}\Delta \theta_{1} & 11.0 \\
\Delta \theta_{2} & 16.0 \\
\Delta \theta_{3} & -3.5 \\
\Delta \theta_{4} & 2.0 \\
\Delta \theta_{5} & -1.0\end{array}$ \\
\hline
\end{tabular}

Table 3.15 Results of 10-DOF damage analysis: Case 3

\begin{tabular}{|c|c|c|c|c|}
\hline $\begin{array}{l}\text { Test } \\
\text { Case }\end{array}$ & $\begin{array}{l}\text { Simulated } \\
\text { Damage }\end{array}$ & $\begin{array}{l}\text { First } \\
\text { Alarm }\end{array}$ & $\begin{array}{l}\text { Verify } \\
\text { Alarm }\end{array}$ & $\begin{array}{l}\text { Degrees of Damage } \\
\qquad \Delta \theta\end{array}$ \\
\hline \multirow[t]{3}{*}{1} & D1 & * & $*$ & * \\
\hline & D2 & 1 & 9 & $\begin{array}{lr}\Delta \theta_{1} & 1.0 \\
\Delta \theta_{2} & 0.0 \\
\Delta \theta_{3} & 8.0 \\
\Delta \theta_{4} & 4.0 \\
\Delta \theta_{5} & -2.0\end{array}$ \\
\hline & D3 & 1 & 2 & $\begin{array}{lr}\Delta \theta_{1} & 15.0 \\
\Delta \theta_{2} & 4.0 \\
\Delta \theta_{3} & -2.0 \\
\Delta \theta_{4} & -2.0 \\
\Delta \theta_{5} & 0.0\end{array}$ \\
\hline
\end{tabular}

Table 3.16 Results of 10-DOF damage analysis: Case 4 
ability of variation curves, the alarm levels have to be known with enough confidence that exceeding them by small levels could be treated as significant indicators of damage. Finally, some types of "noise" are not introduced through the data, so taking more data samples may not help reduce their effects. For instance, within the minimization scheme are multiple levels of iterative minimization steps which are stopped when certain criteria are met. Thus, the exact minimum of the MOF is not actually calculated. This can be viewed as a type of noise. This type of noise can have a significant effect on parameters for which the MOF is highly insensitive.

The progressively increasing trend in the $P^{v a r}$ associated with the 2-DOF damaged substructure was also observed in these examples. Figure 3.23 shows an example for the fifth substructure of case 1, with damage D2. In this case, the alarm appeared to be false at first, since after two cycles the alarm level was no longer exceeded. However, by the seventh cycle, the probability of variation curve had grown systematically beyond the alarm level for a sufficient number of monitoring cycles that the substructure was declared damaged.

For test cases 1 and 2, the D2 damage case was positively detected in story without indicating damage in any other members. The substructuring test cases, 3 and 4 , were both able to positively identify damage in the third substructure, which corresponded to stories 5 and 6 . However, test case 4 also gave an alarm for damage in the first story that appeared to be a true alarm.

Damage case D3 was also successfully detected in all four test cases. Only in test cases 2, 3, and 4 was the location determined without error. In test case 1 , the damage in story 3 was not found. Damage was positively identified in story 4 , where there was no damage. 

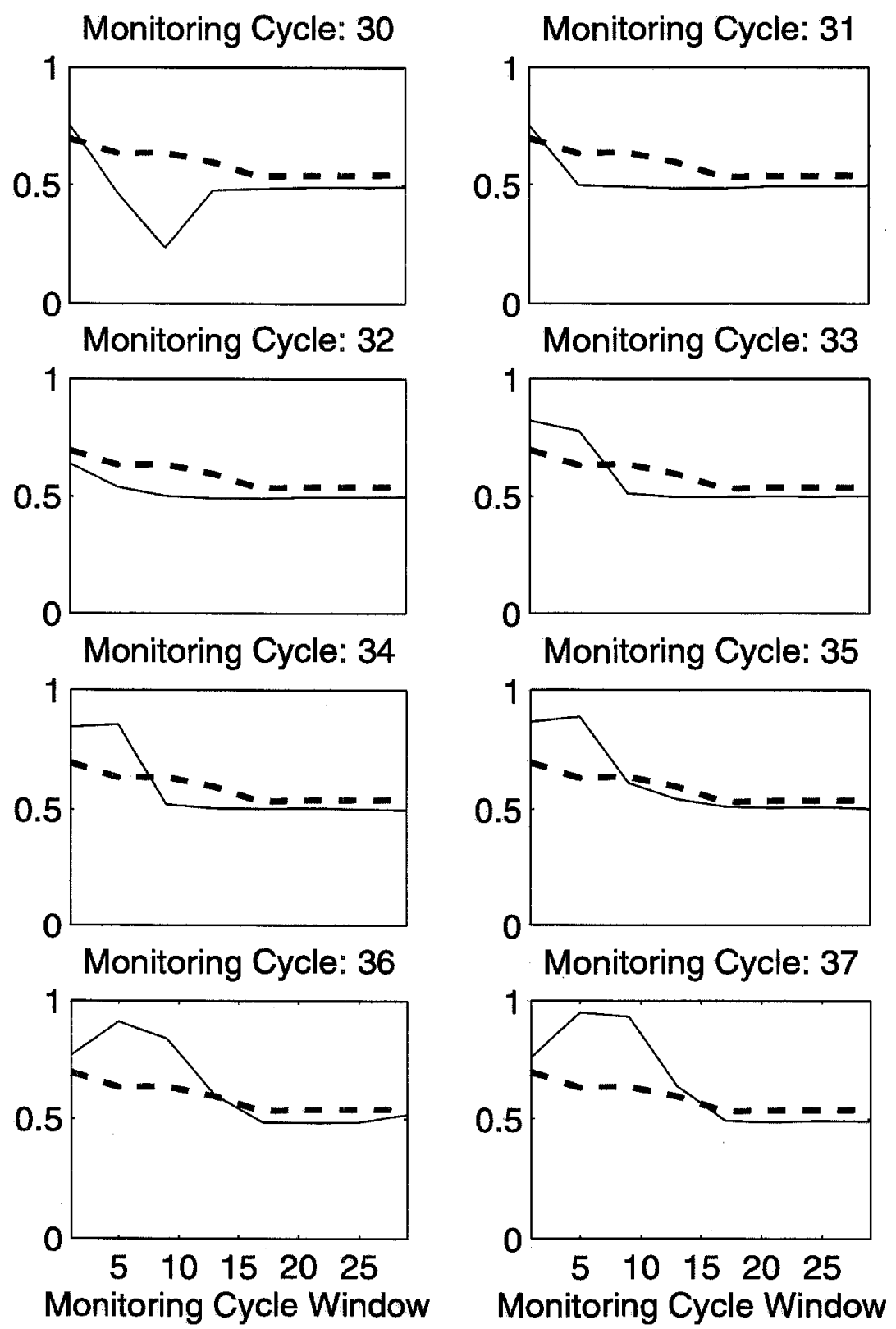

Figure 3.23 $P^{d a m}$ over eight monitoring cycles: Test Case 1, Damage Case D2, Fifth Story Substructure Parameter, $\gamma=2.8$ 


\subsubsection{Concluding Remarks on the 10-DOF Example}

The sample cases presented in this section showed that the behaviors observed in the 2-DOF example carry over to the $10-\mathrm{DOF}$ example. The results also demonstrated that damage could be successfully detected and located. However, as the size of the problem had increased, some difficulties arose which were not a significant problem in the 2-DOF example. For instance, damage was detected in locations where there was none. Also, the minimization scheme used may have limited the capability of the method to determine very low levels of damage. Given that the method appeared to otherwise handle the SHM problem quite well, further work in resolving these limitation issues is warranted. 


\section{Chapter 4}

\section{Conclusions and Future Work}

\section{$4.1 \quad$ Future Work}

Throughout this work, phrases similar to, "This matter is left for future work." have appeared. This is not an indication of a lack of effort in the development of the SHM method which was presented. Rather, it reflects the fact that the method discussed in this work is a transitional step from theory to implementation. This SHM method is therefore by no means the end of an old story. On the contrary, it is the beginning of a new one. Because of this, the application of the technique requires additional testing, and possibilities for improvement are numerous. This section summarizes some of the more important suggested avenues of further investigation.

\subsubsection{Additional Testing}

The primary focus of future efforts should be on continued testing with more realistic simulated structures and experimental structures in order to see how well the method will perform in practice. Cases in which different modal parameters have different noise levels and the number of available modal parameters in different measurements is allowed to vary are examples of matters 
which should be considered. Investigating what types of damage in a structure the SHM can realistically detect is also necessary. Thus, determining how member damage in the true structure is reflected in the degree of damage in the substructure which contains those members should be pursued. This testing should continue the exploration into the characteristics of the probability of variation function in various scenarios so that more detailed guidelines for its use can be developed. In this manner, an interpretive logic for use by an expert system or end user can be created.

Applying the method to time-varying models is another pursuit of interest. The most obvious problems to treat would be those in which gradual changes in the structure due to diurnal or seasonal variations occur. In such applications, a time-varying undamaged PDF based on sets of undamaged data associated with different conditions could be used to account for these changes not due to damage. Efforts in studying application of the SHM method to the problem of systematic change without damage will lead to a more robust monitoring method.

The assumption was made in this paper that the updated PDF had a global maximum. Cases can exist wherein multiple local maxima are present. For example, in the 2-DOF shear model, if only the two frequencies are measured, then there are two local maxima of the PDF. Sufficient modeshape information usually mitigates the multiple maxima problem. There are instances, however, when either only frequency information is available, or the modeshape information is so noisy that the frequency measurements control the MOF. Thus, research into the application of the method when multiple maxima are present will be useful.

The final item of interest in testing does not pertain to this work alone, but speaks of a general need in the SHM community. As the survey in Section 1.2 demonstrated, many different approaches to global SHM exist. However, un- 
like other more mature fields, SHM does not have a body of benchmark examples by which the different methods can be compared. Such examples would be a common reference by which to judge different methods. Also, researchers without direct access to experimental facilities could be provided with opportunities to test their methods using experimental data generated by others. Establishment of these standard problems is therefore an important step in helping to further efforts in the entire SHM field.

\subsubsection{Improving the Method}

\section{A Better Alarm Function}

The alarm function used in this work was found in an empirical fashion. However, knowing that the probability of variation sequence has a random walk behavior implies that a methodology for choosing the alarm function which possesses some analytical basis could be formulated. Having a better method

for choosing the alarm function which reduces the number of data measurements required in the initialization phase of the SHM technique would be a significant improvement. One possible way to handle this further pursuit is by formulating another layer of Bayesian analysis in which the plausibility of the probability of variation sequences conditional on the undamaged and potential damaged PDFs is determined. Whatever approach is taken, the empirical observations which have already been made can serve as a standard by which to judge the approach.

\section{Refining the Model Class}

The substructuring examples presented in this work showed that substructuring can offer some advantages such as reduced computational effort without significant loss of sensitivity to damage. However, aside from simulation test- 
ing, no methods exist to suggest the best possible choice for a substructuring configuration. Developing criteria for choosing the best substructuring for a given structure and a given set of modal parameters would be helpful. In order to account for the problems associated with the localization of damage in substructures which contain many elements, adaptive substructuring should also be investigated.

One improvement that has not thus far been mentioned in this work is the concept of selective updating (Farhat and Hemez 1993). In selective updating, some criteria are used to choose a subset of the available structural model parameters to analyze. The consequence of employing a method such as this is to reduce the number of parameters involved in the calculations. Also, a finer substructuring mesh can be maintained since not all of the substructure parameters would need to be updated whenever data is acquired. Having a finer mesh improves the sensitivity of the SHM method to member level damage and thus enhances the ability to localize damage. Some foreseeable problems could arise in applying the probabilistic framework developed in this work since different reference undamaged PDFs might be needed for different sets of selected parameters. However, the potential advantages do warrant some investigation in this area.

\section{Computational Issues}

The underlying computational machinery required to calculate the probability of variation involves many approximations and iterative steps which introduce error into the problem. Unlike the error due to noise in the data, this error cannot be reduced by simply taking more data. Only by improving the method of calculation can the error be mitigated. A first step would therefore be to investigate the nature and degree of variation which arises due to these computational effects. For example, while the coarse approximation for asymptotic 
expansion of the probability integrals was determined to be sufficient for the examples considered in this work, it may not be in all cases. Thus, a thorough study of how well the coarse approximation works in different scenarios should be conducted. Other computational issues which should be investigated are the improvements in approximation achievable with the fine approximation method, and the error introduced by the iterative minimization scheme.

\subsubsection{Automation}

In light of the fact that the ultimate goal of this work is to create a system for use on real structures, many operational questions need to be addressed. For example, on-line determination of the modal parameters from measured data is a fundamental requirement if the SHM system is to be fully automated. Also, criteria for setting the maximum size of the monitoring cycle window used in determining the sequence of probabilities of variation need to be established. Such criteria should consider the trade-off between the increased computation and the potentially increased ability to detect low levels of damage by having longer windows. As a final matter, the interface between the SHM analysis and the end user needs to be considered.

\subsection{Limitations of The SHM Method}

The breadth of the SHM field precludes any single method from covering all of the possible aspects of the problem. Thus, a given technique may work well at handling some parts of SHM, but not work as well with other parts. Some of the strengths of the SHM method which has been introduced are covered in Section 4.3. In the present section, some of the limitations are discussed. Most of the items mentioned are fundamental difficulties associated with any

global SHM procedure. Problems which are unique to the presented method 
are noted as such.

\section{Types of Damage}

A very basic difficulty in SHM comes from establishing what types of changes in a structure can actually be detected. One of the fundamental assumptions for performing global SHM, as mentioned in Section 1.3, is that changes in the structure will affect some measured data to a sufficient extent to be able to characterize the changes. Certain types of changes may not have such effects.

For instance, plastic deformation due to high loading conditions might be considered a type of "damage" that is of importance to detect. However, once the high loading event has passed, a plastically deformed structural member will behave elastically once again, and quite possibly not alter measurements of the dynamic response of the structure. As another example, consider a highly redundant structure. If a single structural member is damaged in some fashion, the local effect might be significant, but the global effect may not be. Unless measurements are made in the specific area of the member, the damage could go undetected. Sensing these types of damaging events is a serious challenge to a global SHM method since the measured data is not significantly effected by the damage.

\section{Model Problems}

The highly redundant structure example brings up another limitation of global model-based SHM methods: The degree to which damage can be located depends on the nature of the measured data. The number of measured DOFs and significantly expressed modes of vibration in the recorded data dictate how many model parameters can be identified without uniqueness problems. If very few model parameters can be uniquely identified, the model for the structure may not accurately reflect the true behavior. Thus, the second 
assumption made in Section 1.3 could be violated. That assumption involved treating changes in the model identified from different data sets as proxies for changes in the real structure.

The method presented in this work uses substructuring to reduce the number of parameters so that they can be uniquely identified. As shown in Chapter 3, substructuring can have the effect of smearing elemental level damage over an entire substructure, so the sensitivity to damage may be reduced. This is a limitation to the presented method which, as mentioned in Section 4.1, could be addressed through adaptive substructuring (Hjelmstad and Shin 1997) or selective parameter updating (Farhat and Hemez 1993).

Another result of using models with few parameters is that even if the existence and location of damage can be found, the degree of damage will be extremely difficult to determine. This is a fundamental limitation of global SHM methods. The best way to overcome this limitation is to use the global SHM to establish existence and possible location of damage, and apply local SHM techniques to actually find and determine the extent of the damage.

\section{Problems From Using Modal Data}

The advantages of using modal parameters in the SHM process have already been commented upon in Section 1.3. There are, however, disadvantages. The modal parameters themselves are identified using some system identification technique to fit the parameters of a modal model to measured data. Thus, uncertainties are introduced which were ascribed to "noise" in this work. The uncertainties are passed on to the model parameters identified using the modal parameters. Suppose a significant portion of the variation in the modal parameters when there is no damage is due to the identification technique and not the underlying measured data. Performing SHM using the measured time history data directly may then offer some advantages. 


\section{Fooling a SHM Method}

The final problem with SHM occurs when what appears to be damage may not be damage. Following an extreme loading event (e.g. earthquake), many concrete structures exhibit a decrease in their frequencies of vibration. This effect is believed to be caused by a combination of loosening of the concrete, the soil, and the non-structural elements in the structure. Over time, some of the frequencies may increase slightly, but they generally stay lower than the original values. Any reasonable SHM technique would interpret the change as damage and identify the possible damage locations. In reality, however, the structure may not be truly damaged. Thus, interpreting damage in concrete structures may present difficulties.

\subsection{Conclusions}

The need for a structural health monitoring which took into account the model parameter uncertainty in the problem and lent itself well to on-line application motivated this work. The results of this study represent the first step in the development of a SHM method to meet these two requirements. In order to accomplish the tasks, a model-based approach to SHM was taken.

The method presented in this work departed from traditional deterministic model-based SHM techniques by answering a different question in order to solve the problem. A traditional deterministic model-based technique essentially would solve the SHM problem by answering the question,

What is the change in the optimal stiffness parameters of a model identified using data from a reference undamaged condition and a current condition?

The answer to this question does not take the parameter uncertainty effects 
into account. This work explicitly considered the uncertainty effects by asking the probabilistic question,

Based on the available data, and acknowledging the uncertainty in the problem, what is the probability that the current model stiffness parameters are less than some reference undamaged model stiffness parameters?

In order to answer this question, probabilistic descriptions of the model stiffness parameters given different data were needed. The Bayesian framework handles these types of conditional plausibilities. Therefore, it was the ideal setting in which to pose the answer to the question.

An explicit formulation of the SHM method thus defined was presented in Chapter 2. Fundamentally, the discussion in that chapter simply developed the necessary probabilistic description for the model parameters given the data and showed how the probabilistic SHM question could be answered using those descriptions.

Once the framework was developed, testing on some simple computergenerated structure examples was presented in Chapter 3. The results of the testing showed that the measure of damage derived in Chapter 2 had some distinguishable characteristic behaviors based on whether the structure was damaged or not. Thus, it could be applied to detect damage in a structure. Some of the limitations and shortcomings of the method were also pointed out.

Based on the efforts described in this work a number of conclusions can be drawn. First, a SHM method which accounts for uncertainty in the SHM problem in a structured fashion has been successfully created. Although the method was developed to detect reductions in stiffness parameters for linear models using modal data, the conceptual framework is not limited by these constraints. If the marginal PDFs can be formulated using different model 
classes and measured data, the fundamental idea behind the method can still be applied. In such an extension of the method, there may be an increased computational cost. Also, although the probability of variation is used in this work to detect reduction in stiffness, it can be more generally applied to determine increases in the stiffness parameters as well. Such an application could arise in tracking how the properties of a concrete structure change as the concrete cures.

Another contribution of this work is the consideration of issues associated with automated application of the SHM method. While the goal of SHM should be on-line application, no examples could be found in the literature that deal with the issue. Therefore, designing the method to lend itself well to automated application and addressing some of the concerns associated with on-line monitoring are significant contributions. A considerable advantage is gained by considering the problem as one of continually monitoring a structure rather that simply performing one-shot measurement and damage detection tests. In this framework, small levels of damage can be detected by monitoring the structure over long times. This can be useful for detecting structural degradation which builds up slowly such as that from corrosion and fatigue.

Section 4.2 noted when and where the method would have trouble being applied. Fortunately, there are plenty of situations for which this SHM method is well-suited. Detecting fractures in steel and aluminum framed structures is one obvious application. Off-shore oil platforms also present a possible venue for use of this SHM method. Finally, going back to the beginning of this work, a situation like the Mianus River bridge is ideal example of where this method would have performed well. If a SHM system existed and were in place when the first key structural failure occurred, the potential danger would have been detected, and the loss of life and property would have been prevented. 


\section{Appendix A}

\section{Probability Model Details}

This appendix presents the details to some of the results presented in section 2.4 .

Proof of optimal scaling for the modeshape error

Proposition A.1 For $x, y \in \mathbb{R}^{N}$ for some $N \in \mathbb{Z}^{+}$, the value of $\eta$ which minimizes

$$
\|x-\eta y\|^{2}
$$

is

$$
\eta=\frac{\langle x, y\rangle}{\|y\|^{2}}
$$

Proof: Take the derivative of this function with respect to $\eta$, set it to zero, 
and solve for $\eta$.

$$
\begin{aligned}
\frac{\partial}{\partial \eta}\|x-\eta y\|^{2} & =\frac{\partial}{\partial \eta}\left(\|x\|^{2}-2 \eta x^{T} y+\eta^{2}\|y\|^{2}\right) \\
& =-2 x^{T} y+2 \eta y^{T} y \\
& =-2\langle x, y\rangle+2 \eta\|y\|^{2} \\
& =0
\end{aligned}
$$

This gives

$$
\eta=\frac{\langle x, y\rangle}{\|y\|^{2}}
$$

Since the second derivative of $f(x, y, \eta)$ with respect to $\eta$ is clearly positive, the $\eta$ given in equation A.1 minimizes the norm with respect to $\eta$.

\section{Algebraic Details of Various Steps}

Proposition A.2 Equation (2.25a) is true, that is,

$$
p\left(\psi_{r} \mid \theta, \alpha_{r}, \epsilon_{\psi_{r}}\right)=k_{r} \exp \left[-\frac{1}{2} \frac{1}{\epsilon_{\psi_{r}}^{2}} \sin ^{2} \beta_{r}\right]
$$


Proof: Start with (2.24) and manipulate.

$$
\begin{aligned}
p\left(\psi_{r} \mid \theta, \alpha_{r}, \epsilon_{\psi_{r}}\right) & =k_{r} \exp \left[-\frac{1}{2} \frac{1}{\epsilon_{\psi_{r}}^{2}}\left\|\frac{\psi_{r}}{\left\|\psi_{r}\right\|}-\frac{\left\langle\psi_{r}, \Gamma \phi_{r}\right\rangle}{\left\|\psi_{r}\right\|\left\|\Gamma \phi_{r}\right\|} \frac{\Gamma \phi_{r}}{\left\|\Gamma \phi_{r}\right\|}\right\|^{2}\right] \\
& =k_{r} \exp \left[-\frac{1}{2} \frac{1}{\epsilon_{\psi_{r}}^{2}}\left(1-2 \frac{\left\langle\psi_{r}, \Gamma \phi_{r}\right\rangle^{2}}{\left\|\psi_{r}\right\|^{2}\left\|\Gamma \phi_{r}\right\|^{2}}+\frac{\left\langle\psi_{r}, \Gamma \phi_{r}\right\rangle^{2}}{\left\|\psi_{r}\right\|^{2}\left\|\Gamma \phi_{r}\right\|^{2}}\right)\right] \\
& =k_{r} \exp \left[-\frac{1}{2} \frac{1}{\epsilon_{\psi_{r}}^{2}}\left(1-\frac{\left\langle\psi_{r}, \Gamma \phi_{r}\right\rangle^{2}}{\left\|\psi_{r}\right\|^{2}\left\|\Gamma \phi_{r}\right\|^{2}}\right)\right] \\
& =k_{r} \exp \left[-\frac{1}{2} \frac{1}{\epsilon_{\psi_{r}}^{2}}\left(1-\cos ^{2} \beta_{r}\right)\right] \\
& =k_{r} \exp \left[-\frac{1}{2} \frac{1}{\epsilon_{\psi_{r}}^{2}} \sin ^{2} \beta_{r}\right] .
\end{aligned}
$$

Proposition A.3 Equation 2.31 is true, that is,

$$
\begin{aligned}
p\left(\omega_{r}^{2} \mid \theta, \alpha_{r}, \sigma_{\omega_{r}^{2}}\right)=\frac{1}{f_{r}\left(\theta, \alpha_{r}, \sigma_{\omega_{r}^{2}}\right)} \exp \left[-\frac{1}{2} \frac{1}{\sigma_{\omega_{r}^{2}}^{2}}(\right. & \left(\omega_{r}^{2}-\frac{\alpha_{r}^{T} \Omega^{2} \alpha_{r}}{\alpha_{r}^{T} \alpha_{r}}\right)^{2}+ \\
& \left.\left.\frac{\left(\alpha_{r}^{T} \Omega^{4} \alpha_{r}\right)}{\alpha_{r}^{T} \alpha_{r}}-\frac{\left(\alpha_{r}^{T} \Omega^{2} \alpha_{r}\right)^{2}}{\left(\alpha_{r}^{T} \alpha_{r}\right)^{2}}\right)\right] .
\end{aligned}
$$

Proof: Expand the square term on the right side in equation (2.28) and manipulate the result. The $f_{r}$ is multiplied through, and a new function $p^{*} \equiv f_{r} p$ 
is defined in order to simplify the notation.

$$
\begin{aligned}
p^{*}\left(\omega_{r}^{2} \mid \theta, \alpha_{r}, \sigma_{\omega_{r}^{2}}\right) & =\exp \left[-\frac{1}{2} \frac{1}{\sigma_{\omega_{r}^{2}}^{2}} \frac{1}{\alpha_{r}^{T} \alpha_{r}} \sum_{s=1}^{N_{d}} \alpha_{r s}^{2}\left(\omega_{r}^{4}-2 \omega_{r}^{2} \omega_{s}^{2}(\theta)+\omega_{s}^{4}(\theta)\right)\right] \\
& =\exp \left[-\frac{1}{2} \frac{1}{\sigma_{\omega_{r}^{2}}^{2}} \frac{1}{\alpha_{r}^{T} \alpha_{r}} \alpha_{r}^{T}\left(\omega_{r}^{4} I-2 \omega_{r}^{2} \Omega^{2}(\theta)+\Omega^{4}(\theta)\right) \alpha_{r}\right] \\
& =\exp \left[-\frac{1}{2} \frac{1}{\sigma_{\omega_{r}^{2}}^{2}}\left(\omega_{r}^{4}-2 \frac{\alpha_{r}^{T} \Omega^{2} \alpha_{r}}{\alpha_{r}^{T} \alpha_{r}} \omega_{r}^{2}+\frac{\alpha_{r}^{T} \Omega^{4} \alpha_{r}}{\alpha_{r}^{T} \alpha_{r}}\right)\right] \\
& =\exp \left[-\frac{1}{2} \frac{1}{\sigma_{\omega_{r}^{2}}^{2}}\left(\left(\omega_{r}^{2}-\frac{\alpha_{r}^{T} \Omega^{2} \alpha_{r}}{\alpha_{r}^{T} \alpha_{r}}\right)^{2}+\frac{\left(\alpha_{r}^{T} \Omega^{4} \alpha_{r}\right)}{\alpha_{r}^{T} \alpha_{r}}-\frac{\left(\alpha_{r}^{T} \Omega^{2} \alpha_{r}\right)^{2}}{\left(\alpha_{r}^{T} \alpha_{r}\right)^{2}}\right)\right] .
\end{aligned}
$$

Proposition A.4 Equation 2.32 is true, that is,

$$
p\left(\omega_{r}^{2} \mid \theta, \alpha_{r}, \sigma_{\omega_{r}^{2}}\right)=\frac{1}{f_{r}\left(\theta, \alpha_{r}, \sigma_{\omega_{r}^{2}}\right)} \exp \left[-\frac{1}{2} \frac{1}{\sigma_{\omega_{r}^{2}}^{2}} \frac{1}{\left\|\phi_{r}\right\|_{M}^{2}}\left\|\left(K-\omega_{r}^{2} M\right) \phi_{r}\right\|_{M^{-1}}^{2}\right]
$$

Proof: The PDF can also be expressed in terms of $K, M$, and $\phi_{r}$. Use is made of the facts that $M$ and $K$ are symmetric. The $f_{r}$ is multiplied through, and a new function $p^{*} \equiv f_{r} p$ is defined in order to simplify the notation. Starting 
with the second line from the proof of Proposition A.3 gives

$$
\begin{aligned}
p^{*}\left(\omega_{r}^{2} \mid \theta, \alpha_{r}\right) & =\exp \left[-\frac{1}{2} \frac{1}{\sigma_{\omega_{r}^{2}}^{2}} \frac{\alpha_{r}^{T}\left(\omega_{r}^{4} I-2 \omega_{r}^{2} \Omega^{2}(\theta)+\Omega^{4}(\theta)\right) \alpha_{r}}{\alpha_{r}^{T} \alpha_{r}}\right] \\
& =\exp \left[-\frac{1}{2} \frac{1}{\sigma_{\omega_{r}^{2}}^{2}} \frac{1}{\alpha_{r}^{T} \alpha_{r}} \alpha_{r}^{T}\left(\omega^{2} I-\Omega^{2}\right)^{2} \alpha_{r}\right] \\
& =\exp \left[-\frac{1}{2} \frac{1}{\sigma_{\omega_{r}^{2}}^{2}} \frac{1}{\alpha_{r}^{T} \alpha_{r}}\left\|\left(\omega^{2} I-\Omega^{2}\right) \alpha_{r}\right\|^{2}\right] \\
& =\exp \left[-\frac{1}{2} \frac{1}{\sigma_{\omega_{r}^{2}}^{2}} \frac{1}{\alpha_{r}^{T} X^{T} M X \alpha_{r}}\left\|\left(\omega^{2} X^{T} M X-X^{T} K X\right) \alpha_{r}\right\|^{2}\right] \\
& =\exp \left[-\frac{1}{2} \frac{1}{\sigma_{\omega_{r}^{2}}^{2}} \frac{1}{\phi_{r}^{T} M \phi_{r}}\left\|X^{T}\left(\omega^{2} M-K\right) \phi_{r}\right\|^{2}\right] \\
& =\exp \left[-\frac{1}{2} \frac{1}{\sigma_{\omega_{r}^{2}}^{2}} \frac{1}{\phi_{r}^{T} M \phi_{r}} \phi_{r}^{T}\left(\omega^{2} M-K\right) X X^{T}\left(\omega^{2} M-K\right) \phi_{r}\right] \\
& =\exp \left[-\frac{1}{2} \frac{1}{\sigma_{\omega_{r}^{2}}^{2}} \frac{1}{\phi_{r}^{T} M \phi_{r}} \phi_{r}^{T}\left(\omega^{2} M-K\right) M^{-1}\left(\omega^{2} M-K\right) \phi_{r}\right] \\
& =\exp \left[-\frac{1}{2} \frac{1}{\sigma_{\omega_{r}^{2}}^{2}} \frac{1}{\left\|\phi_{r}\right\|_{M}^{2}}\left\|\left(K-\omega_{r}^{2} M\right) \phi_{r}\right\|_{M^{-1}}^{2}\right] .
\end{aligned}
$$

Proposition A.5 Equation 2.36 is true, that is,

$$
f_{r}\left(\theta, \alpha_{r}, \sigma_{\omega_{r}^{2}}\right)=\frac{1}{\sqrt{2 \pi} \sigma_{\omega_{r}^{2}}} \exp \left[-\frac{1}{2} \frac{1}{\sigma_{\omega_{r}^{2}}^{2}} \frac{1}{\left\|\phi_{r}\right\|_{M}^{2}}\left\|\left(K-\tilde{\omega}_{r}^{2} M\right) \phi_{r}\right\|_{M^{-1}}^{2}\right]
$$


Proof: Starting with (2.33) and manipulating gives

$$
\begin{aligned}
f_{r}\left(\theta, \alpha_{r}, \sigma_{\omega_{r}^{2}}\right)=\frac{1}{\sqrt{2 \pi} \sigma_{\omega_{r}^{2}}} \exp \left[\frac{1}{2} \frac{1}{\sigma_{\omega_{r}^{2}}^{2}}\left(\frac{\alpha_{r}^{T} \Omega^{4} \alpha_{r}}{\alpha_{r}^{T} \alpha_{r}}-\frac{\left(\alpha_{r}^{T} \Omega^{2} \alpha_{r}\right)^{2}}{\left(\alpha_{r}^{T} \alpha_{r}\right)^{2}}\right)\right] \\
=\frac{1}{\sqrt{2 \pi} \sigma_{\omega_{r}^{2}}} \exp \left[\frac{1}{2} \frac{1}{\sigma_{\omega_{r}^{2}}^{2}} \frac{1}{\alpha_{r}^{T} \alpha^{r}} \alpha_{r}^{T}\left(\Omega^{4}-2 \frac{\alpha_{r}^{T} \Omega^{2} \alpha_{r}}{\alpha_{r}^{T} \alpha_{r}} \Omega^{2}+\frac{\left(\alpha_{r}^{T} \Omega^{2} \alpha_{r}\right)^{2}}{\left(\alpha_{r}^{T} \alpha_{r}\right)^{2}}\right) \alpha_{r}\right] \\
=\frac{1}{\sqrt{2 \pi} \sigma_{\omega_{r}^{2}}} \exp \left[\frac{1}{2} \frac{1}{\sigma_{\omega_{r}^{2}}^{2}} \frac{1}{\alpha_{r}^{T} \alpha^{r}} \alpha_{r}^{T}\left(\Omega^{2}-\frac{\alpha_{r}^{T} \Omega^{2} \alpha_{r}}{\alpha_{r}^{T} \alpha_{r}} I\right)^{2} \alpha_{r}\right] \\
=\frac{1}{\sqrt{2 \pi} \sigma_{\omega_{r}^{2}}} \exp \left[\frac{1}{2} \frac{1}{\sigma_{\omega_{r}^{2}}^{2}} \frac{1}{\alpha_{r}^{T} X^{T} M X \alpha^{r}}\left\|\left(\Omega^{2}-\frac{\alpha_{r}^{T} \Omega^{2} \alpha_{r}}{\alpha_{r}^{T} \alpha_{r}} I\right) \alpha_{r}\right\|^{2}\right] \\
=\frac{1}{\sqrt{2 \pi} \sigma_{\omega_{r}^{2}}} \exp \left[\frac{1}{2} \frac{1}{\sigma_{\omega_{r}^{2}}^{2}} \frac{1}{\phi_{r}^{T} M \phi_{r}}\left\|X^{T}\left(K-\frac{\phi_{r}^{T} K \phi_{r}}{\phi_{r}^{T} M \phi_{r}} M\right) \phi_{r}\right\|^{2}\right] \\
=\frac{1}{\sqrt{2 \pi} \sigma_{\omega_{r}^{2}}} \exp \left[\frac{1}{2} \frac{1}{\sigma_{\omega_{r}^{2}}^{2}} \frac{1}{\left\|\phi_{r}\right\|_{M}^{2}}\left\|\left(K-\tilde{\omega}_{r}^{2} M\right) \phi_{r}\right\|_{M^{-1}}^{2}\right] .
\end{aligned}
$$




\section{Appendix B}

\section{Calculating the Minimum and Hessian}

In order to evaluate the probability of damage, $P^{\text {dam }}$, defined by $(2.83)$, the marginal distributions for the updated PDF defined by (2.72) need to be determined. Due to the difficultly of performing the integration analytically or numerically, two asymptotic expansion techniques for evaluating the necessary integrals were introduced in Section 2.5.8: the coarse and fine asymptotic expansion approximation methods. In order to perform the approximation, determining the minimum and Hessian of the measure of fit (MOF), $\tilde{J}(\theta)$, defined using (2.65), (2.66), (2.69), and (2.73), is required. This appendix provides the details of the method for finding these used in this study.

\section{B.1 Preliminary Development}

The relevant relations are repeated here for convenience. These are written explicitly as functions of $\theta$ and $\phi_{r}$ rather than $\theta$ and $\alpha_{r}$. Recall that $\phi_{r}=X \alpha_{r}$. Since this mapping between $\phi_{r}$ and $\alpha_{r}$ is full rank, writing in terms of $\phi_{r}$ rather than $\alpha_{r}$ does not introduce any problems. The vector $\phi$ refers to all of the $\phi_{r}$ stacked in a single vector. 
The modal measure of fit (MMOF) is

$J_{r}\left(\theta, \phi_{r}\right)=\sum_{n=1}^{N_{s}} s(\dot{n})\left[\frac{\left\|\left(K-\hat{\omega}_{r}^{2}(n) M\right) \phi_{r}\right\|_{M^{-1}}^{2}}{\sigma_{\omega_{r}^{2}}^{2}\left\|\phi_{r}\right\|_{M}^{2}}+\frac{\phi_{r}^{T} \Gamma^{T}\left(I-\psi_{r}(n) \psi_{r}^{T}(n)\right) \Gamma \phi_{r}}{\epsilon_{\psi_{r}}^{2}\left\|\Gamma \phi_{r}\right\|^{2}}\right]$.

The overall MOF is

$$
J(\theta, \phi)=\left(\theta-\theta_{F}\right)^{T} S^{-1}\left(\theta-\theta_{F}\right)+\sum_{n=1}^{N_{m}} J_{r}\left(\theta, \phi_{r}\right)
$$

The MOF for the marginal distribution on $\theta$ is

$$
\tilde{J}(\theta)=J(\theta, \hat{\phi}(\theta))
$$

where $\hat{\phi}_{r}$ is selected to satisfy

$$
\frac{\partial J(\theta, \phi)}{\partial \phi_{r}}=0
$$

for a fixed $\theta$.

\section{B.1.1 Alternate Forms of the MMOF}

Two more compact and convenient forms of (B.1) can be expressed. These are

$$
J_{r}\left(\theta, \phi_{r}\right)=\left[\frac{\phi_{r}^{T} Q_{1 r}(\theta) \phi_{r}}{\phi_{r}^{T} R_{1} \phi_{r}}+\frac{\phi_{r}^{T} Q_{2 r} \phi_{r}}{\phi_{r}^{T} R_{2} \phi_{r}}\right]
$$


and

$$
J_{r}\left(\theta, \phi_{r}\right)=\left(\sum_{n=1}^{N_{s}} s(n)\right) \theta^{T} A_{r}\left(\phi_{r}\right)^{T} A_{r}\left(\phi_{r}\right) \theta+2 \theta^{T} A_{r}\left(\phi_{r}\right)^{T} b_{r}\left(\phi_{r}\right)+C_{r}\left(\phi_{r}\right)
$$

where

$$
\begin{aligned}
Q_{1 r} & =\sum_{n=1}^{N_{s}}\left[\frac{s(n)}{\sigma_{\omega_{r}^{2}}^{2}}\left(K(\theta)-\hat{\omega}_{r}^{2}(n) M\right) M^{-1}\left(K(\theta)-\hat{\omega}_{r}^{2}(n) M\right)\right] \\
R_{1} & =M \\
Q_{2 r} & =\sum_{n=1}^{N_{s}}\left[\frac{s(n)}{\epsilon_{\psi_{r}}^{2}} \Gamma^{T}\left(I-\hat{\psi}_{r}(n) \hat{\psi}_{r}^{T}(n)\right) \Gamma\right] \\
R_{2} & =\Gamma^{T} \Gamma \\
A_{r}\left(\phi_{r}\right) & =\frac{1}{\sigma_{\omega_{r}^{2}}} \frac{1}{\left\|\phi_{r}\right\|_{M}}\left[M^{-\frac{1}{2}} K_{1} \phi_{r} \cdots M^{-\frac{1}{2}} K_{N_{\theta}} \phi_{r}\right]
\end{aligned}
$$

and

$$
b_{r}\left(\phi_{r}\right)=\frac{1}{\sigma_{\omega_{r}^{2}}} \sum_{n=1}^{N_{s}} s(n) \frac{1}{\left\|\phi_{r}\right\|_{M}}\left(M^{-\frac{1}{2}} K_{0}-\hat{\omega}_{r}^{2}(n) M^{\frac{1}{2}}\right) \phi_{r}
$$

The term $C_{r}\left(\phi_{r}\right)$ is never actually used in any calculations, so its form is not expressed here.

Using (B.5b), $J(\theta, \phi)$ can be expressed as

$$
J(\theta, \phi)=\left(\theta-\theta_{F}\right)^{T} S^{-1}\left(\theta-\theta_{F}\right)+\left(\sum_{n=1}^{N_{s}} s(n)\right) \theta^{T} A^{T} A \theta+2 \theta^{T} A^{T} b+C,
$$

where

$$
A=\left[\begin{array}{lll}
A_{1}^{T} & \cdots & A_{N_{m}}^{T}
\end{array}\right]^{T}
$$


and

$$
b=\left[\begin{array}{lll}
b_{1}^{T} & \cdots & b_{N_{m}}^{T}
\end{array}\right]^{T} .
$$

The term $C$ is not a function of $\theta$ and is never explicitly used, so its form is not listed.

\section{B.1.2 Derivatives of $J(\theta, \alpha)$}

Using (B.5a) and (B.5b), the partial derivatives of $J(\theta, \alpha)$ can be determined very easily.

The first partial derivative with respect to $\theta$ is

$$
\frac{\partial J}{\partial \theta}=2 S^{-1}\left(\theta-\theta_{F}\right)+2\left(\sum_{n=1}^{N_{s}} s(n)\right) A^{T} A \theta+2 A^{T} b \quad \in \mathbb{R}^{N_{\theta}} .
$$

The first partial derivative with respect to $\phi_{r}$ is

$$
\frac{\partial J}{\partial \phi_{r}}=\frac{\partial J_{r}}{\partial \phi_{r}}=2 \sum_{n=1}^{2}\left[\frac{Q_{n r} \phi_{r}}{\phi_{r}^{T} R_{n} \phi_{r}}-\frac{\phi_{r}^{T} Q_{n r} \phi_{r}}{\left(\phi_{r}^{T} R_{n} \phi_{r}\right)^{2}} R_{n} \phi_{r}\right] \in \mathbb{R}^{N_{d}}
$$

The second partial derivative with respect to $\theta$ is

$$
\frac{\partial^{2} J}{\partial \theta^{2}}=2 S^{-1}+2\left(\sum_{n=1}^{N_{s}} s(n)\right) A^{T} A \in \mathbb{R}^{N_{\theta} \times N_{\theta}}
$$

Using (B.1) directly, the second partial derivative with respect to $\theta$ can also be expressed by

$$
\left[\frac{\partial^{2} J}{\partial \theta^{2}}\right]_{i j}=2\left[S^{-1}\right]_{i j}+\sum_{r=1}^{N_{m}} \sum_{n=1}^{N_{s}}\left[\frac{s(n)}{\sigma_{\omega_{r}^{2}}^{2}} \frac{\phi_{r}\left(K_{j} M^{-1} K_{i}+K_{i} M^{-1} K_{j}\right) \phi_{r}}{\phi_{r}^{T} M \phi_{r}}\right] .
$$


The second partial derivative with respect to $\phi_{r}$ is

$$
\begin{aligned}
\frac{\partial^{2} J}{\partial \phi_{r}^{2}}= & 2 \sum_{n=1}^{2}\left[\frac{Q_{n r}}{\phi_{r}^{T} R_{n} \phi_{r}}-\frac{2}{\left(\phi_{r}^{T} R_{n} \phi_{r}\right)^{2}}\left(Q_{n r} \phi_{r} \phi_{r}^{T} R_{n}+R_{n} \phi_{r} \phi_{r}^{T} Q_{n r}\right)-\right. \\
& \left.\frac{\phi_{r}^{T} Q_{n r}(\theta) \phi_{r}}{\left(\phi_{r}^{T} R_{n} \phi_{r}\right)^{2}} R_{n}+4 \frac{\phi_{r}^{T} Q_{n r}(\theta) \phi_{r}}{\left(\phi_{r}^{T} R_{n} \phi_{r}\right)^{3}} R_{n} \phi_{r} \phi_{r}^{T} R_{n}\right] \in \mathbb{R}^{N_{d} \times N_{d}}
\end{aligned}
$$

The cross derivatives between different expanded modeshapes are all zero, so only the cross derivative between $\theta$ and $\phi_{r}$ remains. This cross derivative is

$$
\frac{\partial^{2} J}{\partial \phi_{r} \partial \theta} \in \mathbb{R}^{N_{d} \times N_{\theta}}
$$

where the $\mathrm{i}^{\text {th }}$ column is given by

$$
\left[\frac{\partial^{2} J}{\partial \phi_{r} \partial \theta}\right]_{i}=\frac{Q_{1 r, i}}{\phi_{r}^{T} R_{1} \phi_{r}} \phi_{r}-\frac{\phi_{r}^{T} Q_{1 r, i} \phi_{r}}{\left(\phi_{r}^{T} R_{1} \phi_{r}\right)^{2}} R_{1} \phi_{r} \in \mathbb{R}^{N_{d}}
$$

and

$Q_{1 r, i} \equiv \frac{\partial Q_{1 r}}{\partial \theta_{i}}=\sum_{n=1}^{N_{s}}\left[\frac{s(n)}{\sigma_{\omega_{r}^{2}}^{2}}\left(\left(K-\hat{\omega}_{r}^{2}(n) M\right) M^{-1} K_{i}+K_{i} M^{-1}\left(K-\hat{\omega}_{r}^{2}(n) M\right)\right)\right]$.

\section{B.1.3 Some Final Notation}

Some useful shorthand for the development to follow is now defined.

$$
\frac{\partial(\cdot)}{\partial \phi} \equiv\left[\begin{array}{c}
\frac{\partial(\cdot)}{\partial \phi_{1}} \\
\vdots \\
\frac{\partial(\cdot)}{\partial \phi_{N_{m}}}
\end{array}\right]
$$


Also,

$$
\nabla_{x y}(\cdot)=\left[\begin{array}{l}
\frac{\partial(\cdot)}{\partial x} \\
\frac{\partial(\cdot)}{\partial y}
\end{array}\right]
$$

With the preliminary material covered, the minimization routine and Hessian calculation may be described.

\section{B.2 Minimizing the $\mathrm{MOF}$}

The minimum of $\tilde{J}(\theta)$ must be found in order to determine the maxima of the updated PDF. The minimum finding procedure developed for this study is only a locally minimizing routine. The details of the development, use, and extensive testing of the minimizing method are not presented in this study since the focus is on the SHM application. Also, any algorithm which finds the minimum of the MOF could be used for finding the minimum, so the choice of a routine is not critical to the SHM.

At the minimum of $\tilde{J}(\theta)$,

$$
\frac{d \tilde{J}}{d \theta}=0 .
$$

Since $\tilde{J}(\theta)=J(\theta, \hat{\phi}(\theta))$,

$$
\begin{aligned}
& \frac{d \tilde{J}}{d \theta}=\frac{\partial J}{\partial \theta}+\sum_{r=1}^{N_{m}}\left[\left(\frac{\partial \phi_{r}}{\partial \theta}\right)^{T} \frac{\partial J}{\partial \phi_{r}}\right]
\end{aligned}
$$

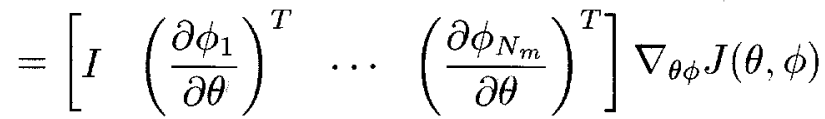

$$
\begin{aligned}
& =W(\theta, \hat{\phi}) \nabla_{\theta \phi} J(\theta, \phi) \text {. }
\end{aligned}
$$

Recall that $\hat{\phi}(\theta)$ is defined such that (B.4) holds. Thus, the minimum of $\tilde{J}(\theta)$ 
is found by simultaneously solving

$$
\begin{aligned}
& \frac{\partial J}{\partial \theta}=0 \\
& \frac{\partial J}{\partial \phi_{r}}=0 \quad r \in 1, \ldots, N_{m} .
\end{aligned}
$$

Solving (B.25) and (B.26) simultaneously is not a simple problem since there are nonlinear cross terms. However, looking at (B.13) and (B.14) reveals that solving $\frac{\partial J}{\partial \theta}=0$ with $\phi$ fixed and $\frac{\partial J}{\partial \phi_{r}}=0$ with $\theta$ fixed are fairly easy problems.

This fact suggests using an iterative procedure to find the minimum of $J$, given some initial starting point. The procedure derived from this idea can be described as follows.

- Start with an initial set of structural model parameters, $\theta_{0}$.

- Find the expanded modeshape for which $\frac{\partial J}{\partial \phi_{r}}=0$ while holding $\theta$ fixed at $\theta_{0}$.

- Fix the expanded modeshapes at these values and find $\theta$ such that $\frac{\partial J}{\partial \theta}=$ 0 .

- Iterate until a degree of convergence is achieved.

This process is capable of finding only local minima. Thus, the choice of $\theta_{0}$ will affect the solution if there is not a global minima of $J$. This is not a problem for the application in this study since the model classes were all assumed to be globally identifiable.

Unfortunately, no proof has been devised which shows that this method will actually converge on the local minimum. The only guarantee is that during each minimization, the MOF decreases. Extensive use of the method (Beck and Vanik 1996) for cases where the minimum was known has indicated that 
it does approach the local minimum in a very rapid fashion. A possible course of future study might be to investigate how well this minimization method performs against others that are suited to this problem.

\section{Minimizing With Respect to $\theta$}

In order to minimize $J$ with respect $\theta$, the $\theta$ is found so that $\frac{\partial J}{\partial \theta}=0$. Recall from (B.13) that

$$
\frac{\partial J}{\partial \theta}=2 S^{-1}\left(\theta-\theta_{F}\right)+2 A^{T} A \theta+2 A^{T} b
$$

Using (B.13) in (B.25) and solving for $\theta$ gives

$$
\theta=-\left(S^{-1}+A^{T} A\right)^{-1}\left(A^{T} b-S^{-1} \theta_{F}\right)
$$

The term $S^{-1}+A^{T} A$ is always invertible since $S$ is positive definite. There might be ill-conditioning problems if $A^{T} A$ is ill-conditioned. If $A^{T} A$ is illconditioned, the implication is that an insufficient number of observable parameter are available to identify the number of model parameters. A possible future course of study is to see if the reducing the ill-conditioning of $A^{T} A$ for a given amount of modal data can be used to set the number of structural model parameters and determine optimal substructuring.

\section{Minimizing With Respect to $\phi$}

In this section, only minimization with respect to a single $\phi_{r}$ is considered, since there is no dependence on coupled expanded modeshape vectors in the MOF. The first derivative of $J$ with respect to $\phi_{r}$ is (B.14)

$$
\frac{\partial J}{\partial \phi_{r}}=\frac{\partial J_{r}\left(\theta, \phi_{r}\right)}{\partial \phi_{r}}=2 \sum_{n=1}^{2}\left[\frac{Q_{n r} \phi_{r}}{\phi_{r}^{T} R_{n} \phi_{r}}-\frac{\phi_{r}^{T} Q_{n r} \phi_{r}}{\left(\phi_{r}^{T} R_{n} \phi_{r}\right)^{2}} R_{n} \phi_{r}\right] \in \mathbb{R}^{N_{d}}
$$


By adding and subtracting the term

$$
2 \frac{\phi_{r}^{T} Q_{2 r} \phi_{r}}{\left(\phi_{r}^{T} R_{2} \phi_{r}\right)^{2}} \frac{R_{1} \phi_{r}}{\left(\phi_{r}^{T} R_{1} \phi_{r}\right)^{2}}
$$

the derivative can be rewritten as

$$
\begin{aligned}
& \text { (B.30) } \frac{\partial J}{\partial \phi_{r}}=\left[2 \frac{Q_{1 r}}{\phi_{r}^{T} R_{1} \phi_{r}}+2 \frac{Q_{2 r}}{\phi_{r}^{T} R_{2} \phi_{r}}-2 \frac{\phi_{r}^{T} Q_{2 r} \phi_{r}}{\left(\phi_{r}^{T} R_{2} \phi_{r}\right)^{2}} R_{1}+\right. \\
& \left.2 \frac{\phi_{r}^{T} Q_{2 r} \phi_{r}}{\left(\phi_{r}^{T} R_{2} \phi_{r}\right)^{2}} \frac{R_{1}}{\left(\phi_{r}^{T} R_{1} \phi_{r}\right)^{2}}-2\left(\frac{\phi_{r}^{T} Q_{1 r} \phi_{r}}{\left(\phi_{r}^{T} R_{1} \phi_{r}\right)^{2}} \frac{1}{\left(\phi_{r}^{T} R_{2} \phi_{r}\right)^{2}}+2 \frac{\phi_{r}^{T} Q_{2 r} \phi_{r}}{\left(\phi_{r}^{T} R_{2} \phi_{r}\right)^{2}}\right) R_{1}\right] \phi_{r}=0 .
\end{aligned}
$$

Summing the last two terms in the expression above gives $J_{r}$ multiplied by $-2 R_{1}$. After incorporating the normalization constraint on $\phi_{r}$, the expression can be written as

$$
\frac{\partial J}{\partial \phi_{r}}=\left[U_{r}\left(\theta, \phi_{r}\right)-2 J_{r}\left(\theta, \phi_{r}\right) R_{1}\right] \phi_{r}=0
$$

where

$$
U_{r}\left(\theta, \phi_{r}\right)=2 Q_{1 r}+2 \frac{Q_{2 r}}{\phi_{r}^{T} R_{2} \phi_{r}}+2 \frac{\phi_{r}^{T} Q_{2 r} \phi_{r}}{\phi_{r}^{T} R_{2} \phi_{r}}\left(R_{1}-\frac{1}{\phi_{r}^{T} R_{2} \phi_{r}} R_{2}\right)
$$

If $U_{r}\left(\theta, \phi_{r}\right)$ were not a function of $\phi_{r}$, the solution would be simple, since this would be a generalized eigenvalue problem. In order to minimize $J$, the eigenvector corresponding to the smallest eigenvalue would be found. However, $U_{r}\left(\theta, \phi_{r}\right)$ is a function of $\phi_{r}$. Therefore, an iterative approach is taken to solving the problem. A seed value, $\phi_{r 0}$, is chosen and $\phi_{r 1}$ calculated using $U_{r}\left(\theta, \phi_{r 0}\right)$. The $\phi_{r}$ are iteratively calculated until a subjectively set convergence criteria is met.

As with the minimization iterating scheme, this one is not guaranteed to converge to a fixed point. However, experience with the method has shown 
that it tends to converge to a proper solution. A more rigorous approach to proving that this method converges is another topic for future study.

\section{B.3 Derive the Hessian}

The Hessian of the MOF can be calculated by taking the derivative of (B.24):

$$
\begin{gathered}
L_{\theta}=\frac{d^{2} \tilde{J}}{d \theta^{2}}=\frac{\partial^{2} J}{\partial \theta^{2}}+\sum_{r=1}^{N_{m}}\left[\left(\frac{\partial \phi_{r}}{\partial \theta}\right)^{T} \frac{\partial^{2} J}{\partial \phi_{r} \partial \theta}+\left(\frac{\partial^{2} J}{\partial \phi_{r} \partial \theta}\right)^{T} \frac{\partial \phi_{r}}{\partial \theta}+\right. \\
\left.\left(\frac{\partial \phi_{r}}{\partial \theta}\right)^{T} \frac{\partial^{2} J}{\partial \phi_{r}^{2}}\left(\frac{\partial \phi_{r}}{\partial \theta}\right)^{T}\right]+F\left(\nabla_{\theta \phi} J\right) .
\end{gathered}
$$

At the minimum of $\tilde{J}, F\left(\nabla_{\theta \phi} J\right)=0$, and the remaining term can be written as

$$
L_{\theta}(\hat{\theta})=W(\theta, \hat{\phi})\left[\begin{array}{cccc}
\frac{\partial^{2} J}{\partial \theta^{2}} & \left(\frac{\partial^{2} J}{\partial \phi_{1} \partial \theta}\right)^{T} & \cdots & \left(\frac{\partial^{2} J}{\partial \phi_{N_{m}} \partial \theta}\right)^{T} \\
\frac{\partial^{2} J}{\partial \phi_{1} \partial \theta} & \frac{\partial^{2} J}{\partial \phi_{1}^{2}} & & 0 \\
\vdots & & \ddots & \\
\frac{\partial^{2} J}{\partial \phi_{N_{m}} \partial \theta} & 0 & & \frac{\partial^{2} J}{\partial \phi_{N_{m}}^{2}}
\end{array}\right] W^{T}(\theta, \hat{\phi}) .
$$

All of the terms in (B.34) except for $\frac{d \phi_{r}}{d \theta}$ can be calculated analytically using (B.15), (B.17), and (B.19).

Terms of the form $\frac{d \phi_{r}}{d \theta}$ are found using (B.4). Since (B.4) is true for any $\theta$, the derivative of (B.4) with respect to $\theta$ can be taken, giving

$$
\left(\frac{\partial^{2} J}{\partial \phi_{r} \partial \theta}\right)^{T}+\left(\frac{\partial^{2} J}{\partial \phi_{r}^{2}}\right) \frac{d \phi_{r}}{d \theta}=0
$$

The system of equations is solved for $\frac{d \phi_{r}}{d \theta}$ using the singular value decompo- 
sition. This procedure for finding $\frac{d \phi_{r}}{d \theta}$ is simply an application of the implicit function theorem (Rudin 1976). 


\section{Bibliography}

Agbabian, M. S. and S. F. Masri (Eds.) (1988, February). Proceedings of the International Workshop on Nondestructive Evaluation for Performance of Civil Structures, Los Angeles, CA. NSF: Department of Civil Engineering, University of Southern California.

Applied Technology Council (1989). Procedures for Postearthquake Safety Evaluation of Buildings (ATC-20). Redwood City, CA: Applied Technology Council.

Baruh, H. and S. Ratan (1993). Damage detection in flexible structures. Journal of Sound and Vibration 166(1), 21-30.

Beck, J. L. (1996). System identification methods applied to measured seismic response. In Proceedings Eleventh World Conference on Earthquake Engineering, Acapulco, Mexico.

Beck, J. L. and L. S. Katafygiotis (1992). Probabilistic system identification and health monitoring of structures. In Proceedings Tenth World Conference on Earthquake Engineering, Madrid, Spain.

Beck, J. L., B. S. May, and D. C. Polidori (1994). Determination of modal parameters from ambient vibration data for structural health monitoring. In Proceedings First World Conference on Structural Control, Pasadena, California, pp. TA3:3-12.

Beck, J. L., B. S. May, D. C. Polidori, and M. W. Vanik (1995). Ambient 
vibration surveys of three steel-frame buildings strongly shaken by the 1994 Northridge earthquake. Technical Report SAC-95-04, SAC Joint Venture.

Beck, J. L. and M. W. Vanik (1996, May). Structural model updating using expanded modeshapes. In Proceedings of the 11th Engineering Mechanics Conference, Fort Lauderdale, Florida.

Beck, J. L., M. W. Vanik, and L. S. Katafygiotis (1994). Determination of stiffness changes from modal parameter changes for structural health monitoring. In Proceedings First World Conference on Structural Control, Pasadena, California, pp. TA3:13-22.

Beck, R. (1991). Fundamental problems in the application of structural identification procedures to damage detection. Technical Report EERL Report No. 91-03, California Institute of Technology.

Bleistein, N. and R. Handelsman (1986). Asymptotic Expansions for Integrals. New York, NY: Dover.

Box, G. E. P. and G. C. Tiao (1992). Bayesian Inference in Statistical Analysis. New York, New York: John Wiley and Sons, Inc.

Burdekin, F. M. (1993, February). Nondestructive testing of welded structural steelwork. Proceedings of the Institution of Civil Engineers Structures and Buildings 99(1), 89-95.

Caughey, T. K. and M. E. J. O'Kelly (1965). Classical normal modes in damped linear dynamic systems. Journal of Applied Mechanics 32(3), 583-588.

Ceravolo, R. and A. Destefano (1995, July). Damage location in structures through a connectivistic use of fem modal analyses. The International Journal Of Analytical And Experimental Modal Analysis 10(3), 178-186. 
Chen, X., F. Ansari, and H. Ding (1996, May). Embedded fiber optic displacement sensor for concrete elements. In Proceedings of the $11^{\text {th }}$ Engineering Mechanics Conference, Volume 1, New York, NY, pp. 359-365. ASCE: ASCE.

Claus, R. O. (Ed.) (1991). Proceedings of the Conference on Optical Fiber Sensor-Based Smart Materials and Structures : April 3-4, 1991, Lancaster, PA. Technomic Publishing Company.

Claus, R. O. (Ed.) (1993, February). Smart structures and materials '93: smart sensing, processing, and instrumentation, 1-4 February 1993, Bellingham, WA. The International Society for Optical Engineering.

Malhotra, V. M. and N. J. Carino (Eds.) (1991). CRC Handbook on Nondestructive Testing of Concrete. Boston: CRC.

deVries, M. J., S. H. Poland, J. L. Grace, V. Arya, K. A. Murphy, and R. O. Claus (1996). Optical fiber sensors for advanced civil structures. In Proceedings of the 11 $1^{\text {th }}$ Engineering Mechanics Conference, pp. 64-67.

EERI (1996). Supplement C to Volume 11, January 1996 Northridge Earthquake Renonnaissance Report. In W. T. Holmes and P. Somers (Eds.), Earthquake Spectra, Volume 2. Oakland, CA: EERI.

Fan, N. Y., S. Huang, A. T. Alavie, and R. M. Measures (1995, September). Rare-earth-doped fiber for structural damage assessment. Smart Materials \& Structures 4(3), 179-185.

Fares, N. and R. Maloof (1997). A probabilistic framework for detecting and identifying anomalies. Probabilistic Engineering Mechanics 12(2), 63-73.

Farhat, C. and F. M. Hemez (1993). Updating finite element dynamics models using an element-by-element sensitivity methodology. AIAA Journal 31(9), 1702-1711. 
Gerardi, J. and G. Hickman (1991). Health monitoring system for aircraft. In Proceedings of the Conference on Active Materials and Adaptive Structures, pp. 403-406. ADPA/AIAA/ASME/SPIE: IOP Publishing Ltd.

Hearn, G. and R. B. Testa (1991). Modal analysis for damage detection in structures. Journal of Structural Engineering 117(10), 3042-3063.

Hemez, F. M. and C. Farhat (1995). Structural damage detection via a finite element model updating methodology. The International Journal of Analytical and Experimental Modal Analysis 10(3), 152-166.

Hjelmstad, K. D. and S. Shin (1997). Damage detection and assessment of structures from static response. To appear in: Journal of Engineering Mechanics.

IMAC (1997). Proceedings of the 15th International Modal Analysis Conference. Union College, Schenectedy, New York: IMAC.

James, G. H., T. G. Carne, and J. P. Lauffer (1992). The natural excitation technique for modal parameter extraction from operating wind turbines. Technical Report SAND92-1666 • UC-261, Sandia National Laboratories.

Jaynes, E. T. (1978). Where do we stand on maximum entropy? In R. D. Levine and M. Tribus (Eds.), The Maximum Entropy Formalism. Cambridge, MA: MIT Press.

Jeffreys, S. H. (1939). Theory of Probability. Oxford: Clarendon Press.

Jenks, W. G., S. S. H. Sadeghi, and J. P. Wikswo (1997, February). Squids for nondestructive evaluation. Journal Of Physics D Applied Physics 30(3), 293-323.

Juang, J.-N. and R. S. Pappa (1985). An eigensystem realization algorithm for modal parameter identification and model reduction. Journal of Guidance, Control and Dynamics 8(5), 620-627. 
Kim, H. M. and T. J. Bartkowicz (1993, June). Damage detection and health monitoring of large space structures. Sound and Vibration 27(6), 12-17.

Kim, H. M., T. J. Bartkowicz, S. W. Smith, and D. Zimmerman (1995, April). Health monitoring of large structures. Sound and Vibration 29(4), 18-21.

Kudva, J. N., N. Munir, and P. Tan (1991). Damage detection in smart structures using neural networks and finite element analysis. In Proceedings of the Conference on Active Materials and Adaptive Structures, pp. 559-562. ADPA/AIAA/ASME/SPIE: IOP Publishing Ltd.

Leung, C. K. Y. and N. Elvin (1996). Micromechanics based design of optical fiber crack sensor. In Proceedings of the $11^{\text {th }}$ Engineering Mechanics Conference, Volume 1, pp. 236-239. ASCE.

Levine-West, M. B. and M. Milman (1994, August). Mode shape expansion techniques for prediction. In Proceedings 1st World Conference On Structural Control, Pasadena, California, pp. TA3:33-42.

Levine-West, M. B., M. H. Milman, and A. Kissil (1996). Mode shape expansion techniques for prediction - experimental evaluation. AIAA Journal $34(4), 821-829$.

Levy, M. and M. Salvadori (1992). Why Buildings Fall Down. New York, NY: W. W. Norton and Company.

Lyöri, V., P. Suopajärvi, S. Nissilä, H. Kopola, and H. Suni (1994). Measurements of stress in a road structure using optical fibre sensors. In Proceedings of the First World Conference on Structural Control, Pasadena, California, pp. WA3:40-48.

Madsen, H. O., S. Krenk, and N. C. Lind (1986). Methods of Structural Safety. New Jersey: Prentice-Hall. 
Mangal, L., V. G. Idichandy, and C. Ganapathy (1996, April-June). Artbased multiple neural networks for monitoring offshore platforms. Applied Ocean Research 18(2-3), 137-143.

Masri, S. F. (1997). Structural control: Past present and future. To appear in: Journal of Engineering Mechanics.

Masri, S. F., M. S. Agbabian, A. M. Abdelghaffar, M. Higazy, and R. O. Claus (1994, August). Experimental study of embedded fiberoptic straingauges in concrete structures. Journal of Engineering Mechanics 120(8), $1696-1717$.

Mazurek, D. F. and J. T. DeWolf (1990). Experimental study of bridge monitoring technique. Journal of Structural Engineering 116(9), 25322549 .

Measures, R. M. (1996, May). Structural sensing with fiber optic systems. In Proceedings of the $11^{\text {th }}$ Engineering Mechanics Conference, Volume 1, New York, NY, pp. 224-227. ASCE: ASCE.

Measures, R. M., T. Alavie, R. Maaskant, S. Karr, S. Huang, L. Grant, A. Guha-Thakurta, G. Tadros, and S. Rizkalla (1994). Fiber optic sensing of a carbon fiber prestressed concrete highway bridge. In Proceedings of the First World Conference on Structural Control, Pasadena, California, pp. TA1:31-40.

Mottershead, J. E. and M. I. Friswell (1993). Model updating in structural dynamics: A survey. Journal of Sound and Vibration 167(2), 347-375.

Natke, G. H. and J. T. P. Yao (Eds.) (1988). Proceedings of the Workshop on Structural Safety Evaluation Based on System Identification Approaches, Wiesbaden. Vieweg and Sons.

Natke, H. G. and J. T. P. Yao (1993). Model-based damage detection, localization and assessment of structures: The extended system identification 
methodology. Applied Math and Computer Science 3(3), 519-531.

O'Leary, P. N., D. A. Bagdasarian, and J. T. DeWolf (1992, May). Bridge condition assessment using signatures. In Proceedings of Nondestructive Evaluation of Civil Structures and Materials, University of Colorado, Boulder, Colorado.

Ott, J. and R. G. O'lone (1988, May 9). 737 fuselage separation spurs review of safeguards. Aviation Week and Space Technology, 92-95.

Pandey, A. K. and M. Biswas (1994). Damage detection in structures using changes in flexibility. Journal of Sound and Vibration 169(1), 3-17.

Pandey, A. K. and M. Biswas (1995). Experimental verification of flexibility difference method for locating damage in structures. Journal of Sound and Vibration 184(2), 311-328.

Papadimitriou, C., J. L. Beck, and L. Katafygiotis (1995). Asymptotic expansions for reliabilities and moments of uncertain dynamic systems. Technical Report EERL Report No. 95-05, California Institute of Technology.

Popovics, J. S. and J. L. Rose (1994, January). A survey of developments in ultrasonic NDE of concrete. IEEE Transactions On Ultrasonics Ferroelectrics And Frequency Control 41(1), 140-143.

Rubin, S. (1980, June). Ambient vibration survey of offshore platform. Journal of The Engineering Mechanics Division-ASCE 106(EM3), 425-441.

Rudin, W. (1976). Priciples of Mathematical Analysis. McGraw-Hill.

Topole, K. G. and N. Stubbs (1995, April). Non-destructive damage evaluation in complex structures from a minimum of modal parameters. Modal Analysis: The International Journal of Analytical and Experimental Modal Analysis 10(2), 95-103. 
Udd, E. (Ed.) (1991). Fiber Optic Sensors: An Introduction for Engineers and Scientists. New York, NY: Wiley.

Vold, H. and G. F. Rocklin (1982). The numerical implementation of a multi-input modal estimation method for minicomputers. In Proceedings First International Modal Analysis Conference, pp. 542-548.

Yao, J. T. P. and H. G. Natke (1994). Damage detection and reliability evaluation of existing structures. Structural Safety 15, 3-16.

Zalameda, J. N., G. L. Farley, and B. T. Smith (1994, April). A field deployable nondestructive impact damage assessment methodology for composite structures. Journal Of Composites Technology \& Research 16(2), 161-169.

Zimmerman, D. C., S. W. Smith, H. M. Kim, and T. J. Bartkowicz (1996, October). An experimental-study of structural health monitoring using incomplete measurements. Journal Of Vibration And Acoustics Transactions Of The ASME 118(4), 543-550. 